\title{
Interplay of Hydrogen Bonds in Assembling (4,4)-coordination Networks: Transformations From Open to Interpenetrated Networks via Anion Exchange**
}

\author{
Supporting information
}

Syntheses of complexes $\mathbf{2 - 4}$;

Tables of elemental analyses for 2-4 and for guest exchange reactions with 2;

IR Spectra of 1, complexes 2-4, 2 (after guest removal) and for guest exchange reactions with 2;

Table comparing the elemental analyses and IR spectra for guest exchange reactions with $\mathbf{2}$;

TG Analyses of complexes $\mathbf{2}$ and $\mathbf{3}$;

Powder XRD Patterns of guest exchange reactions with $\mathbf{2}$

Powder XRD patterns for $\mathbf{2}$ after guest removal and readsorption.

Crystallographic tables for $\mathbf{2}, \mathbf{3}$ and $\mathbf{4}$

[*] M. Sarkar and Dr. K. Biradha

Department of Chemistry

Indian Institute of Technology, Kharagpur, West Bengal, India.

Fax:+91-3222-282252; E-mail: kbiradha@chem.iitkgp.ernet.in

${ }^{\left.{ }^{* *}\right]}$ We acknowledge financial support from Department of Science and Technology and DST-Fist for single crystal X-ray facility. MS thanks CSIR for research fellowship. 


\section{Experimental Section:}

\section{Synthesis of Complex 2:}

Ethanolic solution $(20 \mathrm{~mL})$ of $\mathrm{Cu}\left(\mathrm{ClO}_{4}\right)_{2}(370.15 \mathrm{mg}, 1.0 \mathrm{mmol})$ was added to a stirred solution of the ligand $1 \mathrm{a}(596.00 \mathrm{mg}, 2.0 \mathrm{mmol})$ in $30 \mathrm{~mL}$ of EtOH. This resulted in the formation of a blue precipitate. To this solution, $20 \mathrm{~mL}$ of nitrobenzene was added. After 15-20 min. of stirring, $20 \mathrm{~mL}$ of water was added. The blue precipitate became soluble and a clear solution was obtained. This solution was filtered and kept for slow evaporation. Blue colored crystals were formed after 1 week in $70 \%$ yield.

Elemental Analysis: $\mathrm{C}_{50} \mathrm{H}_{55} \mathrm{Cl}_{2} \mathrm{Cu}_{1} \mathrm{~N}_{11} \mathrm{O}_{20}$ (1263.5): Calculated: $\mathrm{C} 47.48 \%, \mathrm{H} 4.35 \%, \mathrm{~N} 12.18 \%$; Found: C $47.34 \%$, H $4.11 \%$, N $11.83 \%$; IR (cm ${ }^{-1}$ ): 3468.5 (O-H stretch.), 3273 (N-H Stretch), 3079.47 (aromatic C-H stretch.), 1648.98 (amide C=O stretch), 1558 (amide II), 1527 (asym. N-O stretch), 1346 (sym. N-O).

\section{Synthesis of Complex 3:}

Ethanolic solution $(20 \mathrm{~mL})$ of $\mathrm{Cu}\left(\mathrm{ClO}_{4}\right)_{2}(370.15 \mathrm{mg}, 1.0 \mathrm{mmol})$ was added to a stirred solution of the ligand 1a (596.00 mg, $2.0 \mathrm{mmol})$ in $30 \mathrm{~mL}$ of EtOH. This resulted in the formation of a blue precipitate. After 15-20 min. of stirring, $20 \mathrm{~mL}$ of water was added to the solution. The blue precipitate became soluble and a clear solution was obtained. This solution was filtered and kept for slow evaporation. Blue colored crystals were formed after 7-10 days in $65 \%$ yield.

Elemental Analysis: $\mathrm{C}_{34} \mathrm{H}_{48} \mathrm{Cl}_{2} \mathrm{Cu}_{1} \mathrm{~N}_{8} \mathrm{O}_{16}$ (958.5): Calculated: $\mathrm{C} 42.56 \%$, H $5.00 \%$, N $11.68 \%$; Found: C $42.49 \%, \mathrm{H} 4.93 \%$, N $11.05 \%$.

\section{Synthesis of Complex 4:}

The crystals of the complex $3(89.45 \mathrm{mg}, 0.1 \mathrm{mmol})$ were dissolved in (1:1) water-ethanol solution $(6 \mathrm{~mL})$. To this solution, ethanolic solution $(2 \mathrm{~mL})$ of $\mathrm{NH}_{4} \mathrm{PF}_{6}(65.2 \mathrm{mg}, 0.4 \mathrm{mmol})$ was added. The resulted solution was filtered and kept for slow evaporation. Blue colored crystals were formed within a day in $90 \%$ yield.

Elemental analysis: $\mathrm{C}_{32} \mathrm{H}_{40} \mathrm{Cu}_{1} \mathrm{~F}_{12} \mathrm{~N}_{8} \mathrm{O}_{6} \mathrm{P}_{2}$ (985.18): Calculated: C 38.97\%, H 4.06\%, N 11.36\%; Found: C 39.01 $\%, \mathrm{H} 4.40 \%$, N $10.92 \%$.

\section{Guest Exchange Reactions of 2:}

The guest exchanged reactions were performed by immersing the crystals of 2 in various aromatic solvents. After 15-20 days, the exchanged crystals were characterized by elemental analysis, IR and powder XRD. Guest Exchange of $\mathbf{2}$ with Anisole: 
Elemental Analysis: $\left(\mathrm{C}_{16} \mathrm{H}_{18} \mathrm{~N}_{4} \mathrm{O}_{2}\right)_{2} \mathrm{Cu}_{1}\left(\mathrm{H}_{2} \mathrm{O}\right)_{2}\left(\mathrm{ClO}_{4}\right)_{2} .2\left(\mathrm{C}_{6} \mathrm{H}_{5} \mathrm{OCH}_{3}\right)$, (1110.5): Calculated: C 49.70\%, H 5.04\%, N 10.08\%; Found: C $49.59 \%$, H $4.97 \%$, N 9.90\%. IR: 3455 (O-H stretch), $3360 \mathrm{~cm}^{-1}$ (N-H stretch), $3062 \mathrm{~cm}^{-}$ ${ }^{1}$ (aromatic C-H stretch), $1661 \mathrm{~cm}^{-1}$ (amide $\mathrm{C}=\mathrm{O}$ ), $1549 \mathrm{~cm}^{-1}$ (amide II)

Guest Exchange of 2 with p-xylene:

Elemental Analysis: $\left(\mathrm{C}_{16} \mathrm{H}_{18} \mathrm{~N}_{4} \mathrm{O}_{2}\right)_{2} \mathrm{Cu}_{1}\left(\mathrm{H}_{2} \mathrm{O}\right)_{2}\left(\mathrm{ClO}_{4}\right)_{2} \cdot 1.5\left(\mathrm{C}_{8} \mathrm{H}_{11}\right)$, (2108.8): Calculated: C 50.1\%, H 5.22\%, N 10.63\%; Found: C $49.58 \%$, H $5.01 \%$, N 10.48\%. IR: 3455 (O-H stretch), $3360 \mathrm{~cm}^{-1}$ (N-H stretch), $3105 \mathrm{~cm}^{-}$ ${ }^{1}$ (aromatic C-H stretch), $1648 \mathrm{~cm}^{-1}$ (amide C=O), $1545 \mathrm{~cm}^{-1}$ (amide II)

Guest Exchange of 2 with Chlorobenzene:

Elemental Analysis: $\left(\mathrm{C}_{16} \mathrm{H}_{18} \mathrm{~N}_{4} \mathrm{O}_{2}\right)_{2} \mathrm{Cu}_{1}\left(\mathrm{H}_{2} \mathrm{O}\right)_{2}\left(\mathrm{ClO}_{4}\right)_{2} .1\left(\mathrm{C}_{6} \mathrm{H}_{5} \mathrm{Cl}\right)$, (1007.7): Calculated: $\mathrm{C} 45.29 \%, \mathrm{H} 4.50 \%, \mathrm{~N}$ $11.12 \%$; Found: C $45.83 \%, \mathrm{H} 4.17 \%, \mathrm{~N} 11.51 \%$.

Guest Exchange of $\mathbf{2}$ with benzonitrile:

Elemental Analysis:

Found: C 46.69\%, H 4.02\%, N $11.42 \%$.

Elemental Analysis of Complexes 2-6 and Guest Exchange Reactions with 2:

\begin{tabular}{|c|c|c|c|c|}
\hline Complex & Molecular Formula & $\begin{array}{l}\text { Molecular } \\
\text { Weight }\end{array}$ & Calculated & Experimental \\
\hline 2 & $\mathrm{C}_{50} \mathrm{H}_{55} \mathrm{Cl}_{2} \mathrm{Cu}_{1} \mathrm{~N}_{11} \mathrm{O}_{20}$ & 1263.5 & $\begin{array}{l}\text { C } 47.48 \% \text {, } \\
\text { H } 4.35 \% \text { N } 12.18 \%\end{array}$ & $\begin{array}{l}\text { C } 47.34 \%, \\
\text { H } 4.11 \%, \\
\text { N } 11.83 \%\end{array}$ \\
\hline 3 & $\mathrm{C}_{34} \mathrm{H}_{48} \mathrm{Cl}_{2} \mathrm{Cu}_{1} \mathrm{~N}_{8} \mathrm{O}_{16}$ & 958.5 & $\begin{array}{l}\text { C } 42.56 \% \text {, } \\
\text { H } 5.00 \% \text {, } 11.68 \% \\
\text { N }\end{array}$ & $\begin{array}{l}\text { C } 42.49 \% \text {, } \\
\text { H } 4.93 \% \text {, } \\
\text { N } 1.05 \%\end{array}$ \\
\hline 4 & $\mathrm{C}_{32} \mathrm{H}_{40} \mathrm{Cu}_{1} \mathrm{~F}_{12} \mathrm{~N}_{8} \mathrm{O}_{6} \mathrm{P}_{2}$ & 985.18 & $\begin{array}{l}\text { C } 38.97 \% \text {, } \\
\text { H } 4.06 \% \text {, } 11.36 \%\end{array}$ & $\begin{array}{l}\text { C } 39.01 \% \text {, } \\
\text { H } 4.40 \% \text {, } \\
\text { N } 10.92 \%\end{array}$ \\
\hline
\end{tabular}


Guest Exchange reactions of 2:

\begin{tabular}{|c|c|c|c|c|}
\hline Complex & Molecular Formula & $\begin{array}{l}\text { Mol. } \\
\text { Wt. }\end{array}$ & Calculated & Experimental \\
\hline $\begin{array}{l}\mathbf{2} \text { after immersing } \\
\text { in anisole }\end{array}$ & $\mathrm{CuL}_{2}\left(\mathrm{H}_{2} \mathrm{O}\right)_{2}(\text { Anisole })_{2}\left(\mathrm{ClO}_{4}\right)_{2}$ & 1110.5 & $\begin{array}{l}\text { C } 49.7 \% \text {, } \\
\text { H } 5.04 \% \text {, } \\
\text { N } 10.08 \%\end{array}$ & $\begin{array}{l}\text { C } 49.59 \% \text {, } \\
\text { H } 4.97 \% \text {, } \\
\text { N } 9.9 \%\end{array}$ \\
\hline $\begin{array}{l}2 \text { after immersing } \\
\text { in p-xylene }\end{array}$ & $\mathrm{CuL}_{2}\left(\mathrm{H}_{2} \mathrm{O}\right)_{2}(\text { xylene })_{1.5}\left(\mathrm{ClO}_{4}\right)$ & 2108.8 & $\begin{array}{l}\text { C } 50.1 \% \text {, } \\
\text { H } 5.22 \% \text {, } \\
\text { N } 10.63 \%\end{array}$ & $\begin{array}{l}\text { C } 49.58 \% \text {, } \\
\text { H } 5.01 \% \text {, } \\
\text { N } 10.48 \%\end{array}$ \\
\hline $\begin{array}{l}2 \text { after immersing } \\
\text { in chlorobenzene }\end{array}$ & $\begin{array}{l}\left.\mathrm{CuL}_{2}\left(\mathrm{H}_{2} \mathrm{O}\right)_{2} \text { (chlorobenzene }\right)_{1} \\
\left(\mathrm{ClO}_{4}\right)_{2}\end{array}$ & 1007.7 & $\begin{array}{l}\text { C } 45.29 \% \text {, } \\
\text { H } 4.5 \% \text {, } \\
\text { N } 11.12 \%\end{array}$ & $\begin{array}{l}\text { C } 45.83 \% \text {, } \\
\text { H } 4.17 \% \text {, } \\
\text { N } 11.51 \%\end{array}$ \\
\hline $\begin{array}{l}2 \text { after immersing } \\
\text { in benzonitrile }\end{array}$ & $\begin{array}{l}\text { Not fitting to any usual } \\
\text { formula. }\end{array}$ & ---- & ---- & $\begin{array}{l}\text { Found } \\
\text { C 46.69\%, } \\
\text { H } 4.02 \% \text {, } \\
\text { N } 11.42 \%\end{array}$ \\
\hline
\end{tabular}


IR Spectra of 1, Complexes 2-4 and the Guest Exchanged Complexes of 2:

1. IR Spectra of 1a:

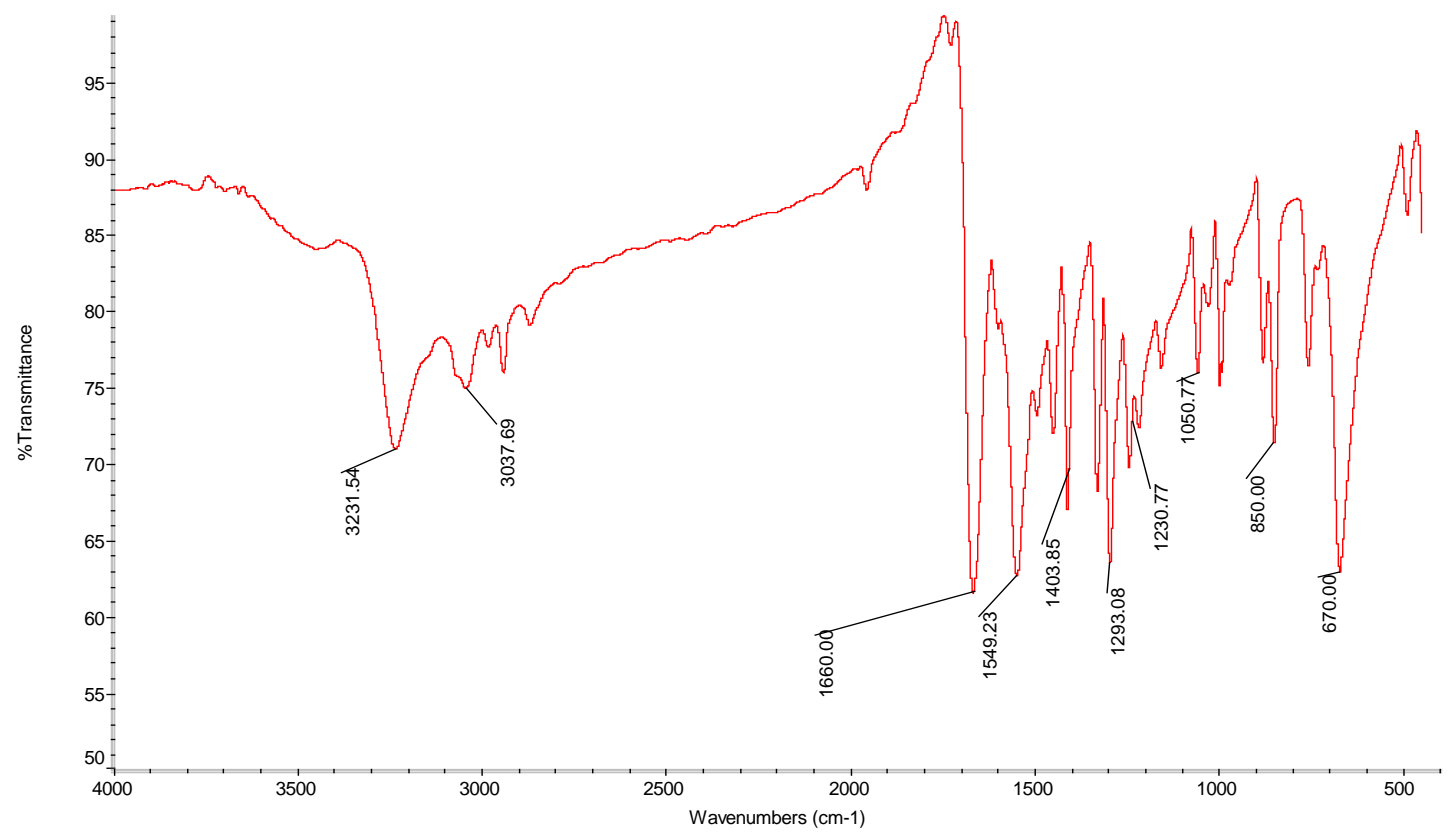

$3231 \mathrm{~cm}^{-1}$ (N-H stretch.) $3037 \mathrm{~cm}^{-1}$ (aromatic C-H stretch), $1660 \mathrm{~cm}^{-1}$ (amide C=O), $1549 \mathrm{~cm}^{-}$ ${ }^{1}$ (amide II)

2. IR Spectra of 2:

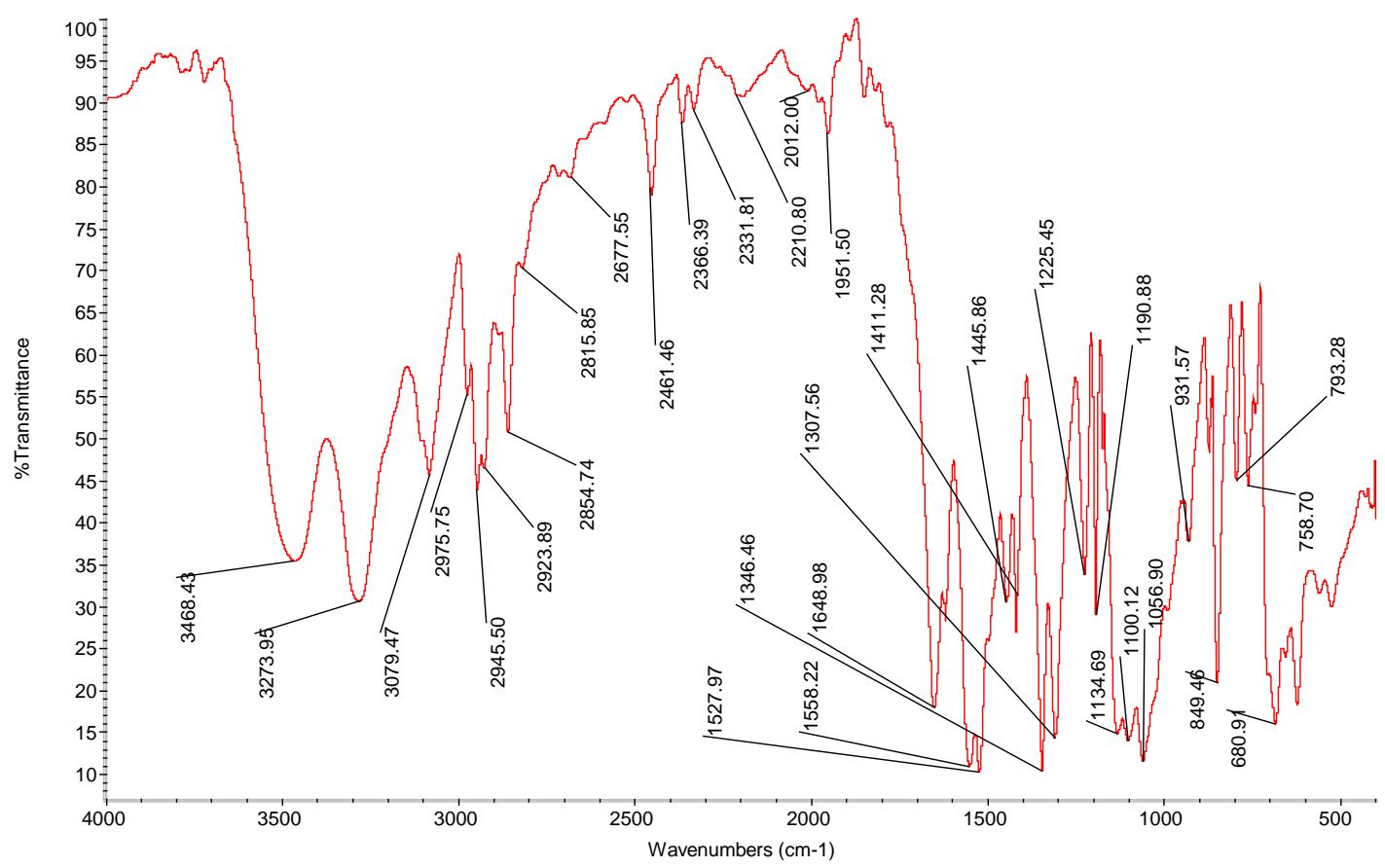

$3468 \mathrm{~cm}^{-1}$ (O-H stretch), $3273 \mathrm{~cm}^{-1}$ (N-H stretch.) $3079 \mathrm{~cm}^{-1}$ (aromatic C-H stretch), $1648 \mathrm{~cm}^{-}$ ${ }^{1}$ (amide $\left.\mathrm{C}=\mathrm{O}\right), 1558 \mathrm{~cm}^{-1}$ (amide II), $1527 \mathrm{~cm}^{-1}$ (N-O asym. stretch), $1100 \mathrm{~cm}^{-1}\left(\mathrm{ClO}_{4}\right), 1346 \mathrm{~cm}^{-}$ ${ }^{1}$ (sym. N-O stretch.), $849 \mathrm{~cm}^{-1}$ (C-N stretch) 
3. IR Spectra of 3:

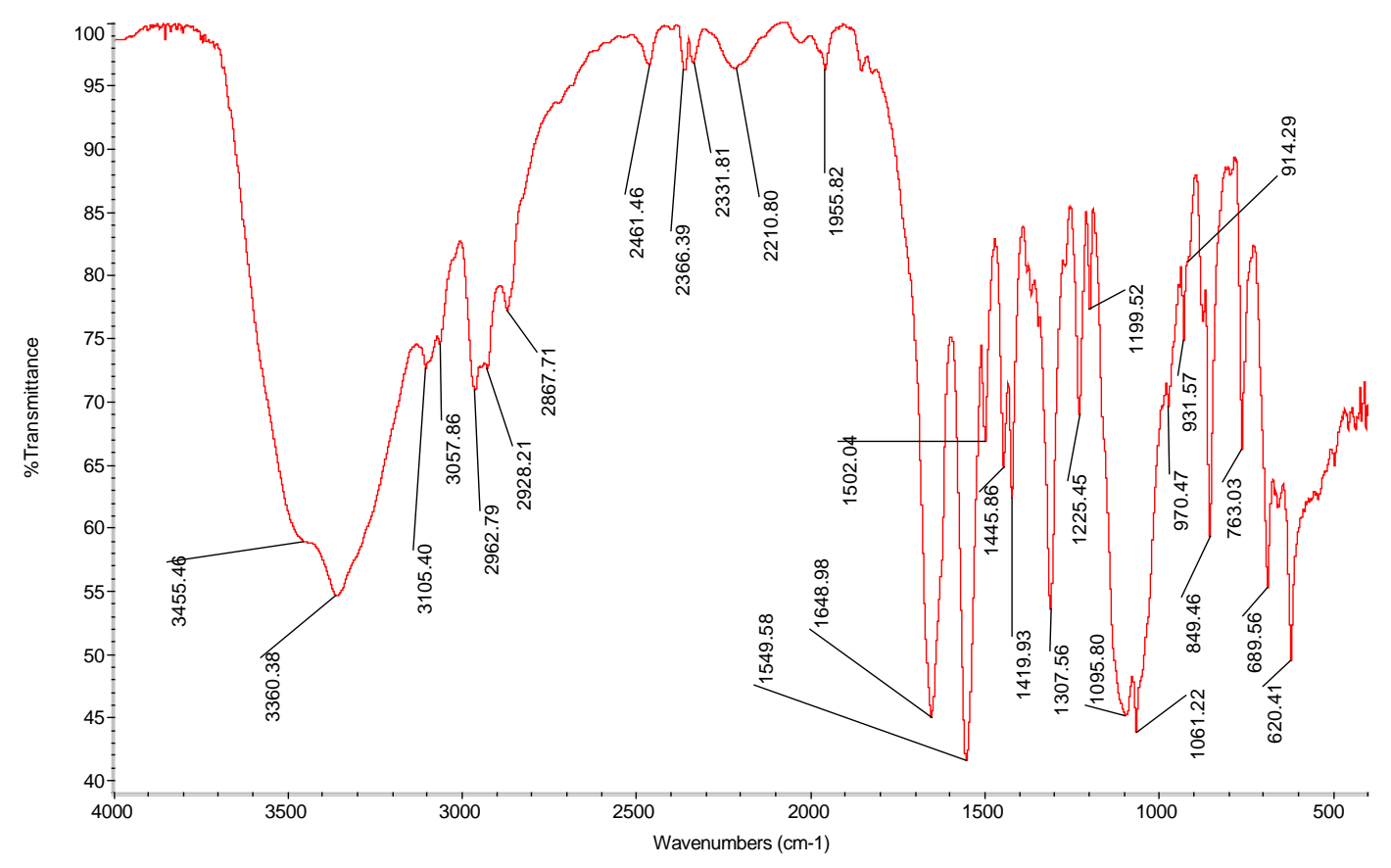

$3455 \mathrm{~cm}^{-1}$ (O-H stretch), $3360 \mathrm{~cm}^{-1}$ (N-H stretch.) $3105 \mathrm{~cm}^{-1}$ (aromatic C-H stretch), $1648 \mathrm{~cm}^{-}$ ${ }^{1}$ (amide $\mathrm{C}=\mathrm{O}$ ), $1549 \mathrm{~cm}^{-1}$ (amide II), $1095 \mathrm{~cm}^{-1}\left(\mathrm{ClO}_{4}\right)$

4. IR Spectra of 4 :

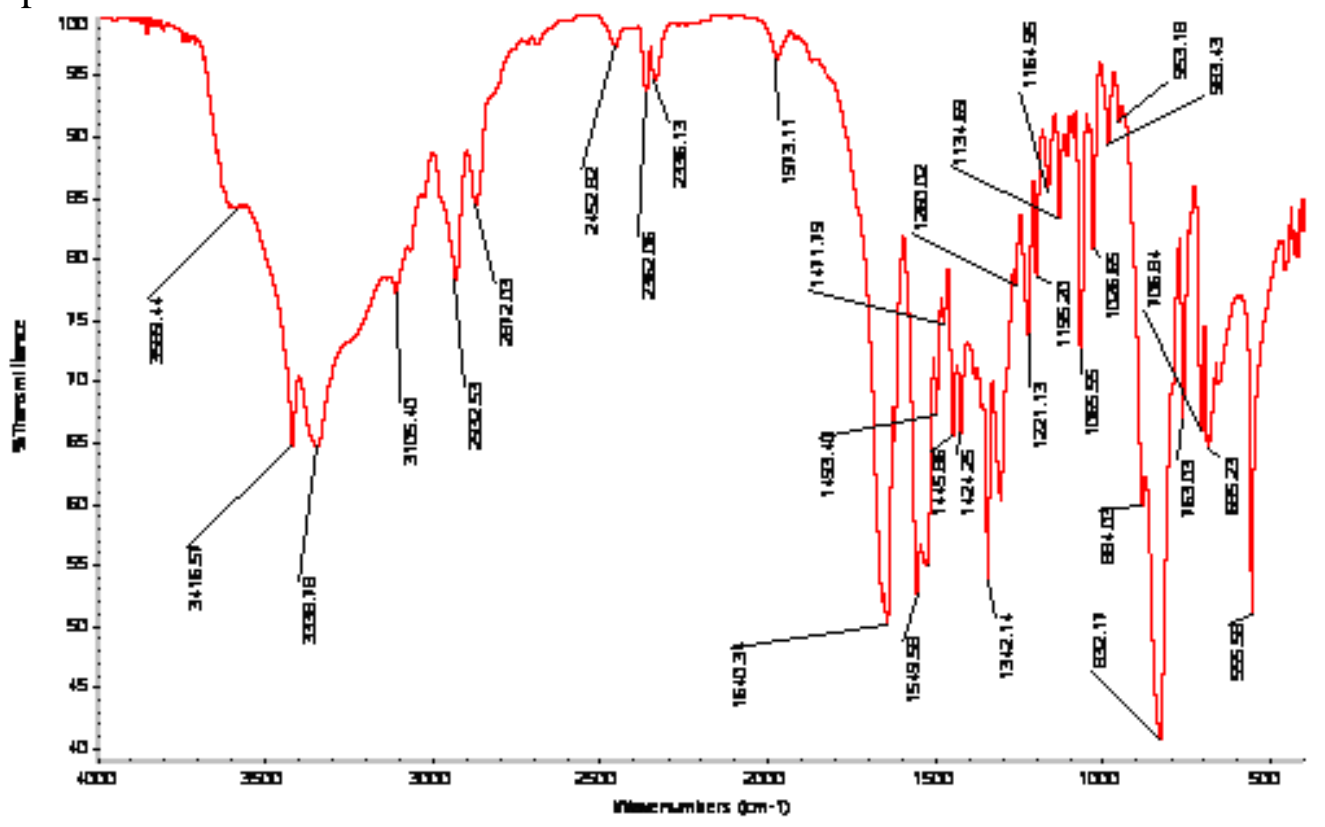

$3416 \mathrm{~cm}^{-1}$ (O-H stretch.), $3338 \mathrm{~cm}^{-1}$ (N-H stretch.) $3105 \mathrm{~cm}^{-1}$ (aromatic C-H stretch.), $1640 \mathrm{~cm}^{-}$ ${ }^{1}$ (amide $\mathrm{C}=\mathrm{O}$ ), $1549 \mathrm{~cm}^{-1}$ (amide II), $832 \mathrm{~cm}^{-1}\left(\mathrm{PF}_{6}\right), 555 \mathrm{~cm}^{-1}\left(\mathrm{PF}_{6}\right)$. 
5. IR Spectra of $\mathbf{2}$ without any Guest:

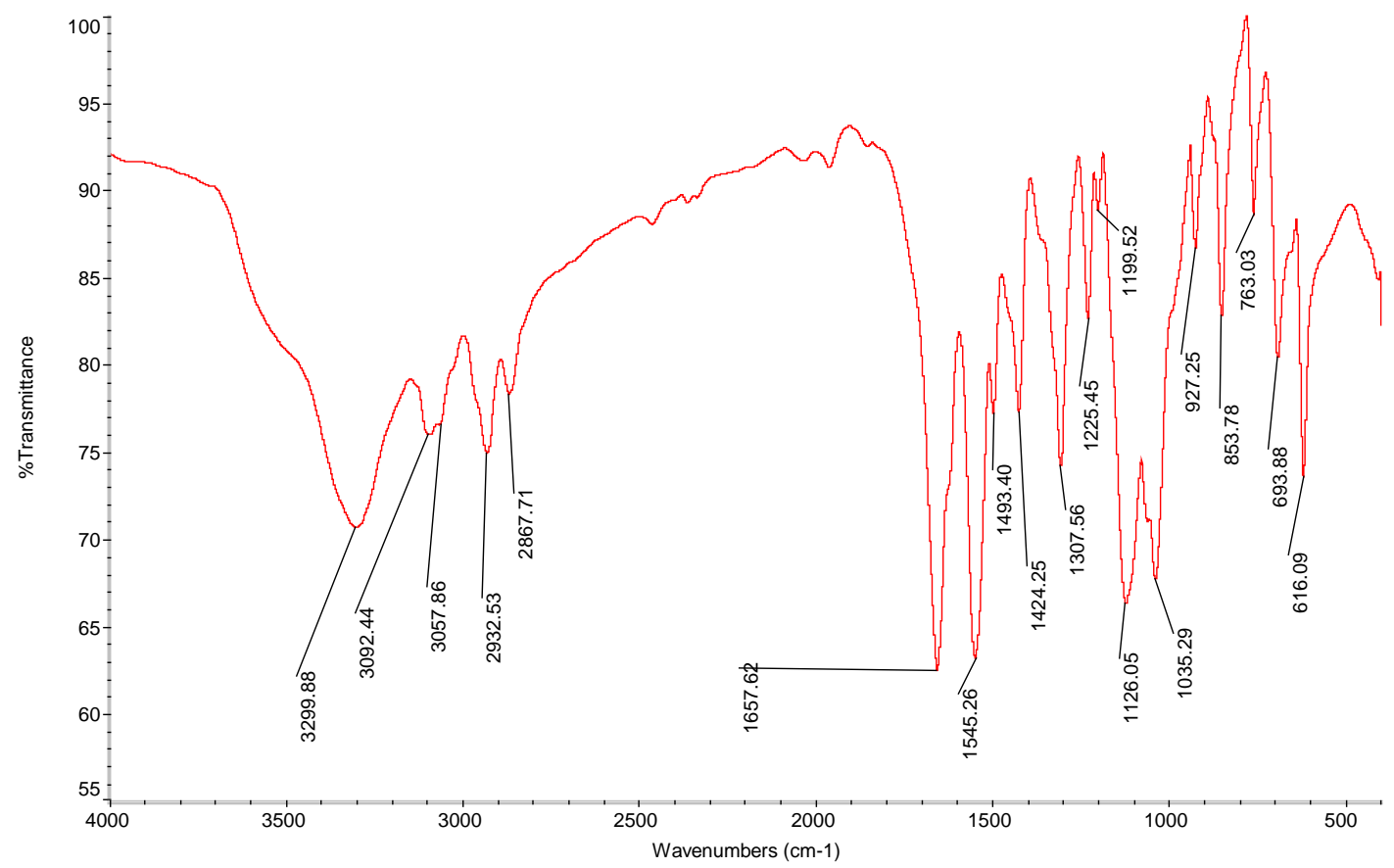

$3299 \mathrm{~cm}^{-1}$ (N-H stretch.) $3057 \mathrm{~cm}^{-1}$ (aromatic C-H stretch), $1657 \mathrm{~cm}^{-1}$ (amide C=O), $1545 \mathrm{~cm}^{-}$ ${ }^{1}$ (amide II)

6. IR Spectra of $\mathbf{2}$ after immersing the host complex back in nitrobenzene:

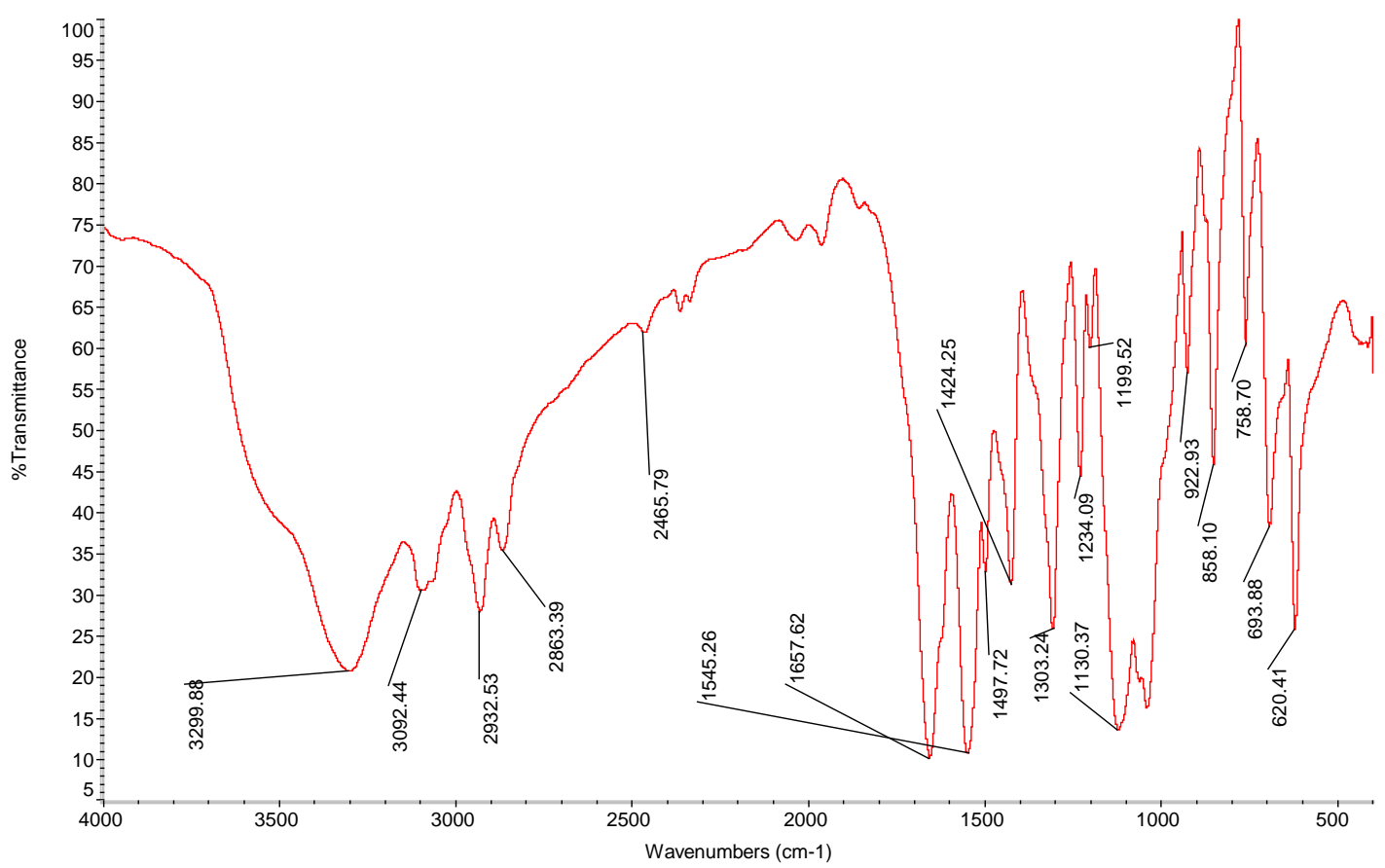

$3299 \mathrm{~cm}^{-1}$ (N-H stretch.) $3092 \mathrm{~cm}^{-1}$ (aromatic C-H stretch), $1657 \mathrm{~cm}^{-1}$ (amide C=O), $1545 \mathrm{~cm}^{-}$ (amide II) 
7. IR Spectra of guest exchanged complex of $\mathbf{2}$ with anisole:

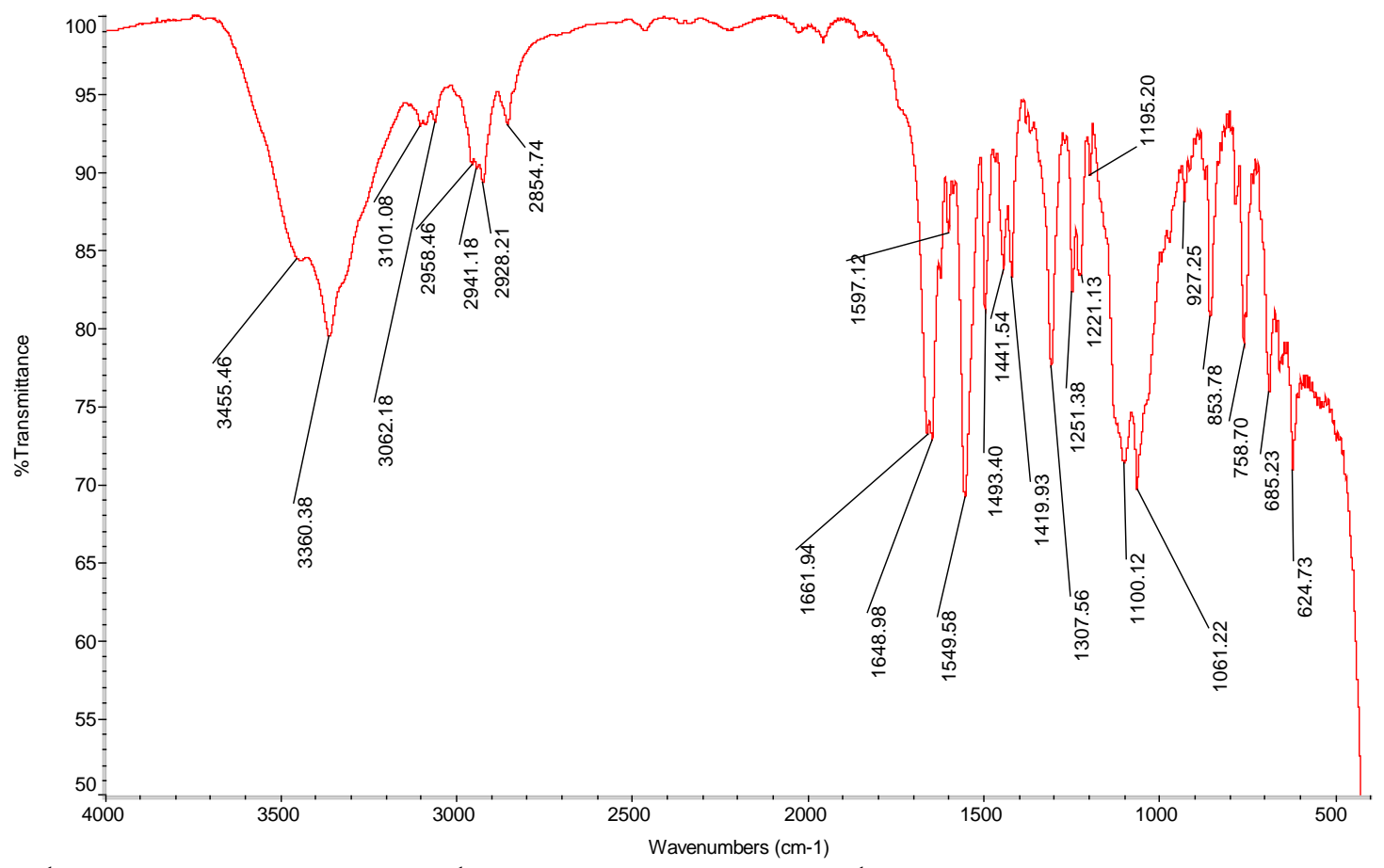

$3455 \mathrm{~cm}^{-1}$ (O-H stretch.), $3360 \mathrm{~cm}^{-1}$ (N-H stretch), $3062 \mathrm{~cm}^{-1}$ (aromatic C-H stretch), $1661 \mathrm{~cm}^{-}$ ${ }^{1}$ (amide $\mathrm{C}=\mathrm{O}$ ), $1549 \mathrm{~cm}^{-1}$ (amide II)

8. IR Spectra of Guest exchanged complex of 2 with p-xylene:

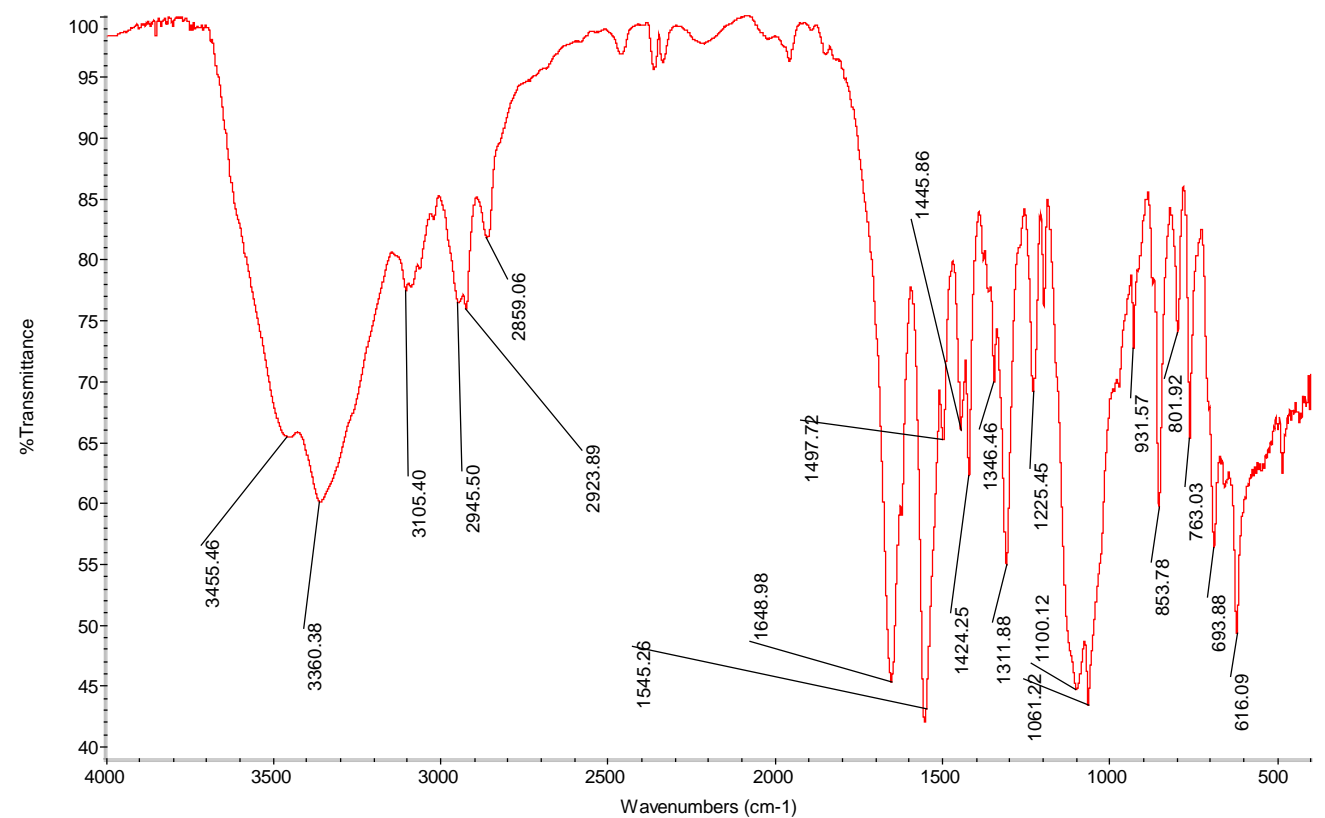

$3455 \mathrm{~cm}^{-1}$ (O-H stretch.), $3360 \mathrm{~cm}^{-1}$ (N-H stretch), $3105 \mathrm{~cm}^{-1}$ (aromatic C-H stretch), $1648 \mathrm{~cm}^{-}$ ${ }^{1}$ (amide $\mathrm{C}=\mathrm{O}$ ), $1545 \mathrm{~cm}^{-1}$ (amide II) 
9. IR Spectra of guest exchanged complex of $\mathbf{2}$ with chlorobenzene:

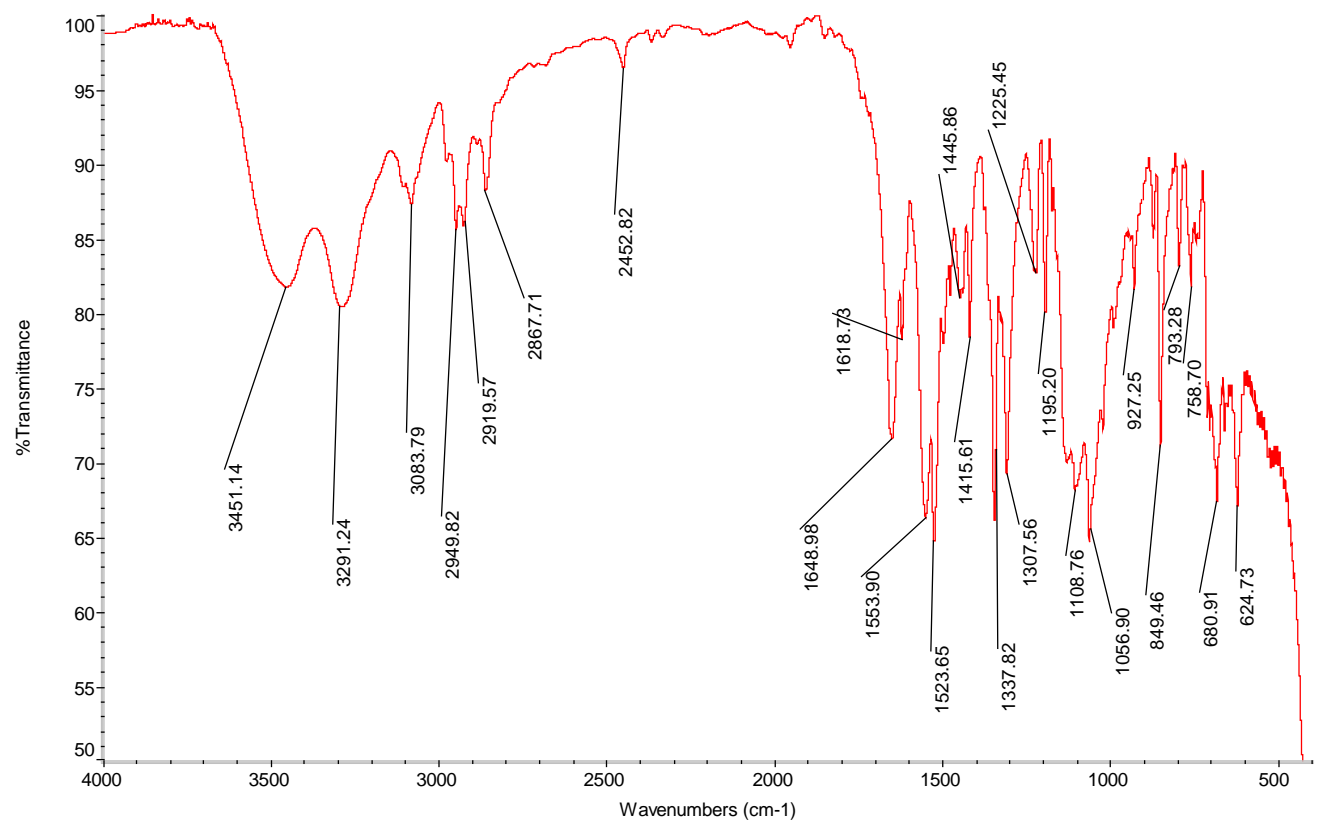

$3451 \mathrm{~cm}^{-1}$ (O-H stretch), $3291 \mathrm{~cm}^{-1}$ (N-H stretch.), $3083 \mathrm{~cm}^{-1}$ (aromatic C-H stretch), $1648 \mathrm{~cm}^{-}$ ${ }^{1}$ (amide $\mathrm{C}=\mathrm{O}$ ), $1553 \mathrm{~cm}^{-1}$ (amide II), $1523 \mathrm{~cm}^{-1}$ (N-O asym. stretch), $1337 \mathrm{~cm}^{-1}$ (sym. N-O stretch.), $849 \mathrm{~cm}^{-1}$ (C-N stretch)

10. IR Spectra of Guest Exchanged complex of 2 with benzonitrile: 


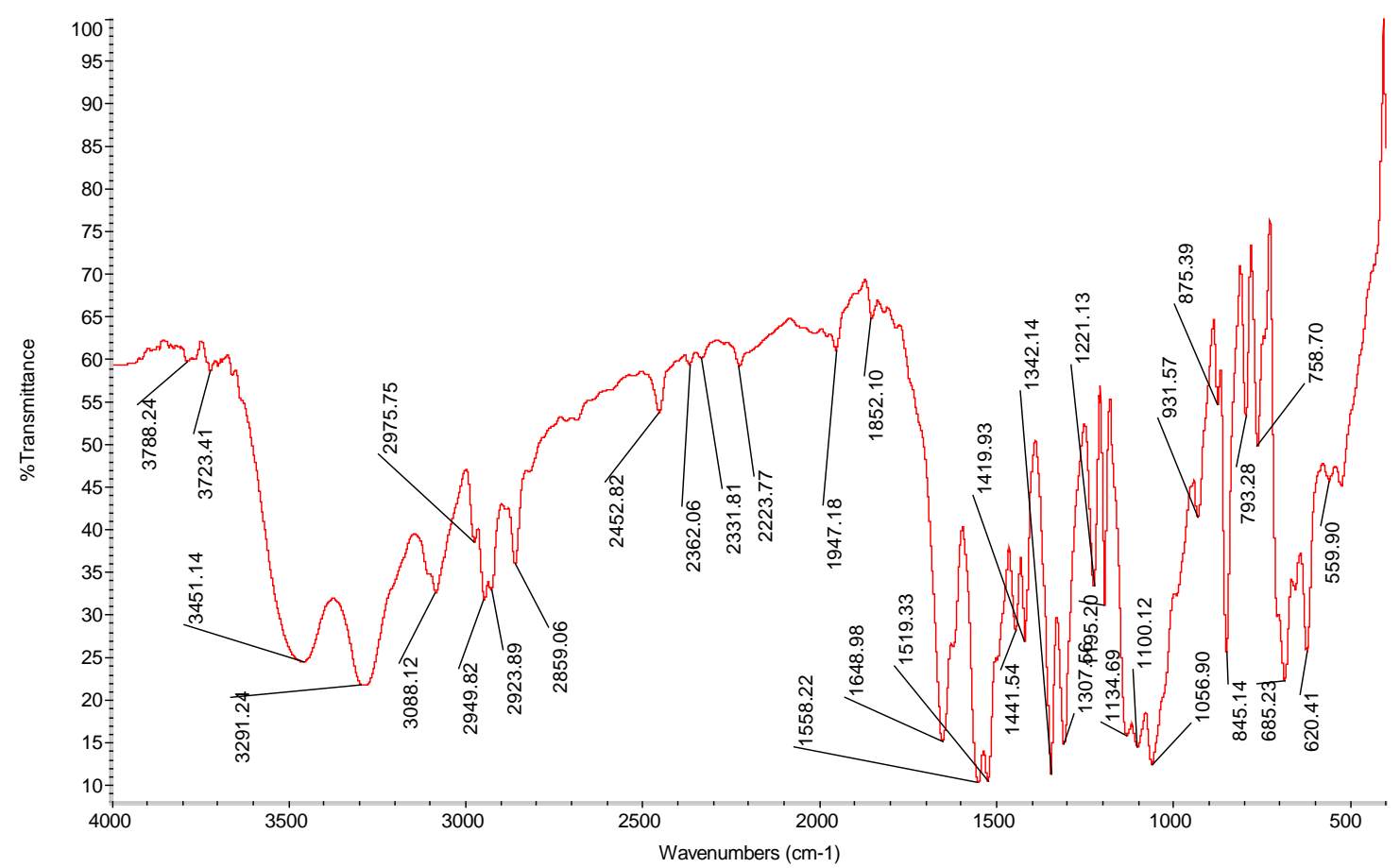

$3451 \mathrm{~cm}^{-1}$ (O-H stretch), $3291 \mathrm{~cm}^{-1}$ (N-H stretch.), $3088 \mathrm{~cm}^{-1}$ (aromatic C-H stretch), $1648 \mathrm{~cm}^{-}$ ${ }^{1}$ (amide $\left.\mathrm{C}=\mathrm{O}\right), 1558 \mathrm{~cm}^{-1}$ (amide II), $1519 \mathrm{~cm}^{-1}$ (N-O asym. stretch), $1342 \mathrm{~cm}^{-1}$ (sym. N-O stretch.), $845 \mathrm{~cm}^{-1}$ (C-N stretch)

Elemental Analysis and IR of the Guest Exchanged and removal Reactions of 2:

\begin{tabular}{|c|c|c|c|c|c|c|c|}
\hline Complex & $\begin{array}{l}\text { Elemental } \\
\text { Analysis }\end{array}$ & $\begin{array}{l}\mathrm{N}-\mathrm{H} \\
\text { Stretch }\end{array}$ & $\begin{array}{l}\text { Aroma } \\
\text { tic C- } \\
\mathrm{H}\end{array}$ & $\begin{array}{l}\text { Amide } \\
\mathrm{C}=\mathrm{O}\end{array}$ & $\begin{array}{l}\text { Amide } \\
\text { II }\end{array}$ & $\begin{array}{l}\text { Asym. } \\
\mathrm{N}-\mathrm{O}\end{array}$ & $\begin{array}{l}\text { Sym. N- } \\
\text { O }\end{array}$ \\
\hline $\begin{array}{l}\mathbf{2} \\
\mathrm{CuL}_{2}\left(\mathrm{H}_{2} \mathrm{O}\right)_{2}\left(\mathrm{ClO}_{4}\right)_{2} .3 \mathrm{NB}\end{array}$ & $\begin{array}{l}\text { Calc. } \\
\text { C } 47.48 \% \text {, } \\
\text { H } 4.35 \%, \\
\text { N } 12.18 \% \\
\text { Found } \\
\text { C } 47.34 \% \text {, } \\
\text { H } 4.11 \% \text {, } \\
\text { N } 11.83 \%\end{array}$ & $\begin{array}{l}3273 \\
\mathrm{~cm}^{-1}\end{array}$ & $\begin{array}{l}3079 \\
\mathrm{~cm}^{-1}\end{array}$ & $\begin{array}{l}1648 \\
\mathrm{~cm}^{-1}\end{array}$ & $\begin{array}{l}1558 \\
\mathrm{~cm}^{-1}\end{array}$ & $\begin{array}{l}1527 \\
\mathrm{~cm}^{-1}\end{array}$ & $\begin{array}{l}1346 \\
\mathrm{~cm}^{-1}\end{array}$ \\
\hline $\begin{array}{l}2 \text { (without Guest) } \\
\mathrm{CuL}_{2}\left(\mathrm{H}_{2} \mathrm{O}\right)_{2}\left(\mathrm{ClO}_{4}\right)_{2}\end{array}$ & $\begin{array}{l}\text { Calc. } \\
\text { C } 42.94 \% \\
\text { H } 4.50 \% \\
\text { N } 12.52 \% \\
\text { Found } \\
\text { C } 42.07 \% \\
\text { H } 4.03 \% \\
\text { N } 12.0 \% \\
\end{array}$ & 3299 & 3092 & 1657 & 1545 & absent & absent \\
\hline
\end{tabular}




\begin{tabular}{|c|c|c|c|c|c|c|c|}
\hline $\begin{array}{l}\text { After immersing } 2 \\
\text { (without Guest) in } \\
\text { Nitrobenzene for one } \\
\text { week } \\
\mathrm{CuL}_{2}\left(\mathrm{H}_{2} \mathrm{O}\right)_{2}\left(\mathrm{ClO}_{4}\right)_{2}\end{array}$ & $\begin{array}{l}\text { Calc. } \\
\text { C } 42.94 \% \\
\text { H } 4.50 \% \\
\text { N } 12.52 \% \\
\text { Found } \\
\text { C } 42.15 \% \\
\text { H } 4.32 \% \\
\text { N } 12.25 \%\end{array}$ & 3299 & 3092 & 1657 & 1545 & absent & absent \\
\hline $\begin{array}{l}2 \text { after immersing in } \\
\text { Anisole } \\
\mathrm{CuL}_{2}\left(\mathrm{H}_{2} \mathrm{O}\right)_{2}(\text { Anisole })_{2}(\mathrm{Cl} \\
\left.\mathrm{O}_{4}\right)_{2}\end{array}$ & $\begin{array}{l}\text { Calc. } \\
\text { C } 49.7 \%, \\
\text { H } 5.04 \%, \\
\text { N } 10.08 \% \\
\text { Found } \\
\text { C } 49.59 \% \text {, } \\
\text { H } 4.97 \%, \\
\text { N } 9.9 \%\end{array}$ & 3360 & 3062 & 1661 & 1549 & absent & absent \\
\hline $\begin{array}{l}2 \text { after immersing in } \mathrm{p}- \\
\text { xylene } \\
\mathrm{CuL}_{2}\left(\mathrm{H}_{2} \mathrm{O}\right)_{2}(\text { xylene })_{1.5}(\mathrm{Cl} \\
\left.\mathrm{O}_{4}\right)_{2}\end{array}$ & $\begin{array}{l}\text { Calc. } \\
\text { C 50.1\%, } \\
\text { H } 5.22 \% \text {, } \\
\text { N } 10.63 \% \\
\text { Found } \\
\text { C } 49.58 \% \text {, } \\
\text { H } 5.01 \% \text {, } \\
\text { N } 10.48 \%\end{array}$ & 3360 & 3105 & 1648 & 1545 & absent & absent \\
\hline $\begin{array}{l}2 \text { after immersing in } \\
\text { Chlorobenzene } \\
\mathrm{CuL}_{2}\left(\mathrm{H}_{2} \mathrm{O}\right)_{2}(\text { Chlorobenze } \\
\text { ne })_{1}\left(\mathrm{ClO}_{4}\right)_{2}\end{array}$ & $\begin{array}{l}\text { Calc. } \\
\text { C } 45.29 \% \text {, } \\
\text { H } 4.5 \%, \\
\text { N } 11.12 \% \\
\text { Found } \\
\text { C } 45.83 \% \text {, } \\
\text { H } 4.17 \% \text {, } \\
\text { N } 11.51 \%\end{array}$ & 3299 & 3083 & 1661 & 1549 & $\begin{array}{l}1523 \\
\text { (prese } \\
\text { nt) }\end{array}$ & $\begin{array}{l}1337 \\
\text { (present) }\end{array}$ \\
\hline $\begin{array}{l}\mathbf{2} \text { after immersing in } \\
\text { Benzonitrile }\end{array}$ & $\begin{array}{l}\text { Found } \\
\text { C 46.69\%, } \\
\text { H } 4.02 \% \text {, } \\
\text { N } 11.42 \%\end{array}$ & 3291 & 3088 & 1648 & 1558 & $\begin{array}{l}1519 \\
\text { (prese } \\
\text { nt) }\end{array}$ & $\begin{array}{l}1342 \\
\text { (present) }\end{array}$ \\
\hline
\end{tabular}

\section{TG Analysis of 2 and 3.}


TGA of 2:

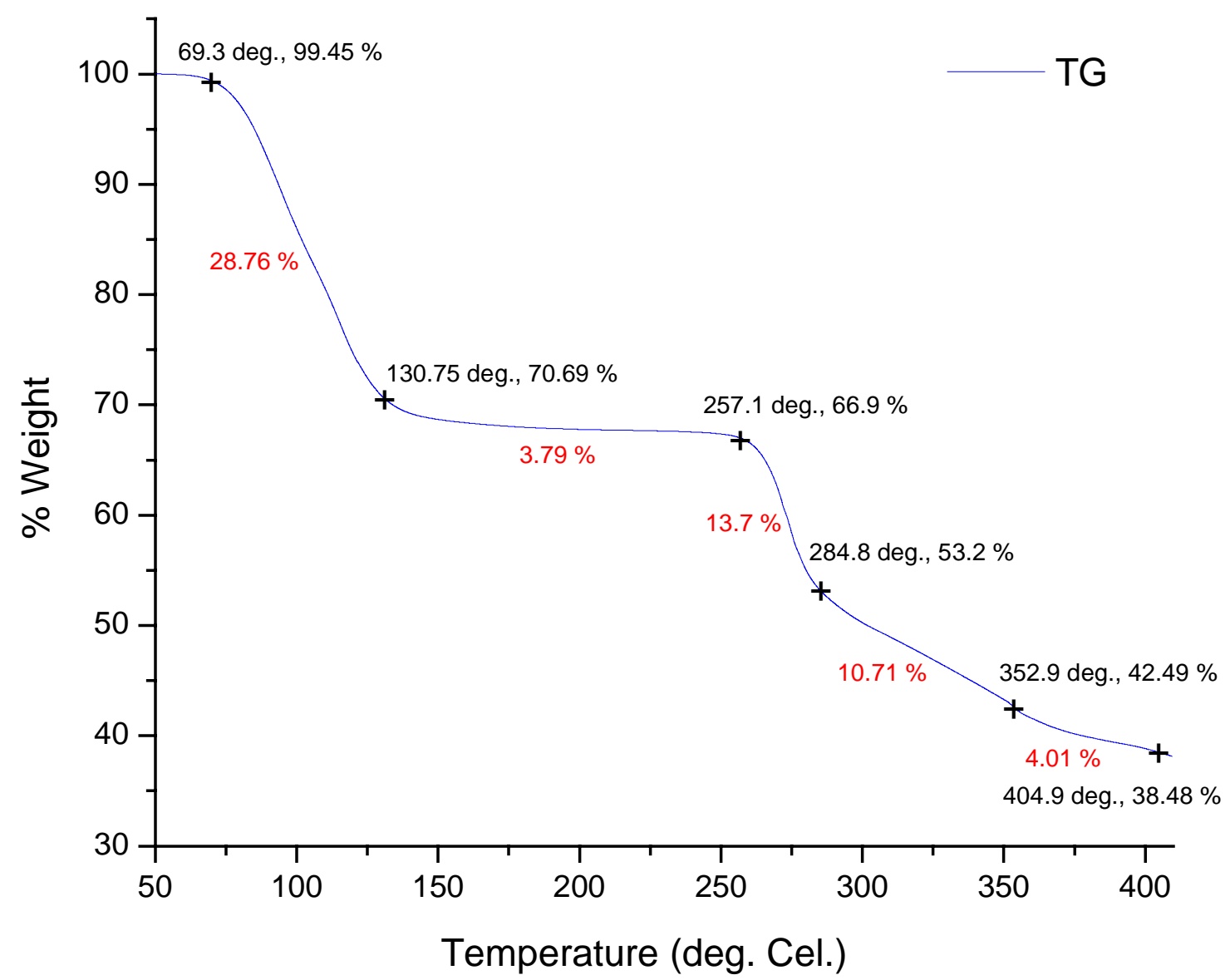

Temperature Range: 50 to $400{ }^{\circ} \mathrm{C}$; Rate of Heating: $5{ }^{\circ} \mathrm{C} / \mathrm{min}$.

Weight loss of $29.31 \%$ observed at $130{ }^{\circ} \mathrm{C}$ corresponds to the loss of three nitrobenzene molecules (Calculated: $29.2 \%$ ). From $130{ }^{\circ} \mathrm{C}$ to $257^{\circ} \mathrm{C}$, weight loss of $3.79 \%$ is due to the loss of two coordinated water molecules (Calculated: $4.02 \%$ ). The $66.9 \%$ weight of the complex at $257^{\circ} \mathrm{C}$ is due to the presence of the host framework $\left(\mathrm{Cu} \mathrm{L}_{2}\left(\mathrm{ClO}_{4}\right)_{2}\right)$. (Calculated: $\left.67.94 \%\right)$. The network is stable up to $257^{\circ} \mathrm{C}$. 
TGA of 3:

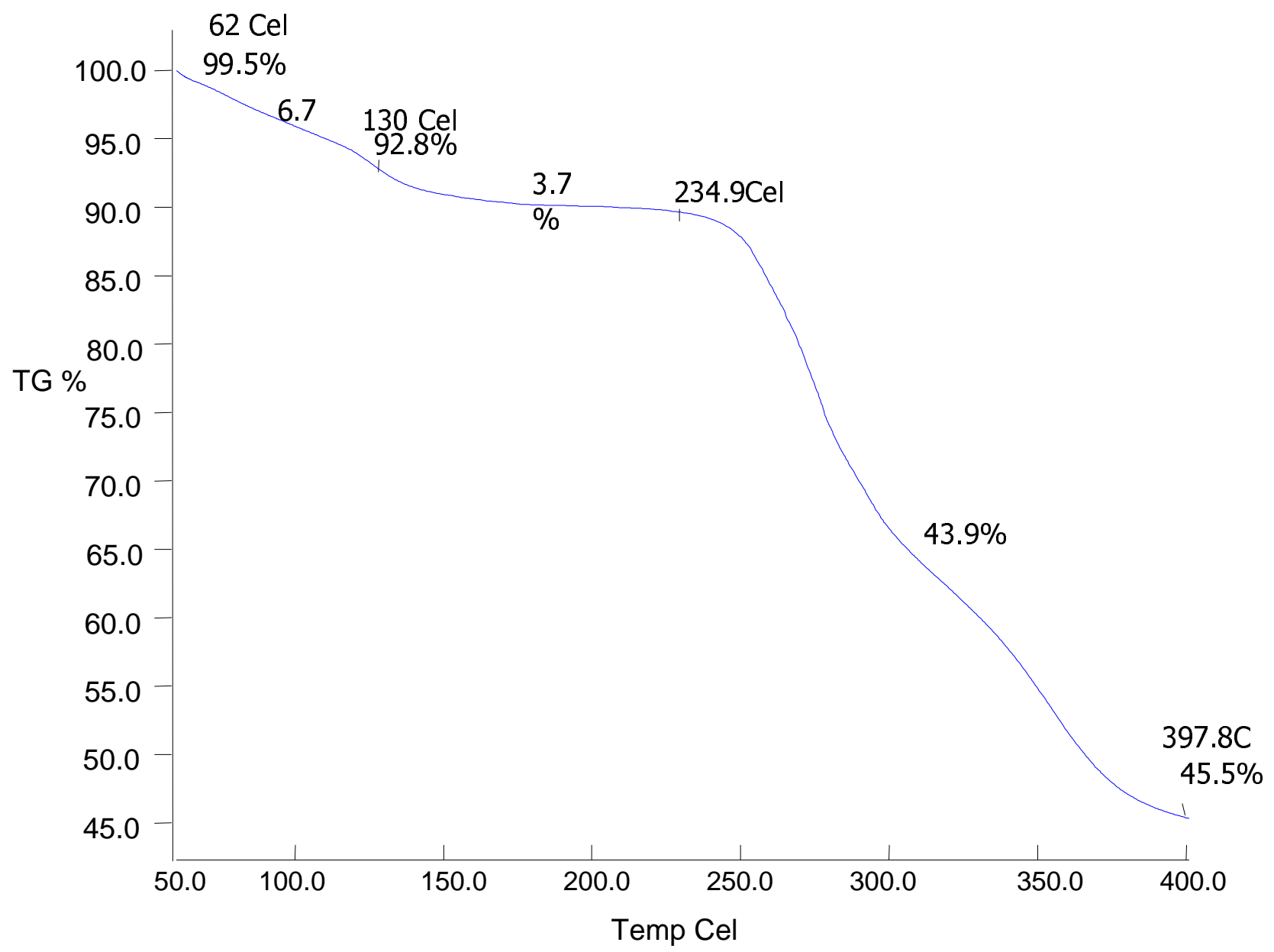

Temperature Range: 50 to $400{ }^{\circ} \mathrm{C}$; Rate of Heating: $5{ }^{\circ} \mathrm{C} / \mathrm{min}$.

The TGA of 3 shows that there is a weight loss of $7.2 \%$ from $62.9^{\circ} \mathrm{C}$ to $130^{\circ} \mathrm{C}$ which corresponds to the loss of one ethanol and one water molecule (Calculated: 6.68\%). The weight loss of 3.75\% is observed in the temperature range of $130^{\circ} \mathrm{C}$ to $235^{\circ} \mathrm{C}$ which corresponds to the loss of two coordinated water molecules (Calculated: $4.02 \%$ ). 


\section{Powder XRD Pattern of the Guest Exchanged Complexes of 2:}

1. Powder XRD Spectra of $\mathbf{2}$ and guest exchanged complex of $\mathbf{2}$ with anisole

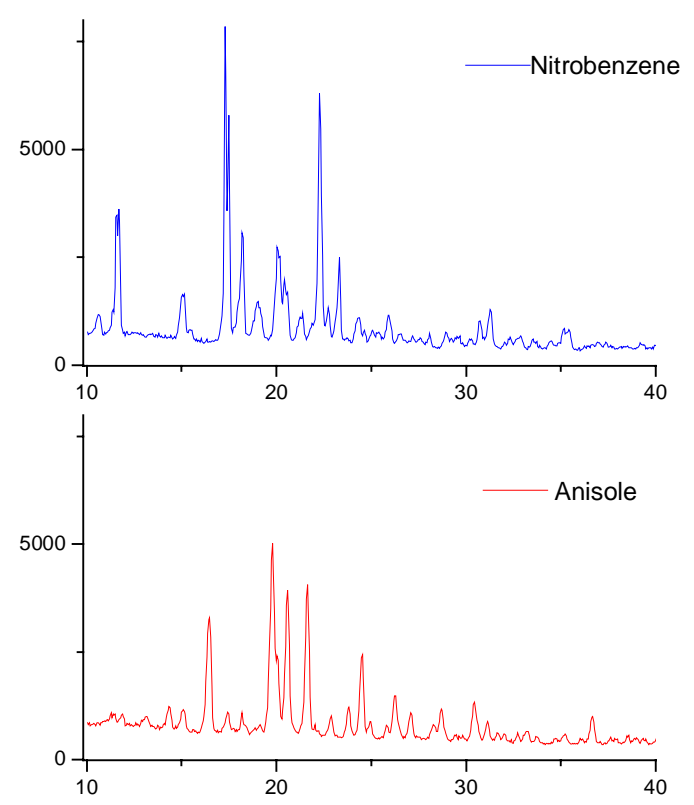

2. Powder XRD Spectra of $\mathbf{2}$ and guest exchanged complex of $\mathbf{2}$ with p-xylene: 


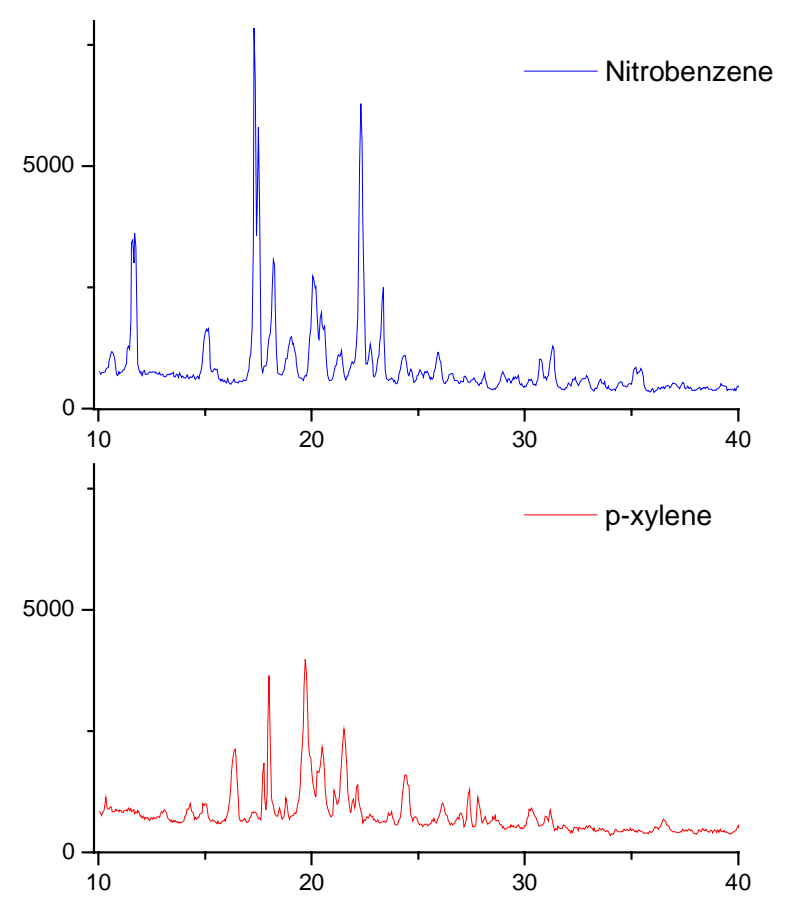

3. Powder XRD Spectra of $\mathbf{2}$ and guest exchanged complex of $\mathbf{2}$ with Chlorobenzene:

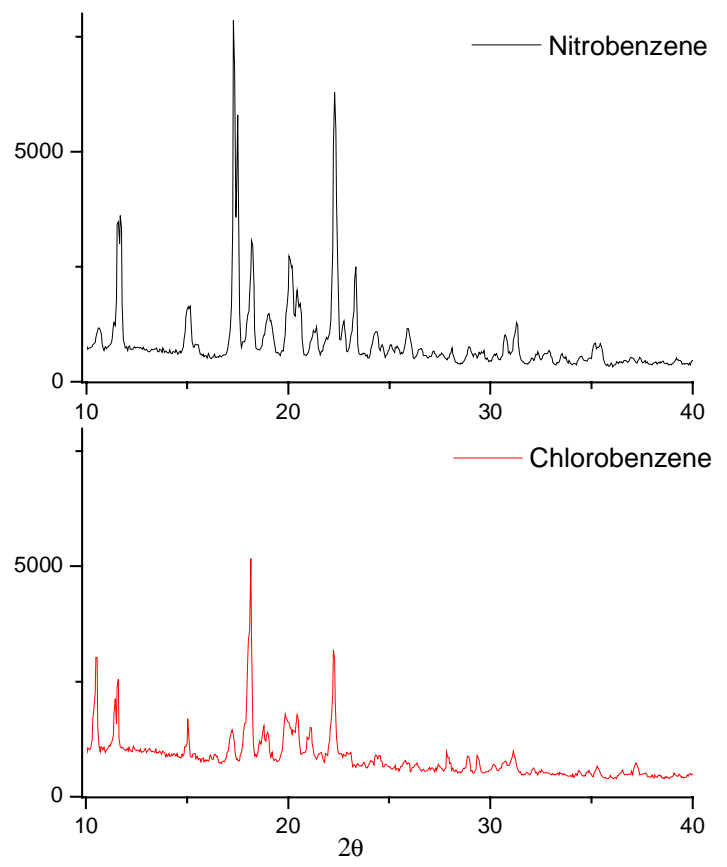

4. Powder XRD Spectra of $\mathbf{2}$ and guest exchanged complex of $\mathbf{2}$ with Benzonitrile: 

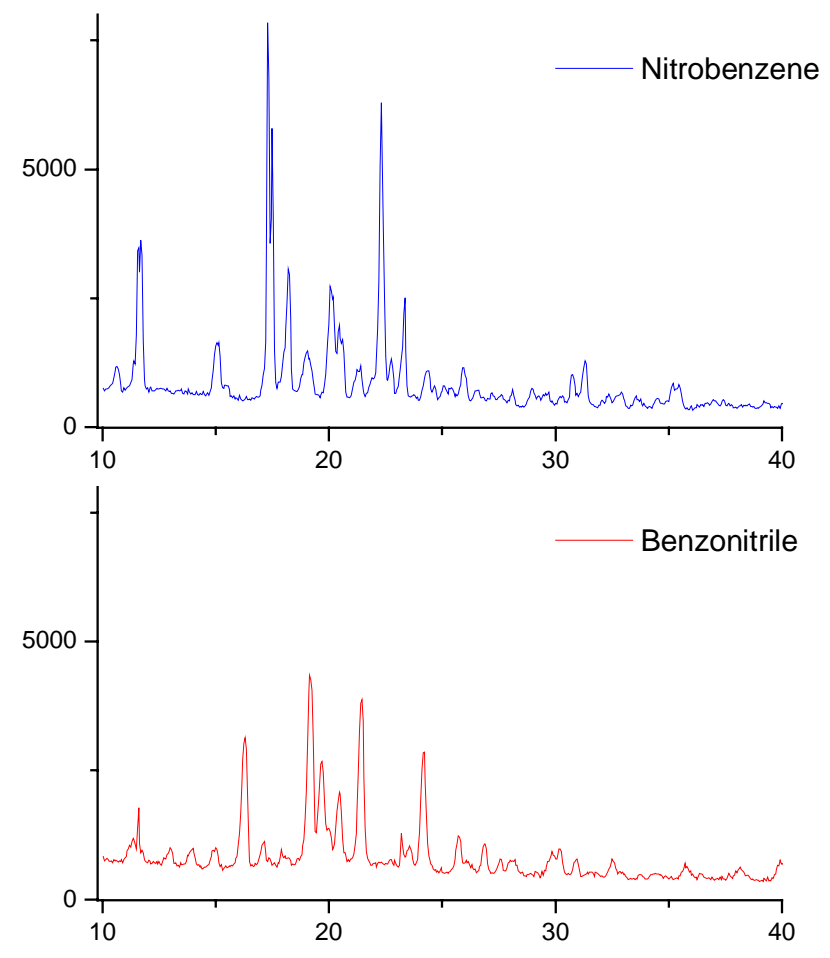

5. Powder XRD Spectra of $\mathbf{2}$ and $\mathbf{2}$ (without any guest):

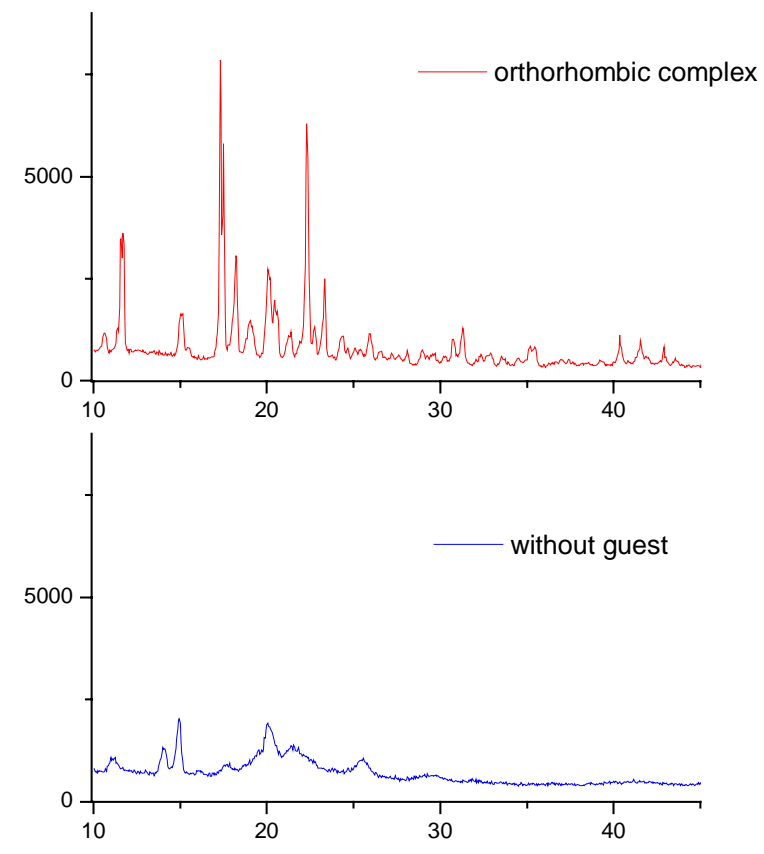

6. Powder XRD Spectra of $\mathbf{2}$ (without any guest) and the complex obtained by immersing back the crystals of $\mathbf{2}$ (without guest) in nitrobenzene: 


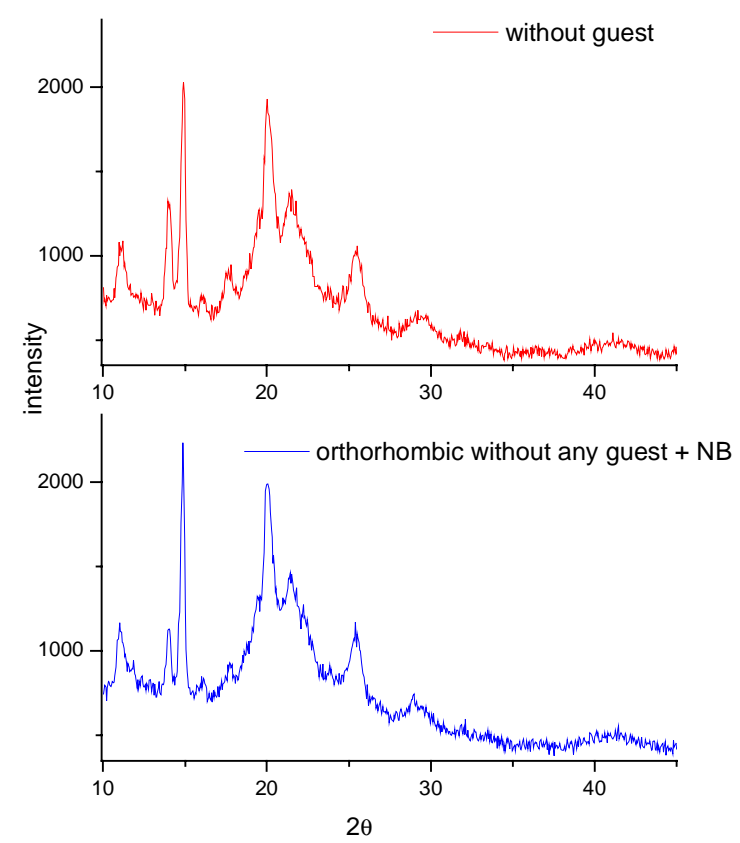


Table 1. Crystal data and structure refinement for $\mathbf{2}$.

Identification code

Empirical formula

Formula weight

Temperature

Wavelength

Crystal system

Space group

Unit cell dimensions

Volume

Z

Density (calculated)

Absorption coefficient

$\mathrm{F}(000)$

Crystal size

Theta range for data collection

Index ranges

Reflections collected

Independent reflections

Completeness to theta $=24.96^{\circ}$

Absorption correction

Refinement method

Data / restraints / parameters

Goodness-of-fit on $\mathrm{F}^{2}$

Final $\mathrm{R}$ indices [I $>2 \operatorname{sigma}(\mathrm{I})]$

$\mathrm{R}$ indices (all data)

Largest diff. peak and hole
2

C50 H55 Cl2 Cu N11 O20

1264.49

293(2) K

$0.71073 \AA$

Orthorhombic

$\mathrm{Pbcn}$

$\mathrm{a}=18.567(4) \AA \quad \alpha=90^{\circ}$.

$\mathrm{b}=9.934(2) \AA \quad \beta=90^{\circ}$.

$\mathrm{c}=30.764(6) \AA \quad \gamma=90^{\circ}$.

5674(2) $\AA^{3}$

4

$1.480 \mathrm{Mg} / \mathrm{m}^{3}$

$0.565 \mathrm{~mm}^{-1}$

2620

$0.20 \times 0.15 \times 0.10 \mathrm{~mm}^{3}$

1.72 to $24.96^{\circ}$.

$-22<=\mathrm{h}<=0,-11<=\mathrm{k}<=0,-36<=\mathrm{l}<=0$

4535

$4535[\mathrm{R}(\mathrm{int})=0.0000]$

$91.0 \%$

None

Full-matrix least-squares on $\mathrm{F}^{2}$

4535 / 6 / 455

1.042

$\mathrm{R} 1=0.0839, \mathrm{wR} 2=0.1669$

$\mathrm{R} 1=0.1452, \mathrm{wR} 2=0.2002$

0.469 and -0.377 e. $\AA^{-3}$ 
Table 2. Atomic coordinates ( $\times 10^{4}$ ) and equivalent isotropic displacement parameters $\left(\AA^{2} \times 10^{3}\right)$ for 2. $U(e q)$ is defined as one third of the trace of the orthogonalized $U^{\mathrm{ij}}$ tensor.

\begin{tabular}{|c|c|c|c|c|}
\hline & $\mathrm{x}$ & $\mathrm{y}$ & $\mathrm{z}$ & $\mathrm{U}(\mathrm{eq})$ \\
\hline $\mathrm{Cu}(1)$ & 0 & $6788(1)$ & 2500 & $36(1)$ \\
\hline $\mathrm{O}(1 \mathrm{~W})$ & 0 & $9229(7)$ & 2500 & $71(2)$ \\
\hline $\mathrm{O}(2 \mathrm{~W})$ & 0 & 4187(7) & 2500 & $103(3)$ \\
\hline $\mathrm{N}(21)$ & $-790(3)$ & $6749(5)$ & 2041(2) & $37(1)$ \\
\hline $\mathrm{O}(26)$ & $-2722(2)$ & $5423(5)$ & $992(2)$ & $56(1)$ \\
\hline $\mathrm{N}(31)$ & $-2370(3)$ & $8536(12)$ & $-836(2)$ & $83(3)$ \\
\hline $\mathrm{N}(32)$ & $-2644(3)$ & $7653(6)$ & $864(2)$ & $46(1)$ \\
\hline $\mathrm{O}(16)$ & $-2263(5)$ & $6649(9)$ & $-1011(3)$ & $47(3)$ \\
\hline $\mathrm{C}(11)$ & $-3107(7)$ & $7966(15)$ & $-1424(4)$ & $35(2)$ \\
\hline $\mathrm{C}(12)$ & $-3118(7)$ & $7076(16)$ & $-1777(4)$ & $39(3)$ \\
\hline$C(15)$ & $-3630(11)$ & $8890(20)$ & $-1385(4)$ & $48(5)$ \\
\hline$C(16)$ & $-2557(6)$ & $7732(14)$ & $-1068(5)$ & $36(3)$ \\
\hline $\mathrm{O}(16 \mathrm{~A})$ & $-2582(5)$ & $10385(9)$ & $-921(3)$ & $54(3)$ \\
\hline $\mathrm{C}(11 \mathrm{~A})$ & $-3193(9)$ & $8945(17)$ & $-1375(5)$ & $45(4)$ \\
\hline $\mathrm{C}(12 \mathrm{~A})$ & $-3130(7)$ & $7824(15)$ & $-1666(5)$ & $36(2)$ \\
\hline$C(15 A)$ & $-3798(8)$ & 9714(19) & $-1424(4)$ & $52(4)$ \\
\hline$C(16 A)$ & $-2676(7)$ & $9230(10)$ & $-1024(4)$ & $36(3)$ \\
\hline $\mathrm{N}(11)$ & $-4214(3)$ & 8291(7) & $-2032(2)$ & $56(2)$ \\
\hline$C(13)$ & $-3672(4)$ & $7408(10)$ & $-2034(3)$ & $73(3)$ \\
\hline $\mathrm{C}(14)$ & $-4240(4)$ & $9224(12)$ & $-1724(2)$ & $91(4)$ \\
\hline $\mathrm{C}(21)$ & $-1880(3)$ & $6662(6)$ & $1419(2)$ & $35(1)$ \\
\hline $\mathrm{C}(22)$ & $-1394(3)$ & $7692(7)$ & $1435(2)$ & $41(2)$ \\
\hline$C(23)$ & $-855(3)$ & $7694(6)$ & $1739(2)$ & $40(1)$ \\
\hline $\mathrm{C}(24)$ & $-1288(3)$ & $5738(6)$ & 2032(2) & $44(2)$ \\
\hline$C(25)$ & $-1818(3)$ & $5661(6)$ & $1730(2)$ & $38(1)$ \\
\hline$C(26)$ & $-2458(3)$ & $6539(7)$ & $1075(2)$ & $37(1)$ \\
\hline $\mathrm{C}(31)$ & $-1874(4)$ & $8585(8)$ & $-470(2)$ & $58(2)$ \\
\hline $\mathrm{C}(32)$ & $-2182(4)$ & 7834(9) & $-71(2)$ & $64(2)$ \\
\hline $\mathrm{C}(33)$ & $-2845(4)$ & $8500(9)$ & $123(2)$ & $64(2)$ \\
\hline $\mathrm{C}(34)$ & $-3140(4)$ & 7604(9) & $495(2)$ & $63(2)$ \\
\hline $\mathrm{N}(100)$ & $149(4)$ & $8753(8)$ & $412(3)$ & $75(2)$ \\
\hline
\end{tabular}




\begin{tabular}{lcccc}
$\mathrm{O}(101)$ & $713(4)$ & $8155(9)$ & $326(3)$ & $122(3)$ \\
$\mathrm{O}(102)$ & $-367(4)$ & $8740(8)$ & $176(2)$ & $102(2)$ \\
$\mathrm{C}(101)$ & $156(4)$ & $9610(8)$ & $798(2)$ & $54(2)$ \\
$\mathrm{C}(102)$ & $-465(4)$ & $10361(9)$ & $883(3)$ & $69(2)$ \\
$\mathrm{C}(103)$ & $-470(5)$ & $11181(11)$ & $1244(3)$ & $86(3)$ \\
$\mathrm{C}(104)$ & $132(5)$ & $11238(9)$ & $1509(3)$ & $77(3)$ \\
$\mathrm{C}(105)$ & $723(5)$ & $10468(9)$ & $1420(3)$ & $70(2)$ \\
$\mathrm{C}(106)$ & $739(4)$ & $9673(8)$ & $1074(2)$ & $58(2)$ \\
$\mathrm{N}(1 \mathrm{~A})$ & $392(13)$ & $5250(20)$ & $4528(6)$ & $84(6)$ \\
$\mathrm{C}(1 \mathrm{~A})$ & $514(11)$ & $5439(19)$ & $4924(13)$ & $110(12)$ \\
$\mathrm{C}(2 \mathrm{~A})$ & $811(9)$ & $5737(18)$ & $4435(4)$ & $122(6)$ \\
$\mathrm{C}(3 \mathrm{~A})$ & $276(10)$ & $4760(20)$ & $4214(8)$ & $76(7)$ \\
$\mathrm{C}(4 \mathrm{~A})$ & $-169(9)$ & $4191(15)$ & $4287(4)$ & $123(5)$ \\
$\mathrm{C}(5 \mathrm{~A})$ & $-525(9)$ & $4023(17)$ & $4705(6)$ & $71(5)$ \\
$\mathrm{C}(6 \mathrm{~A})$ & 0 & 5000 & 5000 & $111(6)$ \\
$\mathrm{Cl}(1)$ & $1581(1)$ & $1799(2)$ & $2574(1)$ & $74(1)$ \\
$\mathrm{O}(301)$ & $1321(8)$ & $2762(12)$ & $2261(4)$ & $154(5)$ \\
$\mathrm{O}(302)$ & $2045(6)$ & $899(12)$ & $2344(4)$ & $149(4)$ \\
$\mathrm{O}(304)$ & $870(7)$ & $1680(30)$ & $2564(6)$ & $201(8)$ \\
$\mathrm{O}(305)$ & $1828(7)$ & $2446(9)$ & $2932(4)$ & $197(6)$ \\
$\mathrm{O}(306)$ & $1366(13)$ & $468(17)$ & $2718(7)$ & $184(8)$ \\
\hline
\end{tabular}

Table 3. Bond lengths [ $[\AA]$ and angles $\left[{ }^{\circ}\right]$ for 2.

\begin{tabular}{llll}
\hline $\mathrm{Cu}(1)-\mathrm{N}(21) \# 1$ & $2.035(5)$ & $\mathrm{N}(32)-\mathrm{C}(26)$ & $1.328(8)$ \\
$\mathrm{Cu}(1)-\mathrm{N}(21)$ & $2.035(5)$ & $\mathrm{N}(32)-\mathrm{C}(34)$ & $1.464(8)$ \\
$\mathrm{Cu}(1)-\mathrm{N}(11) \# 2$ & $2.052(5)$ & $\mathrm{O}(16)-\mathrm{C}(16)$ & $1.220(15)$ \\
$\mathrm{Cu}(1)-\mathrm{N}(11) \# 3$ & $\mathrm{C}(11)-\mathrm{C}(15)$ & $1.34(2)$ \\
$\mathrm{Cu}(1)-\mathrm{O}(1 \mathrm{~W})$ & $2.052(5)$ & $\mathrm{C}(11)-\mathrm{C}(12)$ & $1.40(2)$ \\
$\mathrm{N}(21)-\mathrm{C}(23)$ & $2.425(7)$ & $\mathrm{C}(11)-\mathrm{C}(16)$ & $1.514(16)$ \\
$\mathrm{N}(21)-\mathrm{C}(24)$ & $1.327(7)$ & $\mathrm{C}(12)-\mathrm{C}(13)$ & $1.339(16)$ \\
$\mathrm{O}(26)-\mathrm{C}(26)$ & $1.366(8)$ & $\mathrm{C}(15)-\mathrm{C}(14)$ & $1.576(16)$ \\
$\mathrm{N}(31)-\mathrm{C}(16 \mathrm{~A})$ & $1.239(8)$ & $\mathrm{O}(16 \mathrm{~A})-\mathrm{C}(16 \mathrm{~A})$ & $1.203(13)$ \\
$\mathrm{N}(31)-\mathrm{C}(16)$ & $1.065(13)$ & $\mathrm{C}(11 \mathrm{~A})-\mathrm{C}(15 \mathrm{~A})$ & $1.37(2)$ \\
$\mathrm{N}(31)-\mathrm{C}(31)$ & $1.128(15)$ & $\mathrm{C}(11 \mathrm{~A})-\mathrm{C}(12 \mathrm{~A})$ & $1.43(2)$
\end{tabular}




\begin{tabular}{|c|c|c|c|}
\hline$C(11 A)-C(16 A)$ & $1.47(2)$ & $C(6 A)-C(1 A) \# 5$ & $1.07(2)$ \\
\hline $\mathrm{C}(12 \mathrm{~A})-\mathrm{C}(13)$ & $1.570(14)$ & $\mathrm{C}(6 \mathrm{~A})-\mathrm{N}(1 \mathrm{~A}) \# 5$ & $1.643(18)$ \\
\hline$C(15 A)-C(14)$ & $1.329(15)$ & $\mathrm{C}(6 \mathrm{~A})-\mathrm{C}(5 \mathrm{~A}) \# 5$ & $1.648(15)$ \\
\hline $\mathrm{N}(11)-\mathrm{C}(14)$ & $1.324(11)$ & $\mathrm{Cl}(1)-\mathrm{O}(304)$ & $1.325(13)$ \\
\hline $\mathrm{N}(11)-\mathrm{C}(13)$ & $1.336(10)$ & $\mathrm{Cl}(1)-\mathrm{O}(305)$ & $1.354(8)$ \\
\hline $\mathrm{N}(11)-\mathrm{Cu}(1) \# 4$ & $2.052(5)$ & $\mathrm{Cl}(1)-\mathrm{O}(302)$ & $1.431(9)$ \\
\hline$C(21)-C(22)$ & $1.365(8)$ & $\mathrm{Cl}(1)-\mathrm{O}(301)$ & $1.441(10)$ \\
\hline$C(21)-C(25)$ & $1.384(8)$ & $\mathrm{Cl}(1)-\mathrm{O}(306)$ & $1.450(15)$ \\
\hline$C(21)-C(26)$ & $1.512(8)$ & $\mathrm{O}(301)-\mathrm{O}(304)$ & $1.65(2)$ \\
\hline$C(22)-C(23)$ & $1.368(8)$ & $\mathrm{O}(302)-\mathrm{O}(306)$ & $1.76(2)$ \\
\hline$C(24)-C(25)$ & $1.357(8)$ & $\mathrm{O}(304)-\mathrm{O}(306)$ & $1.59(2)$ \\
\hline$C(31)-C(32)$ & $1.547(10)$ & & \\
\hline $\mathrm{C}(32)-\mathrm{C}(33)$ & $1.520(12)$ & $\mathrm{N}(21) \# 1-\mathrm{Cu}(1)-\mathrm{N}(21)$ & $177.8(3)$ \\
\hline$C(33)-C(34)$ & $1.548(10)$ & $\mathrm{N}(21) \# 1-\mathrm{Cu}(1)-\mathrm{N}(11) \# 2$ & $91.40(18)$ \\
\hline $\mathrm{N}(100)-\mathrm{O}(102)$ & $1.201(9)$ & $\mathrm{N}(21)-\mathrm{Cu}(1)-\mathrm{N}(11) \# 2$ & $88.51(18)$ \\
\hline $\mathrm{N}(100)-\mathrm{O}(101)$ & $1.233(9)$ & $\mathrm{N}(21) \# 1-\mathrm{Cu}(1)-\mathrm{N}(11) \# 3$ & $88.51(18)$ \\
\hline $\mathrm{N}(100)-\mathrm{C}(101)$ & $1.462(10)$ & $\mathrm{N}(21)-\mathrm{Cu}(1)-\mathrm{N}(11) \# 3$ & $91.40(18)$ \\
\hline$C(101)-C(106)$ & $1.378(9)$ & $\mathrm{N}(11) \# 2-\mathrm{Cu}(1)-\mathrm{N}(11) \# 3$ & $175.6(4)$ \\
\hline$C(101)-C(102)$ & $1.398(10)$ & $\mathrm{N}(21) \# 1-\mathrm{Cu}(1)-\mathrm{O}(1 \mathrm{~W})$ & $91.09(14)$ \\
\hline$C(102)-C(103)$ & $1.378(13)$ & $\mathrm{N}(21)-\mathrm{Cu}(1)-\mathrm{O}(1 \mathrm{~W})$ & $91.09(14)$ \\
\hline$C(103)-C(104)$ & $1.385(12)$ & $\mathrm{N}(11) \# 2-\mathrm{Cu}(1)-\mathrm{O}(1 \mathrm{~W})$ & $92.2(2)$ \\
\hline$C(104)-C(105)$ & $1.365(12)$ & $\mathrm{N}(11) \# 3-\mathrm{Cu}(1)-\mathrm{O}(1 \mathrm{~W})$ & $92.2(2)$ \\
\hline$C(105)-C(106)$ & $1.326(11)$ & $\mathrm{C}(23)-\mathrm{N}(21)-\mathrm{C}(24)$ & $116.3(5)$ \\
\hline $\mathrm{N}(1 \mathrm{~A})-\mathrm{C}(2 \mathrm{~A})$ & $0.96(2)$ & $\mathrm{C}(23)-\mathrm{N}(21)-\mathrm{Cu}(1)$ & $122.6(4)$ \\
\hline $\mathrm{N}(1 \mathrm{~A})-\mathrm{C}(3 \mathrm{~A})$ & $1.10(3)$ & $\mathrm{C}(24)-\mathrm{N}(21)-\mathrm{Cu}(1)$ & $121.1(4)$ \\
\hline $\mathrm{N}(1 \mathrm{~A})-\mathrm{C}(1 \mathrm{~A})$ & $1.25(4)$ & $\mathrm{C}(16 \mathrm{~A})-\mathrm{N}(31)-\mathrm{C}(16)$ & $87.0(9)$ \\
\hline $\mathrm{N}(1 \mathrm{~A})-\mathrm{C}(6 \mathrm{~A})$ & $1.643(18)$ & $\mathrm{C}(16 \mathrm{~A})-\mathrm{N}(31)-\mathrm{C}(31)$ & $137.5(12)$ \\
\hline $\mathrm{N}(1 \mathrm{~A})-\mathrm{C}(4 \mathrm{~A})$ & $1.66(3)$ & $\mathrm{C}(16)-\mathrm{N}(31)-\mathrm{C}(31)$ & $135.2(12)$ \\
\hline$C(1 \mathrm{~A})-\mathrm{C}(6 \mathrm{~A})$ & $1.07(2)$ & $\mathrm{C}(26)-\mathrm{N}(32)-\mathrm{C}(34)$ & $121.0(6)$ \\
\hline $\mathrm{C}(1 \mathrm{~A})-\mathrm{C}(5 \mathrm{~A}) \# 5$ & $1.26(4)$ & $\mathrm{C}(15)-\mathrm{C}(11)-\mathrm{C}(12)$ & $119.3(12)$ \\
\hline $\mathrm{C}(1 \mathrm{~A})-\mathrm{C}(2 \mathrm{~A})$ & $1.63(4)$ & $\mathrm{C}(15)-\mathrm{C}(11)-\mathrm{C}(16)$ & $122.0(14)$ \\
\hline $\mathrm{C}(2 \mathrm{~A})-\mathrm{C}(3 \mathrm{~A})$ & $1.55(2)$ & $\mathrm{C}(12)-\mathrm{C}(11)-\mathrm{C}(16)$ & $118.2(13)$ \\
\hline $\mathrm{C}(3 \mathrm{~A})-\mathrm{C}(4 \mathrm{~A})$ & $1.02(2)$ & $\mathrm{C}(13)-\mathrm{C}(12)-\mathrm{C}(11)$ & $108.2(13)$ \\
\hline $\mathrm{C}(4 \mathrm{~A})-\mathrm{C}(5 \mathrm{~A})$ & $1.46(2)$ & $\mathrm{C}(11)-\mathrm{C}(15)-\mathrm{C}(14)$ & $127.4(13)$ \\
\hline $\mathrm{C}(5 \mathrm{~A})-\mathrm{C}(1 \mathrm{~A}) \# 5$ & $1.26(4)$ & $\mathrm{N}(31)-\mathrm{C}(16)-\mathrm{O}(16)$ & $113.3(13)$ \\
\hline$C(5 A)-C(6 A)$ & $1.648(15)$ & $\mathrm{N}(31)-\mathrm{C}(16)-\mathrm{C}(11)$ & $123.9(13)$ \\
\hline
\end{tabular}




\begin{tabular}{|c|c|c|c|}
\hline $\mathrm{O}(16)-\mathrm{C}(16)-\mathrm{C}(11)$ & $122.8(13)$ & $\mathrm{C}(106)-\mathrm{C}(101)-\mathrm{N}(100)$ & $122.3(7)$ \\
\hline$C(15 A)-C(11 A)-C(12 A)$ & $115.6(15)$ & $\mathrm{C}(102)-\mathrm{C}(101)-\mathrm{N}(100)$ & $117.1(7)$ \\
\hline$C(15 A)-C(11 A)-C(16 A)$ & $120.6(13)$ & $\mathrm{C}(103)-\mathrm{C}(102)-\mathrm{C}(101)$ & $118.1(8)$ \\
\hline$C(12 A)-C(11 A)-C(16 A)$ & $123.6(14)$ & $\mathrm{C}(102)-\mathrm{C}(103)-\mathrm{C}(104)$ & $119.6(9)$ \\
\hline $\mathrm{C}(11 \mathrm{~A})-\mathrm{C}(12 \mathrm{~A})-\mathrm{C}(13)$ & $127.1(13)$ & $\mathrm{C}(105)-\mathrm{C}(104)-\mathrm{C}(103)$ & $120.5(9)$ \\
\hline$C(14)-C(15 A)-C(11 A)$ & $112.3(16)$ & $\mathrm{C}(106)-\mathrm{C}(105)-\mathrm{C}(104)$ & $120.9(8)$ \\
\hline $\mathrm{N}(31)-\mathrm{C}(16 \mathrm{~A})-\mathrm{O}(16 \mathrm{~A})$ & $113.3(14)$ & $\mathrm{C}(105)-\mathrm{C}(106)-\mathrm{C}(101)$ & $120.3(8)$ \\
\hline$N(31)-C(16 A)-C(11 A)$ & $128.6(12)$ & $C(2 A)-N(1 A)-C(3 A)$ & $97(2)$ \\
\hline $\mathrm{O}(16 \mathrm{~A})-\mathrm{C}(16 \mathrm{~A})-\mathrm{C}(11 \mathrm{~A})$ & $118.1(13)$ & $\mathrm{C}(2 \mathrm{~A})-\mathrm{N}(1 \mathrm{~A})-\mathrm{C}(1 \mathrm{~A})$ & $94(3)$ \\
\hline $\mathrm{C}(14)-\mathrm{N}(11)-\mathrm{C}(13)$ & $119.4(7)$ & $\mathrm{C}(3 \mathrm{~A})-\mathrm{N}(1 \mathrm{~A})-\mathrm{C}(1 \mathrm{~A})$ & $162(2)$ \\
\hline $\mathrm{C}(14)-\mathrm{N}(11)-\mathrm{Cu}(1) \# 4$ & $120.1(6)$ & $C(2 A)-N(1 A)-C(6 A)$ & $134(3)$ \\
\hline $\mathrm{C}(13)-\mathrm{N}(11)-\mathrm{Cu}(1) \# 4$ & $120.5(5)$ & $C(3 A)-N(1 A)-C(6 A)$ & $128(2)$ \\
\hline $\mathrm{N}(11)-\mathrm{C}(13)-\mathrm{C}(12)$ & $137.6(11)$ & $C(1 \mathrm{~A})-\mathrm{N}(1 \mathrm{~A})-\mathrm{C}(6 \mathrm{~A})$ & $40.9(11)$ \\
\hline $\mathrm{N}(11)-\mathrm{C}(13)-\mathrm{C}(12 \mathrm{~A})$ & $107.8(9)$ & $\mathrm{C}(2 \mathrm{~A})-\mathrm{N}(1 \mathrm{~A})-\mathrm{C}(4 \mathrm{~A})$ & $134(2)$ \\
\hline$C(12)-C(13)-C(12 A)$ & $31.4(7)$ & $\mathrm{C}(3 \mathrm{~A})-\mathrm{N}(1 \mathrm{~A})-\mathrm{C}(4 \mathrm{~A})$ & $37.1(16)$ \\
\hline $\mathrm{N}(11)-\mathrm{C}(14)-\mathrm{C}(15 \mathrm{~A})$ & $136.9(12)$ & $\mathrm{C}(1 \mathrm{~A})-\mathrm{N}(1 \mathrm{~A})-\mathrm{C}(4 \mathrm{~A})$ & $130.2(18)$ \\
\hline $\mathrm{N}(11)-\mathrm{C}(14)-\mathrm{C}(15)$ & $107.3(10)$ & $\mathrm{C}(6 \mathrm{~A})-\mathrm{N}(1 \mathrm{~A})-\mathrm{C}(4 \mathrm{~A})$ & $91.3(12)$ \\
\hline $\mathrm{C}(15 \mathrm{~A})-\mathrm{C}(14)-\mathrm{C}(15)$ & $34.2(10)$ & $\mathrm{C}(6 \mathrm{~A})-\mathrm{C}(1 \mathrm{~A})-\mathrm{N}(1 \mathrm{~A})$ & $89(2)$ \\
\hline$C(22)-C(21)-C(25)$ & $117.2(5)$ & $\mathrm{C}(6 \mathrm{~A})-\mathrm{C}(1 \mathrm{~A})-\mathrm{C}(5 \mathrm{~A}) \# 5$ & $89(3)$ \\
\hline$C(22)-C(21)-C(26)$ & $123.8(5)$ & $\mathrm{N}(1 \mathrm{~A})-\mathrm{C}(1 \mathrm{~A})-\mathrm{C}(5 \mathrm{~A}) \# 5$ & $161(2)$ \\
\hline $\mathrm{C}(25)-\mathrm{C}(21)-\mathrm{C}(26)$ & $119.0(5)$ & $\mathrm{C}(6 \mathrm{~A})-\mathrm{C}(1 \mathrm{~A})-\mathrm{C}(2 \mathrm{~A})$ & $125(3)$ \\
\hline $\mathrm{C}(21)-\mathrm{C}(22)-\mathrm{C}(23)$ & $120.6(6)$ & $\mathrm{N}(1 \mathrm{~A})-\mathrm{C}(1 \mathrm{~A})-\mathrm{C}(2 \mathrm{~A})$ & $36.0(17)$ \\
\hline $\mathrm{N}(21)-\mathrm{C}(23)-\mathrm{C}(22)$ & $123.0(6)$ & $\mathrm{C}(5 \mathrm{~A}) \# 5-\mathrm{C}(1 \mathrm{~A})-\mathrm{C}(2 \mathrm{~A})$ & $138.9(16)$ \\
\hline $\mathrm{C}(25)-\mathrm{C}(24)-\mathrm{N}(21)$ & $123.2(5)$ & $\mathrm{N}(1 \mathrm{~A})-\mathrm{C}(2 \mathrm{~A})-\mathrm{C}(3 \mathrm{~A})$ & $45.0(19)$ \\
\hline $\mathrm{C}(24)-\mathrm{C}(25)-\mathrm{C}(21)$ & 119.6(6) & $\mathrm{N}(1 \mathrm{~A})-\mathrm{C}(2 \mathrm{~A})-\mathrm{C}(1 \mathrm{~A})$ & $50.2(17)$ \\
\hline $\mathrm{O}(26)-\mathrm{C}(26)-\mathrm{N}(32)$ & $122.8(5)$ & $\mathrm{C}(3 \mathrm{~A})-\mathrm{C}(2 \mathrm{~A})-\mathrm{C}(1 \mathrm{~A})$ & $94.2(16)$ \\
\hline $\mathrm{O}(26)-\mathrm{C}(26)-\mathrm{C}(21)$ & $119.9(6)$ & $\mathrm{C}(4 \mathrm{~A})-\mathrm{C}(3 \mathrm{~A})-\mathrm{N}(1 \mathrm{~A})$ & $102(3)$ \\
\hline $\mathrm{N}(32)-\mathrm{C}(26)-\mathrm{C}(21)$ & $117.3(6)$ & $\mathrm{C}(4 \mathrm{~A})-\mathrm{C}(3 \mathrm{~A})-\mathrm{C}(2 \mathrm{~A})$ & $140(3)$ \\
\hline $\mathrm{N}(31)-\mathrm{C}(31)-\mathrm{C}(32)$ & $111.3(5)$ & $\mathrm{N}(1 \mathrm{~A})-\mathrm{C}(3 \mathrm{~A})-\mathrm{C}(2 \mathrm{~A})$ & $38.0(12)$ \\
\hline $\mathrm{C}(33)-\mathrm{C}(32)-\mathrm{C}(31)$ & $113.7(7)$ & $\mathrm{C}(3 \mathrm{~A})-\mathrm{C}(4 \mathrm{~A})-\mathrm{C}(5 \mathrm{~A})$ & $128(2)$ \\
\hline $\mathrm{C}(32)-\mathrm{C}(33)-\mathrm{C}(34)$ & 109.0(7) & $\mathrm{C}(3 \mathrm{~A})-\mathrm{C}(4 \mathrm{~A})-\mathrm{N}(1 \mathrm{~A})$ & $40.7(18)$ \\
\hline $\mathrm{N}(32)-\mathrm{C}(34)-\mathrm{C}(33)$ & $109.4(5)$ & $\mathrm{C}(5 \mathrm{~A})-\mathrm{C}(4 \mathrm{~A})-\mathrm{N}(1 \mathrm{~A})$ & $87.8(10)$ \\
\hline $\mathrm{O}(102)-\mathrm{N}(100)-\mathrm{O}(101)$ & $122.9(9)$ & $\mathrm{C}(1 \mathrm{~A}) \# 5-\mathrm{C}(5 \mathrm{~A})-\mathrm{C}(4 \mathrm{~A})$ & $138.1(18)$ \\
\hline $\mathrm{O}(102)-\mathrm{N}(100)-\mathrm{C}(101)$ & $120.2(8)$ & $\mathrm{C}(1 \mathrm{~A}) \# 5-\mathrm{C}(5 \mathrm{~A})-\mathrm{C}(6 \mathrm{~A})$ & $40.7(11)$ \\
\hline $\mathrm{O}(101)-\mathrm{N}(100)-\mathrm{C}(101)$ & $116.6(8)$ & $\mathrm{C}(4 \mathrm{~A})-\mathrm{C}(5 \mathrm{~A})-\mathrm{C}(6 \mathrm{~A})$ & $98.7(12)$ \\
\hline $\mathrm{C}(106)-\mathrm{C}(101)-\mathrm{C}(102)$ & $120.6(8)$ & $\mathrm{C}(1 \mathrm{~A}) \# 5-\mathrm{C}(6 \mathrm{~A})-\mathrm{C}(1 \mathrm{~A})$ & $180(5)$ \\
\hline
\end{tabular}




$\begin{array}{lclc}\mathrm{C}(1 \mathrm{~A}) \# 5-\mathrm{C}(6 \mathrm{~A})-\mathrm{N}(1 \mathrm{~A}) & 130(2) & \mathrm{O}(305)-\mathrm{Cl}(1)-\mathrm{O}(302) & 119.6(7) \\ \mathrm{C}(1 \mathrm{~A})-\mathrm{C}(6 \mathrm{~A})-\mathrm{N}(1 \mathrm{~A}) & 50(2) & \mathrm{O}(304)-\mathrm{Cl}(1)-\mathrm{O}(301) & 73.2(11) \\ \mathrm{C}(1 \mathrm{~A}) \# 5-\mathrm{C}(6 \mathrm{~A})-\mathrm{N}(1 \mathrm{~A}) \# 5 & 50(2) & \mathrm{O}(305)-\mathrm{Cl}(1)-\mathrm{O}(301) & 110.0(7) \\ \mathrm{C}(1 \mathrm{~A})-\mathrm{C}(6 \mathrm{~A})-\mathrm{N}(1 \mathrm{~A}) \# 5 & 130(2) & \mathrm{O}(302)-\mathrm{Cl}(1)-\mathrm{O}(301) & 106.6(7) \\ \mathrm{N}(1 \mathrm{~A})-\mathrm{C}(6 \mathrm{~A})-\mathrm{N}(1 \mathrm{~A}) \# 5 & 180.000(7) & \mathrm{O}(304)-\mathrm{Cl}(1)-\mathrm{O}(306) & 69.5(11) \\ \mathrm{C}(1 \mathrm{~A}) \# 5-\mathrm{C}(6 \mathrm{~A})-\mathrm{C}(5 \mathrm{~A}) \# 5 & 130(2) & \mathrm{O}(305)-\mathrm{Cl}(1)-\mathrm{O}(306) & 106.2(10) \\ \mathrm{C}(1 \mathrm{~A})-\mathrm{C}(6 \mathrm{~A})-\mathrm{C}(5 \mathrm{~A}) \# 5 & 50(2) & \mathrm{O}(302)-\mathrm{Cl}(1)-\mathrm{O}(306) & 75.3(10) \\ \mathrm{N}(1 \mathrm{~A})-\mathrm{C}(6 \mathrm{~A})-\mathrm{C}(5 \mathrm{~A}) \# 5 & 97.9(12) & \mathrm{O}(301)-\mathrm{Cl}(1)-\mathrm{O}(306) & 135.8(10) \\ \mathrm{N}(1 \mathrm{~A}) \# 5-\mathrm{C}(6 \mathrm{~A})-\mathrm{C}(5 \mathrm{~A}) \# 5 & 82.1(12) & \mathrm{Cl}(1)-\mathrm{O}(301)-\mathrm{O}(304) & 50.2(6) \\ \mathrm{C}(1 \mathrm{~A}) \# 5-\mathrm{C}(6 \mathrm{~A})-\mathrm{C}(5 \mathrm{~A}) & 50(2) & \mathrm{Cl}(1)-\mathrm{O}(302)-\mathrm{O}(306) & 52.8(7) \\ \mathrm{C}(1 \mathrm{~A})-\mathrm{C}(6 \mathrm{~A})-\mathrm{C}(5 \mathrm{~A}) & 130(2) & \mathrm{Cl}(1)-\mathrm{O}(304)-\mathrm{O}(306) & 58.9(8) \\ \mathrm{N}(1 \mathrm{~A})-\mathrm{C}(6 \mathrm{~A})-\mathrm{C}(5 \mathrm{~A}) & 82.1(11) & \mathrm{Cl}(1)-\mathrm{O}(304)-\mathrm{O}(301) & 56.6(8) \\ \mathrm{N}(1 \mathrm{~A}) \# 5-\mathrm{C}(6 \mathrm{~A})-\mathrm{C}(5 \mathrm{~A}) & 97.9(12) & \mathrm{O}(306)-\mathrm{O}(304)-\mathrm{O}(301) & 111.6(11) \\ \mathrm{C}(5 \mathrm{~A}) \# 5-\mathrm{C}(6 \mathrm{~A})-\mathrm{C}(5 \mathrm{~A}) & 180.000(3) & \mathrm{Cl}(1)-\mathrm{O}(306)-\mathrm{O}(304) & 51.5(8) \\ \mathrm{O}(304)-\mathrm{Cl}(1)-\mathrm{O}(305) & 113.6(10) & \mathrm{Cl}(1)-\mathrm{O}(306)-\mathrm{O}(302) & 51.9(7) \\ \mathrm{O}(304)-\mathrm{Cl}(1)-\mathrm{O}(302) & 122.1(10) & \mathrm{O}(304)-\mathrm{O}(306)-\mathrm{O}(302) & 92.1(12)\end{array}$

Symmetry transformations used to generate equivalent atoms:

$\# 1-\mathrm{x}, \mathrm{y},-\mathrm{z}+1 / 2 \quad \# 2-\mathrm{x}-1 / 2,-\mathrm{y}+3 / 2, \mathrm{z}+1 / 2 \quad \# 3 \mathrm{x}+1 / 2,-\mathrm{y}+3 / 2,-\mathrm{z}$

\#4 -x-1/2,-y+3/2,z-1/2 \#5-x,-y+1,-z+1

Table 4. Anisotropic displacement parameters $\left(\AA^{2} \times 10^{3}\right)$ for 2 . The anisotropic displacement factor exponent takes the form: $-2 \pi^{2}\left[h^{2} a^{* 2} U^{11}+\ldots+2 h k a^{*} b^{*} U^{12}\right]$

\begin{tabular}{lcccccc}
\hline & $\mathrm{U}^{11}$ & $\mathrm{U}^{22}$ & $\mathrm{U}^{33}$ & $\mathrm{U}^{23}$ & $\mathrm{U}^{13}$ & $\mathrm{U}^{12}$ \\
\hline $\mathrm{Cu}(1)$ & $33(1)$ & $51(1)$ & $24(1)$ & 0 & $0(1)$ & 0 \\
$\mathrm{O}(1 \mathrm{~W})$ & $90(5)$ & $49(4)$ & $74(5)$ & 0 & $-19(5)$ & 0 \\
$\mathrm{O}(2 \mathrm{~W})$ & $112(7)$ & $37(4)$ & $160(9)$ & 0 & $-62(7)$ & 0 \\
$\mathrm{~N}(21)$ & $42(3)$ & $36(3)$ & $33(3)$ & $-5(2)$ & $3(2)$ & $0(2)$ \\
$\mathrm{O}(26)$ & $51(3)$ & $56(3)$ & $60(3)$ & $2(3)$ & $-6(2)$ & $-18(2)$ \\
$\mathrm{N}(31)$ & $45(4)$ & $161(8)$ & $42(4)$ & $36(5)$ & $-17(3)$ & $-40(5)$ \\
$\mathrm{N}(32)$ & $48(3)$ & $56(3)$ & $33(3)$ & $16(3)$ & $-7(2)$ & $-8(3)$ \\
$\mathrm{O}(16)$ & $52(6)$ & $38(6)$ & $50(5)$ & $8(4)$ & $-18(4)$ & $6(4)$ \\
$\mathrm{C}(11)$ & $40(5)$ & $44(5)$ & $22(5)$ & $6(7)$ & $-29(6)$ & $-15(4)$ \\
$\mathrm{C}(12)$ & $43(7)$ & $38(8)$ & $37(8)$ & $-2(6)$ & $6(6)$ & $-14(7)$ \\
$\mathrm{C}(15)$ & $63(12)$ & $63(12)$ & $18(6)$ & $6(7)$ & $-18(7)$ & $42(11)$
\end{tabular}




\begin{tabular}{|c|c|c|c|c|c|c|}
\hline$C(16)$ & $19(6)$ & $43(8)$ & $44(7)$ & $5(6)$ & $-11(5)$ & $-2(6)$ \\
\hline $\mathrm{O}(16 \mathrm{~A})$ & $56(6)$ & $47(6)$ & $59(6)$ & $-1(4)$ & $-21(5)$ & $10(5)$ \\
\hline$C(11 \mathrm{~A})$ & $44(8)$ & $33(8)$ & $58(9)$ & $1(7)$ & $6(8)$ & $4(8)$ \\
\hline$C(12 A)$ & $40(5)$ & $44(5)$ & $23(5)$ & $6(7)$ & $-29(6)$ & $-14(4)$ \\
\hline$C(15 A)$ & $39(8)$ & $72(11)$ & $44(8)$ & $5(8)$ & $-7(6)$ & $2(8)$ \\
\hline$C(16 A)$ & $62(8)$ & $12(5)$ & $35(7)$ & $-16(5)$ & $11(6)$ & $-8(5)$ \\
\hline $\mathrm{N}(11)$ & $48(3)$ & $88(5)$ & $31(3)$ & $11(3)$ & $-3(2)$ & $-19(3)$ \\
\hline$C(13)$ & $35(4)$ & $126(7)$ & $58(5)$ & $44(5)$ & $-18(3)$ & $-8(4)$ \\
\hline $\mathrm{C}(14)$ & $55(5)$ & 171(11) & $46(4)$ & $-31(6)$ & $-6(4)$ & $-32(6)$ \\
\hline$C(21)$ & $36(3)$ & $43(3)$ & $26(3)$ & $-1(3)$ & $10(2)$ & $2(3)$ \\
\hline $\mathrm{C}(22)$ & $44(3)$ & $46(4)$ & $33(3)$ & $12(3)$ & $-6(3)$ & $-5(3)$ \\
\hline $\mathrm{C}(23)$ & $42(3)$ & $46(4)$ & $33(3)$ & $7(3)$ & $0(3)$ & $-11(3)$ \\
\hline $\mathrm{C}(24)$ & $55(4)$ & $35(3)$ & $41(3)$ & $12(3)$ & $1(3)$ & $-2(3)$ \\
\hline$C(25)$ & $38(3)$ & $36(3)$ & $39(3)$ & $9(3)$ & $-6(3)$ & $-6(3)$ \\
\hline$C(26)$ & $34(3)$ & $50(4)$ & $26(3)$ & $-11(3)$ & $4(2)$ & $-3(3)$ \\
\hline $\mathrm{C}(31)$ & $48(4)$ & $84(5)$ & $43(4)$ & $17(4)$ & $-21(3)$ & $-30(4)$ \\
\hline$C(32)$ & $66(5)$ & $87(6)$ & $40(4)$ & $17(4)$ & $-18(3)$ & $-18(4)$ \\
\hline$C(33)$ & $56(4)$ & $92(6)$ & $43(4)$ & $21(4)$ & $-17(3)$ & $-17(4)$ \\
\hline $\mathrm{C}(34)$ & $54(4)$ & $93(6)$ & $41(4)$ & $28(4)$ & $-15(3)$ & $-28(4)$ \\
\hline $\mathrm{N}(100)$ & $66(5)$ & $70(5)$ & $88(5)$ & $5(4)$ & $6(4)$ & $-13(4)$ \\
\hline $\mathrm{O}(101)$ & $103(5)$ & $132(7)$ & $130(6)$ & $-29(5)$ & $25(5)$ & $25(5)$ \\
\hline $\mathrm{O}(102)$ & $100(5)$ & $114(6)$ & $90(5)$ & $0(4)$ & $-14(4)$ & $-34(5)$ \\
\hline$C(101)$ & $49(4)$ & $57(4)$ & $56(4)$ & $11(4)$ & $6(3)$ & $-5(3)$ \\
\hline$C(102)$ & $53(4)$ & $91(6)$ & $63(5)$ & $30(5)$ & $8(4)$ & $2(5)$ \\
\hline$C(103)$ & $71(6)$ & 109(8) & $79(6)$ & $27(6)$ & $19(5)$ & $32(6)$ \\
\hline C(104) & $107(8)$ & $76(6)$ & $47(4)$ & $11(4)$ & $6(5)$ & $3(6)$ \\
\hline$C(105)$ & $63(5)$ & $87(6)$ & $60(5)$ & $6(5)$ & $2(4)$ & $-11(5)$ \\
\hline$C(106)$ & $47(4)$ & $67(5)$ & $61(4)$ & $23(4)$ & $0(3)$ & $11(4)$ \\
\hline $\mathrm{N}(1 \mathrm{~A})$ & $124(17)$ & $75(12)$ & $52(10)$ & $22(9)$ & $9(12)$ & $42(12)$ \\
\hline $\mathrm{C}(1 \mathrm{~A})$ & $62(12)$ & $32(9)$ & $240(40)$ & $64(16)$ & $-54(19)$ & $-23(9)$ \\
\hline $\mathrm{C}(2 \mathrm{~A})$ & 121(11) & $168(15)$ & $77(8)$ & $8(9)$ & $28(8)$ & $17(11)$ \\
\hline $\mathrm{C}(3 \mathrm{~A})$ & $65(11)$ & $76(13)$ & $87(16)$ & $29(11)$ & $-45(11)$ & $-34(10)$ \\
\hline $\mathrm{C}(4 \mathrm{~A})$ & $156(13)$ & $136(12)$ & $77(7)$ & $-31(8)$ & $-38(8)$ & $90(10)$ \\
\hline $\mathrm{C}(5 \mathrm{~A})$ & $67(11)$ & $58(10)$ & $86(13)$ & $-38(10)$ & $-8(9)$ & $-21(9)$ \\
\hline$C(6 A)$ & $116(14)$ & 137(16) & $81(10)$ & $-29(11)$ & $-38(12)$ & $69(15)$ \\
\hline $\mathrm{Cl}(1)$ & $50(1)$ & $79(1)$ & $94(2)$ & $-14(1)$ & $-6(1)$ & 1(1) \\
\hline
\end{tabular}




\begin{tabular}{ccccccc}
$\mathrm{O}(301)$ & $190(13)$ & $134(9)$ & $137(9)$ & $8(8)$ & $-48(9)$ & $87(9)$ \\
$\mathrm{O}(302)$ & $158(9)$ & $136(9)$ & $152(9)$ & $-20(7)$ & $9(8)$ & $83(8)$ \\
$\mathrm{O}(304)$ & $85(8)$ & $310(20)$ & $207(17)$ & $-63(18)$ & $8(9)$ & $6(12)$ \\
$\mathrm{O}(305)$ & $251(12)$ & $89(6)$ & $253(12)$ & $-61(7)$ & $-180(11)$ & $37(7)$ \\
$\mathrm{O}(306)$ & $210(20)$ & $102(12)$ & $240(20)$ & $-11(13)$ & $-25(18)$ & $-83(13)$ \\
\hline
\end{tabular}

Table 5. Hydrogen coordinates ( $\left.\mathrm{x} 10^{4}\right)$ and isotropic displacement parameters $\left(\AA^{2} \mathrm{x} 10^{3}\right)$ For 2.

\begin{tabular}{|c|c|c|c|c|}
\hline & $\mathrm{x}$ & $\mathrm{y}$ & $\mathrm{z}$ & $\mathrm{U}(\mathrm{eq})$ \\
\hline $\mathrm{H}(32)$ & -2470 & 8414 & 946 & 55 \\
\hline $\mathrm{H}(12)$ & -2795 & 6376 & -1825 & 47 \\
\hline $\mathrm{H}(15)$ & -3633 & 9387 & -1130 & 57 \\
\hline $\mathrm{H}(12 \mathrm{~A})$ & -2725 & 7284 & -1633 & 43 \\
\hline $\mathrm{H}(15 \mathrm{~A})$ & -3889 & 10493 & -1266 & 62 \\
\hline $\mathrm{H}(13)$ & -3683 & 6875 & -2282 & 88 \\
\hline $\mathrm{H}(14)$ & -4560 & 9944 & -1711 & 109 \\
\hline $\mathrm{H}(22)$ & -1428 & 8398 & 1238 & 49 \\
\hline $\mathrm{H}(23)$ & -520 & 8390 & 1734 & 48 \\
\hline $\mathrm{H}(24)$ & -1263 & 5071 & 2244 & 52 \\
\hline $\mathrm{H}(25)$ & -2138 & 4941 & 1731 & 45 \\
\hline $\mathrm{H}(31 \mathrm{~A})$ & -1782 & 9516 & -393 & 70 \\
\hline $\mathrm{H}(31 \mathrm{~B})$ & -1419 & 8178 & -554 & 70 \\
\hline $\mathrm{H}(32 \mathrm{~A})$ & -2304 & 6922 & -156 & 77 \\
\hline $\mathrm{H}(32 \mathrm{~B})$ & -1811 & 7780 & 151 & 77 \\
\hline $\mathrm{H}(33 \mathrm{~A})$ & -3210 & 8612 & -99 & 76 \\
\hline $\mathrm{H}(33 \mathrm{~B})$ & -2721 & 9382 & 235 & 76 \\
\hline $\mathrm{H}(34 \mathrm{~A})$ & -3611 & 7923 & 583 & 75 \\
\hline $\mathrm{H}(34 \mathrm{~B})$ & -3189 & 6684 & 394 & 75 \\
\hline $\mathrm{H}(102)$ & -864 & 10310 & 701 & 83 \\
\hline $\mathrm{H}(103)$ & -876 & 11693 & 1310 & 104 \\
\hline $\mathrm{H}(104)$ & 134 & 11806 & 1750 & 92 \\
\hline $\mathrm{H}(105)$ & 1119 & 10501 & 1605 & 84 \\
\hline $\mathrm{H}(106)$ & 1146 & 9156 & 1018 & 70 \\
\hline
\end{tabular}


Table 1. Crystal data and structure refinement for $\mathbf{3}$.

Identification code

Empirical formula

Formula weight

Temperature

Wavelength

Crystal system

Space group

Unit cell dimensions

Volume

Z

Density (calculated)

Absorption coefficient

$\mathrm{F}(000)$

Crystal size

Theta range for data collection

Index ranges

Reflections collected

Independent reflections

Completeness to theta $=24.96^{\circ}$

Absorption correction

Refinement method

Data / restraints / parameters

Goodness-of-fit on $\mathrm{F}^{2}$

Final $\mathrm{R}$ indices [I $>2 \operatorname{sigma}(\mathrm{I})]$

$\mathrm{R}$ indices (all data)

Absolute structure parameter

Largest diff. peak and hole
3

C34 H48 Cl2 Cu N8 O16

959.24

293(2) K

$0.71073 \AA$

Trigonal

P31

$\mathrm{a}=17.943(3) \AA \quad \alpha=90^{\circ}$.

$\mathrm{b}=17.943(3) \AA \quad \beta=90^{\circ}$.

$\mathrm{c}=13.252(3) \AA \quad \gamma=120^{\circ}$.

3694.9(10) $\AA^{3}$

3

$1.293 \mathrm{Mg} / \mathrm{m}^{3}$

$0.620 \mathrm{~mm}^{-1}$

1497

$0.15 \times 0.15 \times 0.10 \mathrm{~mm}^{3}$

1.31 to $24.96^{\circ}$.

$-18<=\mathrm{h}<=21,-18<=\mathrm{k}<=21,-15<=\mathrm{l}<=15$

4699

$4684[\mathrm{R}($ int $)=0.0122]$

$98.0 \%$

None

Full-matrix least-squares on $\mathrm{F}^{2}$

4684 / 1 / 532

1.288

$\mathrm{R} 1=0.0767, \mathrm{wR} 2=0.1895$

$\mathrm{R} 1=0.0940, \mathrm{wR} 2=0.2122$

$0.09(3)$

0.486 and -0.424 e. $\AA^{-3}$ 
Table 2. Atomic coordinates ( $\times 10^{4}$ ) and equivalent isotropic displacement parameters $\left(\AA^{2} \times 10^{3}\right)$ for 3. $U(e q)$ is defined as one third of the trace of the orthogonalized $U^{\mathrm{ij}}$ tensor.

\begin{tabular}{|c|c|c|c|c|}
\hline & $\mathrm{x}$ & $\mathrm{y}$ & $\mathrm{z}$ & $\mathrm{U}(\mathrm{eq})$ \\
\hline $\mathrm{Cu}(1)$ & $7367(1)$ & $20669(1)$ & $3506(1)$ & $35(1)$ \\
\hline $\mathrm{O}(1 \mathrm{~W})$ & $3305(5)$ & 2618(5) & $2055(7)$ & $54(2)$ \\
\hline $\mathrm{O}(2 \mathrm{~W})$ & $7267(6)$ & $20566(6)$ & $1715(6)$ & $56(2)$ \\
\hline $\mathrm{N}(11 \mathrm{~A})$ & 6992(7) & 19397(6) & $3588(8)$ & $49(2)$ \\
\hline $\mathrm{O}(16 \mathrm{~A})$ & $6541(9)$ & $16712(7)$ & $5247(9)$ & $94(4)$ \\
\hline $\mathrm{C}(11 \mathrm{~A})$ & $6671(8)$ & $17719(6)$ & $3967(10)$ & $53(3)$ \\
\hline $\mathrm{C}(12 \mathrm{~A})$ & $6317(8)$ & $18109(8)$ & $4611(11)$ & $61(3)$ \\
\hline $\mathrm{C}(13 \mathrm{~A})$ & $6488(7)$ & $18915(7)$ & 4411(9) & $49(3)$ \\
\hline $\mathrm{C}(14 \mathrm{~A})$ & $7320(8)$ & 19048(8) & 2963(9) & $49(3)$ \\
\hline$C(15 A)$ & $7108(8)$ & $18169(7)$ & $3157(10)$ & $53(3)$ \\
\hline $\mathrm{C}(16 \mathrm{~A})$ & $6535(11)$ & 16851(8) & $4275(10)$ & $69(4)$ \\
\hline $\mathrm{N}(21 \mathrm{~A})$ & $7747(5)$ & $11954(5)$ & $3485(7)$ & $42(2)$ \\
\hline $\mathrm{O}(26 \mathrm{~A})$ & 8891(6) & $15080(5)$ & $4172(7)$ & $60(2)$ \\
\hline $\mathrm{C}(21 \mathrm{~A})$ & $8237(9)$ & $13666(7)$ & $3469(10)$ & $58(3)$ \\
\hline $\mathrm{C}(22 \mathrm{~A})$ & $8608(7)$ & $13365(6)$ & $4095(8)$ & $43(2)$ \\
\hline$C(23 \mathrm{~A})$ & $8362(7)$ & $12534(6)$ & $4118(8)$ & $44(3)$ \\
\hline$C(24 \mathrm{~A})$ & $7338(7)$ & $12236(6)$ & $2880(8)$ & $42(2)$ \\
\hline$C(25 \mathrm{~A})$ & 7597(8) & $13106(7)$ & $2832(8)$ & $47(2)$ \\
\hline$C(26 \mathrm{~A})$ & $8535(8)$ & $14645(7)$ & $3474(9)$ & $50(3)$ \\
\hline $\mathrm{N}(31 \mathrm{~A})$ & $6410(8)$ & $16314(7)$ & $3551(9)$ & $70(3)$ \\
\hline $\mathrm{N}(32 \mathrm{~A})$ & $8340(8)$ & $14930(7)$ & 2613(8) & $68(3)$ \\
\hline $\mathrm{C}(31 \mathrm{~A})$ & $6363(10)$ & $15462(7)$ & 3801(13) & $72(4)$ \\
\hline $\mathrm{C}(32 \mathrm{~A})$ & $7322(10)$ & 15598(9) & $3647(12)$ & $70(4)$ \\
\hline$C(33 \mathrm{~A})$ & $7641(10)$ & 15791(8) & $2668(10)$ & $63(3)$ \\
\hline $\mathrm{C}(34 \mathrm{~A})$ & $8469(10)$ & $15787(9)$ & $2522(10)$ & $65(3)$ \\
\hline $\mathrm{N}(11 \mathrm{~B})$ & $6091(5)$ & $20323(5)$ & $3527(7)$ & $41(2)$ \\
\hline $\mathrm{O}(16 \mathrm{~B})$ & $3274(6)$ & 19748(8) & 4735(9) & $83(3)$ \\
\hline$C(11 B)$ & 4371(8) & 19809(9) & $3733(10)$ & $54(3)$ \\
\hline$C(12 B)$ & 4980(7) & $20473(7)$ & $4295(10)$ & $52(3)$ \\
\hline$C(13 B)$ & $5828(8)$ & $20710(8)$ & $4206(11)$ & $58(3)$ \\
\hline$C(14 B)$ & $5540(6)$ & $19756(7)$ & 2988(9) & $43(3)$ \\
\hline
\end{tabular}




\begin{tabular}{|c|c|c|c|c|}
\hline$C(15 B)$ & $4684(8)$ & 19444(9) & $3026(12)$ & $72(4)$ \\
\hline$C(16 B)$ & $3470(8)$ & $19566(8)$ & 3951(10) & $55(3)$ \\
\hline $\mathrm{N}(21 \mathrm{~B})$ & $-1399(5)$ & $20938(6)$ & $3524(6)$ & $37(2)$ \\
\hline $\mathrm{O}(26 \mathrm{~B})$ & $1787(5)$ & $22033(6)$ & $3149(8)$ & $68(3)$ \\
\hline$C(21 B)$ & 293(7) & $21218(7)$ & $3558(7)$ & $40(2)$ \\
\hline$C(22 B)$ & $-356(8)$ & 20616(8) & 4182(8) & $51(3)$ \\
\hline$C(23 B)$ & $-1150(7)$ & $20510(7)$ & $4178(7)$ & $40(2)$ \\
\hline$C(24 B)$ & $-767(8)$ & $21507(8)$ & 2885(9) & $54(3)$ \\
\hline$C(25 B)$ & $55(9)$ & $21648(7)$ & 2892(9) & $57(3)$ \\
\hline$C(26 B)$ & 1211(6) & $21356(8)$ & $3501(8)$ & $47(3)$ \\
\hline $\mathrm{N}(31 \mathrm{~B})$ & 2898(7) & 19143(9) & $3181(8)$ & $72(3)$ \\
\hline $\mathrm{N}(32 \mathrm{~B})$ & 1303(6) & $20695(7)$ & $3824(9)$ & $57(3)$ \\
\hline$C(31 B)$ & 2018(7) & $18980(10)$ & $3215(11)$ & $66(4)$ \\
\hline$C(32 B)$ & 1934(11) & 19611(12) & $2618(13)$ & $83(5)$ \\
\hline$C(33 B)$ & $2451(12)$ & 20561(14) & $2965(14)$ & $95(6)$ \\
\hline$C(34 B)$ & 2171(8) & $20787(10)$ & $3915(10)$ & $64(3)$ \\
\hline $\mathrm{Cl}(1)$ & $8900(2)$ & $487(2)$ & $204(3)$ & $64(1)$ \\
\hline $\mathrm{O}(101)$ & 8112(9) & 189(11) & $-385(17)$ & $158(8)$ \\
\hline $\mathrm{O}(102)$ & $9182(14)$ & 1337(8) & $479(14)$ & $150(7)$ \\
\hline $\mathrm{O}(103)$ & $9446(10)$ & 431(11) & $-483(8)$ & $110(5)$ \\
\hline $\mathrm{O}(104)$ & $8848(9)$ & $-31(8)$ & 1060(9) & $99(4)$ \\
\hline $\mathrm{Cl}(2)$ & $5340(2)$ & $12888(2)$ & $3247(2)$ & $64(1)$ \\
\hline $\mathrm{O}(201)$ & $5748(11)$ & $13355(12)$ & $2362(10)$ & $139(6)$ \\
\hline $\mathrm{O}(202)$ & $5867(9)$ & $13205(13)$ & 4107(8) & $128(6)$ \\
\hline $\mathrm{O}(203)$ & $4920(20)$ & $12087(10)$ & $3244(17)$ & 273(19) \\
\hline $\mathrm{O}(204)$ & $4708(11)$ & 13133(17) & $3302(13)$ & $169(8)$ \\
\hline $\mathrm{O}(3 \mathrm{~W})$ & $1364(18)$ & 7633(19) & $490(20)$ & $209(10)$ \\
\hline $\mathrm{O}(4 \mathrm{~W})$ & $5780(30)$ & $16870(20)$ & $7570(30)$ & $370(30)$ \\
\hline
\end{tabular}


Table 3. Bond lengths $[\AA]$ and angles $\left[{ }^{\circ}\right]$ for 3 .

\begin{tabular}{|c|c|c|c|}
\hline $\mathrm{Cu}(1)-\mathrm{N}(21 \mathrm{~B}) \# 1$ & $2.018(8)$ & $\mathrm{C}(11 \mathrm{~B})-\mathrm{C}(16 \mathrm{~B})$ & $1.477(16)$ \\
\hline $\mathrm{Cu}(1)-\mathrm{N}(11 \mathrm{~A})$ & $2.035(9)$ & $\mathrm{C}(12 \mathrm{~B})-\mathrm{C}(13 \mathrm{~B})$ & $1.365(16)$ \\
\hline $\mathrm{Cu}(1)-\mathrm{N}(11 \mathrm{~B})$ & $2.050(8)$ & $\mathrm{C}(14 \mathrm{~B})-\mathrm{C}(15 \mathrm{~B})$ & $1.348(16)$ \\
\hline $\mathrm{Cu}(1)-\mathrm{N}(21 \mathrm{~A}) \# 2$ & $2.051(8)$ & $\mathrm{C}(16 \mathrm{~B})-\mathrm{N}(31 \mathrm{~B})$ & $1.375(18)$ \\
\hline $\mathrm{Cu}(1)-\mathrm{O}(2 \mathrm{~W})$ & $2.380(8)$ & $\mathrm{N}(21 \mathrm{~B})-\mathrm{C}(24 \mathrm{~B})$ & $1.374(14)$ \\
\hline $\mathrm{N}(11 \mathrm{~A})-\mathrm{C}(14 \mathrm{~A})$ & $1.340(14)$ & $\mathrm{N}(21 \mathrm{~B})-\mathrm{C}(23 \mathrm{~B})$ & $1.372(13)$ \\
\hline $\mathrm{N}(11 \mathrm{~A})-\mathrm{C}(13 \mathrm{~A})$ & $1.404(15)$ & $\mathrm{N}(21 \mathrm{~B})-\mathrm{Cu}(1) \# 4$ & $2.018(8)$ \\
\hline $\mathrm{O}(16 \mathrm{~A})-\mathrm{C}(16 \mathrm{~A})$ & $1.313(17)$ & $\mathrm{O}(26 \mathrm{~B})-\mathrm{C}(26 \mathrm{~B})$ & $1.228(15)$ \\
\hline $\mathrm{C}(11 \mathrm{~A})-\mathrm{C}(15 \mathrm{~A})$ & $1.336(18)$ & $\mathrm{C}(21 \mathrm{~B})-\mathrm{C}(25 \mathrm{~B})$ & $1.374(16)$ \\
\hline $\mathrm{C}(11 \mathrm{~A})-\mathrm{C}(12 \mathrm{~A})$ & $1.437(17)$ & $\mathrm{C}(21 \mathrm{~B})-\mathrm{C}(22 \mathrm{~B})$ & $1.396(16)$ \\
\hline$C(11 A)-C(16 A)$ & $1.509(15)$ & $\mathrm{C}(21 \mathrm{~B})-\mathrm{C}(26 \mathrm{~B})$ & $1.539(14)$ \\
\hline $\mathrm{C}(12 \mathrm{~A})-\mathrm{C}(13 \mathrm{~A})$ & $1.347(16)$ & $\mathrm{C}(22 \mathrm{~B})-\mathrm{C}(23 \mathrm{~B})$ & $1.340(16)$ \\
\hline $\mathrm{C}(14 \mathrm{~A})-\mathrm{C}(15 \mathrm{~A})$ & $1.449(15)$ & $\mathrm{C}(24 \mathrm{~B})-\mathrm{C}(25 \mathrm{~B})$ & $1.367(18)$ \\
\hline$C(16 A)-N(31 A)$ & $1.297(17)$ & $\mathrm{C}(26 \mathrm{~B})-\mathrm{N}(32 \mathrm{~B})$ & $1.346(16)$ \\
\hline $\mathrm{N}(21 \mathrm{~A})-\mathrm{C}(24 \mathrm{~A})$ & $1.345(13)$ & $\mathrm{N}(31 \mathrm{~B})-\mathrm{C}(31 \mathrm{~B})$ & $1.455(15)$ \\
\hline $\mathrm{N}(21 \mathrm{~A})-\mathrm{C}(23 \mathrm{~A})$ & $1.363(13)$ & $\mathrm{N}(32 \mathrm{~B})-\mathrm{C}(34 \mathrm{~B})$ & $1.486(16)$ \\
\hline $\mathrm{N}(21 \mathrm{~A})-\mathrm{Cu}(1) \# 3$ & $2.051(8)$ & $\mathrm{C}(31 \mathrm{~B})-\mathrm{C}(32 \mathrm{~B})$ & $1.45(2)$ \\
\hline $\mathrm{O}(26 \mathrm{~A})-\mathrm{C}(26 \mathrm{~A})$ & $1.173(15)$ & $\mathrm{C}(32 \mathrm{~B})-\mathrm{C}(33 \mathrm{~B})$ & $1.55(3)$ \\
\hline$C(21 \mathrm{~A})-\mathrm{C}(22 \mathrm{~A})$ & $1.335(17)$ & $\mathrm{C}(33 \mathrm{~B})-\mathrm{C}(34 \mathrm{~B})$ & $1.49(2)$ \\
\hline$C(21 \mathrm{~A})-\mathrm{C}(25 \mathrm{~A})$ & $1.374(16)$ & $\mathrm{Cl}(1)-\mathrm{O}(103)$ & $1.378(10)$ \\
\hline$C(21 A)-C(26 A)$ & $1.560(15)$ & $\mathrm{Cl}(1)-\mathrm{O}(102)$ & $1.394(11)$ \\
\hline$C(22 \mathrm{~A})-\mathrm{C}(23 \mathrm{~A})$ & $1.326(14)$ & $\mathrm{Cl}(1)-\mathrm{O}(104)$ & $1.439(12)$ \\
\hline$C(24 \mathrm{~A})-\mathrm{C}(25 \mathrm{~A})$ & $1.389(15)$ & $\mathrm{Cl}(1)-\mathrm{O}(101)$ & $1.463(13)$ \\
\hline $\mathrm{C}(26 \mathrm{~A})-\mathrm{N}(32 \mathrm{~A})$ & $1.365(16)$ & $\mathrm{Cl}(2)-\mathrm{O}(203)$ & $1.246(17)$ \\
\hline $\mathrm{N}(31 \mathrm{~A})-\mathrm{C}(31 \mathrm{~A})$ & $1.524(16)$ & $\mathrm{Cl}(2)-\mathrm{O}(204)$ & $1.41(2)$ \\
\hline $\mathrm{N}(32 \mathrm{~A})-\mathrm{C}(34 \mathrm{~A})$ & $1.441(16)$ & $\mathrm{Cl}(2)-\mathrm{O}(202)$ & $1.407(12)$ \\
\hline$C(31 \mathrm{~A})-\mathrm{C}(32 \mathrm{~A})$ & $1.63(2)$ & $\mathrm{Cl}(2)-\mathrm{O}(201)$ & $1.414(14)$ \\
\hline$C(32 A)-C(33 A)$ & $1.39(2)$ & & \\
\hline$C(33 \mathrm{~A})-\mathrm{C}(34 \mathrm{~A})$ & $1.50(2)$ & $\mathrm{N}(21 \mathrm{~B}) \# 1-\mathrm{Cu}(1)-\mathrm{N}(11 \mathrm{~A})$ & $88.6(4)$ \\
\hline $\mathrm{N}(11 \mathrm{~B})-\mathrm{C}(14 \mathrm{~B})$ & $1.231(13)$ & $\mathrm{N}(21 \mathrm{~B}) \# 1-\mathrm{Cu}(1)-\mathrm{N}(11 \mathrm{~B})$ & $176.4(4)$ \\
\hline $\mathrm{N}(11 \mathrm{~B})-\mathrm{C}(13 \mathrm{~B})$ & $1.358(15)$ & $\mathrm{N}(11 \mathrm{~A})-\mathrm{Cu}(1)-\mathrm{N}(11 \mathrm{~B})$ & $88.1(3)$ \\
\hline $\mathrm{O}(16 \mathrm{~B})-\mathrm{C}(16 \mathrm{~B})$ & $1.194(15)$ & $\mathrm{N}(21 \mathrm{~B}) \# 1-\mathrm{Cu}(1)-\mathrm{N}(21 \mathrm{~A}) \# 2$ & $91.3(3)$ \\
\hline $\mathrm{C}(11 \mathrm{~B})-\mathrm{C}(12 \mathrm{~B})$ & $1.366(18)$ & $\mathrm{N}(11 \mathrm{~A})-\mathrm{Cu}(1)-\mathrm{N}(21 \mathrm{~A}) \# 2$ & $177.7(4)$ \\
\hline $\mathrm{C}(11 \mathrm{~B})-\mathrm{C}(15 \mathrm{~B})$ & $1.411(17)$ & $\mathrm{N}(11 \mathrm{~B})-\mathrm{Cu}(1)-\mathrm{N}(21 \mathrm{~A}) \# 2$ & $92.0(3)$ \\
\hline
\end{tabular}




\begin{tabular}{|c|c|c|c|}
\hline $\mathrm{N}(21 \mathrm{~B}) \# 1-\mathrm{Cu}(1)-\mathrm{O}(2 \mathrm{~W})$ & $93.5(3)$ & $\mathrm{C}(14 \mathrm{~B})-\mathrm{N}(11 \mathrm{~B})-\mathrm{C}(13 \mathrm{~B})$ & $117.7(10)$ \\
\hline $\mathrm{N}(11 \mathrm{~A})-\mathrm{Cu}(1)-\mathrm{O}(2 \mathrm{~W})$ & $89.8(4)$ & $\mathrm{C}(14 \mathrm{~B})-\mathrm{N}(11 \mathrm{~B})-\mathrm{Cu}(1)$ & $123.8(7)$ \\
\hline $\mathrm{N}(11 \mathrm{~B})-\mathrm{Cu}(1)-\mathrm{O}(2 \mathrm{~W})$ & $87.8(3)$ & $\mathrm{C}(13 \mathrm{~B})-\mathrm{N}(11 \mathrm{~B})-\mathrm{Cu}(1)$ & $118.4(8)$ \\
\hline $\mathrm{N}(21 \mathrm{~A}) \# 2-\mathrm{Cu}(1)-\mathrm{O}(2 \mathrm{~W})$ & $92.5(3)$ & $\mathrm{C}(12 \mathrm{~B})-\mathrm{C}(11 \mathrm{~B})-\mathrm{C}(15 \mathrm{~B})$ & $115.9(10)$ \\
\hline $\mathrm{C}(14 \mathrm{~A})-\mathrm{N}(11 \mathrm{~A})-\mathrm{C}(13 \mathrm{~A})$ & $119.4(10)$ & $\mathrm{C}(12 \mathrm{~B})-\mathrm{C}(11 \mathrm{~B})-\mathrm{C}(16 \mathrm{~B})$ & $115.6(11)$ \\
\hline $\mathrm{C}(14 \mathrm{~A})-\mathrm{N}(11 \mathrm{~A})-\mathrm{Cu}(1)$ & 121.0(9) & $\mathrm{C}(15 \mathrm{~B})-\mathrm{C}(11 \mathrm{~B})-\mathrm{C}(16 \mathrm{~B})$ & $128.5(12)$ \\
\hline $\mathrm{C}(13 \mathrm{~A})-\mathrm{N}(11 \mathrm{~A})-\mathrm{Cu}(1)$ & $118.8(7)$ & $\mathrm{C}(13 \mathrm{~B})-\mathrm{C}(12 \mathrm{~B})-\mathrm{C}(11 \mathrm{~B})$ & $120.4(11)$ \\
\hline $\mathrm{C}(15 \mathrm{~A})-\mathrm{C}(11 \mathrm{~A})-\mathrm{C}(12 \mathrm{~A})$ & $116.9(9)$ & $\mathrm{C}(12 \mathrm{~B})-\mathrm{C}(13 \mathrm{~B})-\mathrm{N}(11 \mathrm{~B})$ & $121.2(12)$ \\
\hline$C(15 A)-C(11 A)-C(16 A)$ & $125.7(12)$ & $\mathrm{N}(11 \mathrm{~B})-\mathrm{C}(14 \mathrm{~B})-\mathrm{C}(15 \mathrm{~B})$ & $126.9(11)$ \\
\hline $\mathrm{C}(12 \mathrm{~A})-\mathrm{C}(11 \mathrm{~A})-\mathrm{C}(16 \mathrm{~A})$ & $117.4(11)$ & $\mathrm{C}(14 \mathrm{~B})-\mathrm{C}(15 \mathrm{~B})-\mathrm{C}(11 \mathrm{~B})$ & $117.8(11)$ \\
\hline $\mathrm{C}(13 \mathrm{~A})-\mathrm{C}(12 \mathrm{~A})-\mathrm{C}(11 \mathrm{~A})$ & 119.1(11) & $\mathrm{O}(16 \mathrm{~B})-\mathrm{C}(16 \mathrm{~B})-\mathrm{N}(31 \mathrm{~B})$ & $123.8(11)$ \\
\hline $\mathrm{C}(12 \mathrm{~A})-\mathrm{C}(13 \mathrm{~A})-\mathrm{N}(11 \mathrm{~A})$ & $123.0(10)$ & $\mathrm{O}(16 \mathrm{~B})-\mathrm{C}(16 \mathrm{~B})-\mathrm{C}(11 \mathrm{~B})$ & $121.2(13)$ \\
\hline $\mathrm{N}(11 \mathrm{~A})-\mathrm{C}(14 \mathrm{~A})-\mathrm{C}(15 \mathrm{~A})$ & $117.4(11)$ & $\mathrm{N}(31 \mathrm{~B})-\mathrm{C}(16 \mathrm{~B})-\mathrm{C}(11 \mathrm{~B})$ & $115.0(11)$ \\
\hline $\mathrm{C}(11 \mathrm{~A})-\mathrm{C}(15 \mathrm{~A})-\mathrm{C}(14 \mathrm{~A})$ & $123.7(10)$ & $\mathrm{C}(24 \mathrm{~B})-\mathrm{N}(21 \mathrm{~B})-\mathrm{C}(23 \mathrm{~B})$ & $115.3(9)$ \\
\hline $\mathrm{O}(16 \mathrm{~A})-\mathrm{C}(16 \mathrm{~A})-\mathrm{N}(31 \mathrm{~A})$ & $126.9(12)$ & $\mathrm{C}(24 \mathrm{~B})-\mathrm{N}(21 \mathrm{~B})-\mathrm{Cu}(1) \# 4$ & $124.7(7)$ \\
\hline $\mathrm{O}(16 \mathrm{~A})-\mathrm{C}(16 \mathrm{~A})-\mathrm{C}(11 \mathrm{~A})$ & $116.7(12)$ & $\mathrm{C}(23 \mathrm{~B})-\mathrm{N}(21 \mathrm{~B})-\mathrm{Cu}(1) \# 4$ & $119.9(7)$ \\
\hline $\mathrm{N}(31 \mathrm{~A})-\mathrm{C}(16 \mathrm{~A})-\mathrm{C}(11 \mathrm{~A})$ & $116.5(12)$ & $\mathrm{C}(25 \mathrm{~B})-\mathrm{C}(21 \mathrm{~B})-\mathrm{C}(22 \mathrm{~B})$ & $116.2(10)$ \\
\hline $\mathrm{C}(24 \mathrm{~A})-\mathrm{N}(21 \mathrm{~A})-\mathrm{C}(23 \mathrm{~A})$ & 117.7(9) & $\mathrm{C}(25 \mathrm{~B})-\mathrm{C}(21 \mathrm{~B})-\mathrm{C}(26 \mathrm{~B})$ & 119.1(10) \\
\hline $\mathrm{C}(24 \mathrm{~A})-\mathrm{N}(21 \mathrm{~A})-\mathrm{Cu}(1) \# 3$ & $119.6(7)$ & $\mathrm{C}(22 \mathrm{~B})-\mathrm{C}(21 \mathrm{~B})-\mathrm{C}(26 \mathrm{~B})$ & $124.4(10)$ \\
\hline $\mathrm{C}(23 \mathrm{~A})-\mathrm{N}(21 \mathrm{~A})-\mathrm{Cu}(1) \# 3$ & $122.5(6)$ & $\mathrm{C}(23 \mathrm{~B})-\mathrm{C}(22 \mathrm{~B})-\mathrm{C}(21 \mathrm{~B})$ & $121.7(11)$ \\
\hline $\mathrm{C}(22 \mathrm{~A})-\mathrm{C}(21 \mathrm{~A})-\mathrm{C}(25 \mathrm{~A})$ & 119.3(10) & $\mathrm{C}(22 \mathrm{~B})-\mathrm{C}(23 \mathrm{~B})-\mathrm{N}(21 \mathrm{~B})$ & $122.9(10)$ \\
\hline$C(22 A)-C(21 A)-C(26 A)$ & 119.6(11) & $\mathrm{C}(25 \mathrm{~B})-\mathrm{C}(24 \mathrm{~B})-\mathrm{N}(21 \mathrm{~B})$ & $123.1(10)$ \\
\hline$C(25 A)-C(21 A)-C(26 A)$ & 121.1(12) & $\mathrm{C}(24 \mathrm{~B})-\mathrm{C}(25 \mathrm{~B})-\mathrm{C}(21 \mathrm{~B})$ & $120.7(11)$ \\
\hline $\mathrm{C}(23 \mathrm{~A})-\mathrm{C}(22 \mathrm{~A})-\mathrm{C}(21 \mathrm{~A})$ & $121.0(9)$ & $\mathrm{O}(26 \mathrm{~B})-\mathrm{C}(26 \mathrm{~B})-\mathrm{N}(32 \mathrm{~B})$ & $125.4(10)$ \\
\hline $\mathrm{C}(22 \mathrm{~A})-\mathrm{C}(23 \mathrm{~A})-\mathrm{N}(21 \mathrm{~A})$ & 122.2(9) & $\mathrm{O}(26 \mathrm{~B})-\mathrm{C}(26 \mathrm{~B})-\mathrm{C}(21 \mathrm{~B})$ & $118.8(11)$ \\
\hline $\mathrm{N}(21 \mathrm{~A})-\mathrm{C}(24 \mathrm{~A})-\mathrm{C}(25 \mathrm{~A})$ & $120.8(10)$ & $\mathrm{N}(32 \mathrm{~B})-\mathrm{C}(26 \mathrm{~B})-\mathrm{C}(21 \mathrm{~B})$ & $115.8(10)$ \\
\hline $\mathrm{C}(21 \mathrm{~A})-\mathrm{C}(25 \mathrm{~A})-\mathrm{C}(24 \mathrm{~A})$ & $118.8(11)$ & $\mathrm{C}(16 \mathrm{~B})-\mathrm{N}(31 \mathrm{~B})-\mathrm{C}(31 \mathrm{~B})$ & $121.6(11)$ \\
\hline $\mathrm{O}(26 \mathrm{~A})-\mathrm{C}(26 \mathrm{~A})-\mathrm{N}(32 \mathrm{~A})$ & $124.6(11)$ & $\mathrm{C}(26 \mathrm{~B})-\mathrm{N}(32 \mathrm{~B})-\mathrm{C}(34 \mathrm{~B})$ & $120.7(10)$ \\
\hline $\mathrm{O}(26 \mathrm{~A})-\mathrm{C}(26 \mathrm{~A})-\mathrm{C}(21 \mathrm{~A})$ & 121.2(11) & $\mathrm{N}(31 \mathrm{~B})-\mathrm{C}(31 \mathrm{~B})-\mathrm{C}(32 \mathrm{~B})$ & $110.6(12)$ \\
\hline $\mathrm{N}(32 \mathrm{~A})-\mathrm{C}(26 \mathrm{~A})-\mathrm{C}(21 \mathrm{~A})$ & $114.3(11)$ & $\mathrm{C}(31 \mathrm{~B})-\mathrm{C}(32 \mathrm{~B})-\mathrm{C}(33 \mathrm{~B})$ & $117.3(14)$ \\
\hline$C(16 A)-N(31 A)-C(31 A)$ & 119.1(12) & $\mathrm{C}(34 \mathrm{~B})-\mathrm{C}(33 \mathrm{~B})-\mathrm{C}(32 \mathrm{~B})$ & $117.0(17)$ \\
\hline $\mathrm{C}(26 \mathrm{~A})-\mathrm{N}(32 \mathrm{~A})-\mathrm{C}(34 \mathrm{~A})$ & $122.3(11)$ & $\mathrm{N}(32 \mathrm{~B})-\mathrm{C}(34 \mathrm{~B})-\mathrm{C}(33 \mathrm{~B})$ & $112.3(11)$ \\
\hline $\mathrm{N}(31 \mathrm{~A})-\mathrm{C}(31 \mathrm{~A})-\mathrm{C}(32 \mathrm{~A})$ & $107.4(11)$ & $\mathrm{O}(103)-\mathrm{Cl}(1)-\mathrm{O}(102)$ & $111.7(12)$ \\
\hline $\mathrm{C}(33 \mathrm{~A})-\mathrm{C}(32 \mathrm{~A})-\mathrm{C}(31 \mathrm{~A})$ & $115.4(13)$ & $\mathrm{O}(103)-\mathrm{Cl}(1)-\mathrm{O}(104)$ & $107.0(7)$ \\
\hline $\mathrm{C}(32 \mathrm{~A})-\mathrm{C}(33 \mathrm{~A})-\mathrm{C}(34 \mathrm{~A})$ & $113.9(12)$ & $\mathrm{O}(102)-\mathrm{Cl}(1)-\mathrm{O}(104)$ & $111.8(10)$ \\
\hline $\mathrm{N}(32 \mathrm{~A})-\mathrm{C}(34 \mathrm{~A})-\mathrm{C}(33 \mathrm{~A})$ & $111.2(12)$ & $\mathrm{O}(103)-\mathrm{Cl}(1)-\mathrm{O}(101)$ & $102.3(11)$ \\
\hline
\end{tabular}




$\begin{array}{lllr}\mathrm{O}(102)-\mathrm{Cl}(1)-\mathrm{O}(101) & 107.2(11) & \mathrm{O}(204)-\mathrm{Cl}(2)-\mathrm{O}(202) & 108.4(10) \\ \mathrm{O}(104)-\mathrm{Cl}(1)-\mathrm{O}(101) & 116.5(10) & \mathrm{O}(203)-\mathrm{Cl}(2)-\mathrm{O}(201) & 121.2(15) \\ \mathrm{O}(203)-\mathrm{Cl}(2)-\mathrm{O}(204) & 104(2) & \mathrm{O}(204)-\mathrm{Cl}(2)-\mathrm{O}(201) & 96.7(11) \\ \mathrm{O}(203)-\mathrm{Cl}(2)-\mathrm{O}(202) & 111.6(14) & \mathrm{O}(202)-\mathrm{Cl}(2)-\mathrm{O}(201) & 112.9(10)\end{array}$

Symmetry transformations used to generate equivalent atoms:

\#1 x+1,y,z \#2 x,y+1,z \#3 x,y-1,z \#4 x-1,y,z

Table 4. Anisotropic displacement parameters $\left(\AA^{2} \times 10^{3}\right)$ for 3. The anisotropic

displacement factor exponent takes the form: $-2 \pi^{2}\left[h^{2} a^{* 2} U^{11}+\ldots+2 h k a^{*} b^{*} U^{12}\right]$

\begin{tabular}{|c|c|c|c|c|c|c|}
\hline & $\mathrm{U}^{11}$ & $\mathrm{U}^{22}$ & $\mathrm{U}^{33}$ & $\mathrm{U}^{23}$ & $\mathrm{U}^{13}$ & $\mathrm{U}^{12}$ \\
\hline $\mathrm{Cu}(1)$ & $24(1)$ & $27(1)$ & $54(1)$ & $1(1)$ & $1(1)$ & $13(1)$ \\
\hline $\mathrm{O}(1 \mathrm{~W})$ & $54(5)$ & $54(5)$ & $49(5)$ & $4(4)$ & $-1(3)$ & $23(4)$ \\
\hline $\mathrm{O}(2 \mathrm{~W})$ & $66(5)$ & $74(6)$ & $43(4)$ & 2(4) & $-8(4)$ & $45(5)$ \\
\hline $\mathrm{N}(11 \mathrm{~A})$ & $51(6)$ & $39(5)$ & $67(6)$ & $-17(4)$ & $1(5)$ & $31(5)$ \\
\hline $\mathrm{O}(16 \mathrm{~A})$ & $127(10)$ & $55(6)$ & $91(8)$ & $6(5)$ & $-17(7)$ & $40(6)$ \\
\hline $\mathrm{C}(11 \mathrm{~A})$ & $47(6)$ & $18(5)$ & $85(8)$ & $8(5)$ & $-3(6)$ & $9(5)$ \\
\hline $\mathrm{C}(12 \mathrm{~A})$ & $66(8)$ & $48(7)$ & $86(9)$ & $19(6)$ & $38(7)$ & $40(6)$ \\
\hline $\mathrm{C}(13 \mathrm{~A})$ & $52(7)$ & $28(5)$ & $62(7)$ & $-3(5)$ & $11(5)$ & $17(5)$ \\
\hline $\mathrm{C}(14 \mathrm{~A})$ & $61(7)$ & $55(7)$ & $60(7)$ & $-5(5)$ & $-10(6)$ & $51(6)$ \\
\hline $\mathrm{C}(15 \mathrm{~A})$ & $51(6)$ & $33(6)$ & $88(9)$ & $-28(6)$ & $1(6)$ & $32(5)$ \\
\hline$C(16 A)$ & 101(11) & $36(6)$ & $64(8)$ & $14(6)$ & 1(7) & $30(7)$ \\
\hline $\mathrm{N}(21 \mathrm{~A})$ & $32(4)$ & $28(4)$ & $51(5)$ & $21(4)$ & $-1(4)$ & $4(4)$ \\
\hline $\mathrm{O}(26 \mathrm{~A})$ & $70(6)$ & $40(4)$ & $70(5)$ & $-11(4)$ & $-10(5)$ & $28(4)$ \\
\hline $\mathrm{C}(21 \mathrm{~A})$ & 74(9) & $27(5)$ & $73(8)$ & $-15(5)$ & $-3(7)$ & $26(6)$ \\
\hline $\mathrm{C}(22 \mathrm{~A})$ & $48(6)$ & $26(5)$ & $50(6)$ & $-10(4)$ & $-21(5)$ & $14(5)$ \\
\hline $\mathrm{C}(23 \mathrm{~A})$ & $55(6)$ & $26(5)$ & $43(5)$ & $-5(4)$ & $-37(5)$ & $13(5)$ \\
\hline $\mathrm{C}(24 \mathrm{~A})$ & $46(6)$ & $29(5)$ & $49(6)$ & 1(4) & $-7(5)$ & $17(5)$ \\
\hline $\mathrm{C}(25 \mathrm{~A})$ & $52(7)$ & $47(6)$ & $46(6)$ & $-10(5)$ & $-12(5)$ & $28(5)$ \\
\hline$C(26 A)$ & $58(7)$ & $35(6)$ & $64(7)$ & $7(5)$ & $8(6)$ & $28(6)$ \\
\hline $\mathrm{N}(31 \mathrm{~A})$ & 94(9) & $49(6)$ & $80(7)$ & $-21(6)$ & $-30(7)$ & $45(6)$ \\
\hline $\mathrm{N}(32 \mathrm{~A})$ & 102(9) & $52(6)$ & $66(6)$ & $1(5)$ & $-9(6)$ & $50(6)$ \\
\hline $\mathrm{C}(31 \mathrm{~A})$ & 77(9) & $26(6)$ & $120(12)$ & $-8(7)$ & $17(8)$ & $30(6)$ \\
\hline $\mathrm{C}(32 \mathrm{~A})$ & $80(9)$ & $60(8)$ & $98(11)$ & $10(7)$ & $-14(8)$ & $55(8)$ \\
\hline $\mathrm{C}(33 \mathrm{~A})$ & $82(9)$ & $39(6)$ & $54(7)$ & $-13(5)$ & $-14(7)$ & $19(6)$ \\
\hline
\end{tabular}




\begin{tabular}{|c|c|c|c|c|c|c|}
\hline$C(34 \mathrm{~A})$ & $83(9)$ & $60(8)$ & $67(8)$ & $-9(6)$ & 2(7) & $46(7)$ \\
\hline $\mathrm{N}(11 \mathrm{~B})$ & $33(4)$ & $22(4)$ & $72(6)$ & $-3(4)$ & $8(4)$ & $17(4)$ \\
\hline $\mathrm{O}(16 \mathrm{~B})$ & $37(5)$ & 113(9) & $97(7)$ & $7(6)$ & $6(5)$ & $36(5)$ \\
\hline$C(11 B)$ & $49(7)$ & $67(8)$ & $69(7)$ & $0(6)$ & $-5(6)$ & $46(6)$ \\
\hline$C(12 B)$ & $32(5)$ & $38(6)$ & $85(8)$ & $-12(6)$ & $25(5)$ & $16(5)$ \\
\hline$C(13 B)$ & $44(7)$ & $49(7)$ & $85(9)$ & $1(6)$ & $6(6)$ & $25(6)$ \\
\hline$C(14 B)$ & $30(5)$ & $42(6)$ & $64(7)$ & $-21(5)$ & $-17(5)$ & $23(5)$ \\
\hline$C(15 B)$ & $39(6)$ & $72(9)$ & $103(11)$ & $-57(8)$ & $-26(7)$ & $27(6)$ \\
\hline$C(16 B)$ & $48(7)$ & $49(7)$ & $75(8)$ & $14(6)$ & $23(6)$ & $29(6)$ \\
\hline $\mathrm{N}(21 \mathrm{~B})$ & $47(5)$ & $46(5)$ & $36(4)$ & $-2(4)$ & $7(4)$ & $38(4)$ \\
\hline $\mathrm{O}(26 \mathrm{~B})$ & $24(4)$ & $63(6)$ & $101(7)$ & $-11(5)$ & $6(4)$ & $9(4)$ \\
\hline$C(21 B)$ & $34(5)$ & $52(6)$ & $37(5)$ & $-5(5)$ & $2(4)$ & $23(5)$ \\
\hline$C(22 B)$ & $50(7)$ & $71(8)$ & $40(6)$ & $4(5)$ & $7(5)$ & $36(6)$ \\
\hline$C(23 B)$ & $43(6)$ & $31(5)$ & $38(5)$ & $7(4)$ & $9(4)$ & $11(4)$ \\
\hline$C(24 B)$ & $47(7)$ & $45(6)$ & $55(7)$ & $23(5)$ & $2(5)$ & $13(5)$ \\
\hline$C(25 B)$ & $72(9)$ & $40(6)$ & $50(6)$ & $13(5)$ & $1(6)$ & $21(6)$ \\
\hline$C(26 B)$ & $19(5)$ & $69(8)$ & $51(6)$ & $-3(5)$ & $7(4)$ & $19(5)$ \\
\hline $\mathrm{N}(31 \mathrm{~B})$ & $49(6)$ & $117(10)$ & $52(6)$ & $-10(6)$ & $-3(5)$ & $42(7)$ \\
\hline $\mathrm{N}(32 \mathrm{~B})$ & $27(4)$ & $60(6)$ & $91(7)$ & $-7(5)$ & $13(5)$ & $26(4)$ \\
\hline$C(31 B)$ & $20(5)$ & $88(10)$ & $80(9)$ & $-4(7)$ & $-10(5)$ & $20(6)$ \\
\hline$C(32 B)$ & $68(9)$ & $100(12)$ & $88(10)$ & 19(9) & $-1(8)$ & $47(9)$ \\
\hline$C(33 B)$ & $85(11)$ & $147(17)$ & $108(12)$ & $-58(12)$ & $-40(9)$ & $97(12)$ \\
\hline$C(34 B)$ & $44(7)$ & $80(9)$ & $63(8)$ & 13(7) & $8(6)$ & $28(7)$ \\
\hline $\mathrm{Cl}(1)$ & $64(2)$ & $46(2)$ & $81(2)$ & $-4(2)$ & $16(2)$ & $25(2)$ \\
\hline $\mathrm{O}(101)$ & $68(8)$ & $116(12)$ & $280(20)$ & $28(13)$ & $-60(11)$ & $39(8)$ \\
\hline $\mathrm{O}(102)$ & $220(20)$ & $51(7)$ & $179(15)$ & $-45(8)$ & $-33(14)$ & $70(10)$ \\
\hline $\mathrm{O}(103)$ & $149(11)$ & $188(14)$ & $75(7)$ & $-3(8)$ & $26(7)$ & $146(12)$ \\
\hline $\mathrm{O}(104)$ & $152(11)$ & $78(7)$ & $92(7)$ & $20(6)$ & $56(8)$ & $75(8)$ \\
\hline $\mathrm{Cl}(2)$ & $72(2)$ & $69(2)$ & $57(2)$ & $-5(2)$ & $-16(2)$ & $39(2)$ \\
\hline $\mathrm{O}(201)$ & 153(14) & 189(16) & $80(8)$ & $56(9)$ & $7(8)$ & $90(13)$ \\
\hline $\mathrm{O}(202)$ & $96(9)$ & 253(19) & $48(6)$ & $-15(8)$ & $-18(6)$ & $98(11)$ \\
\hline $\mathrm{O}(203)$ & $450(50)$ & $55(9)$ & $160(16)$ & $44(10)$ & $-50(20)$ & $13(16)$ \\
\hline $\mathrm{O}(204)$ & $110(12)$ & $280(30)$ & $112(11)$ & $-11(14)$ & $-21(10)$ & $89(15)$ \\
\hline $\mathrm{O}(3 \mathrm{~W})$ & $190(20)$ & $250(30)$ & $190(20)$ & $0(20)$ & $-21(18)$ & $110(20)$ \\
\hline $\mathrm{O}(4 \mathrm{~W})$ & $490(70)$ & $180(30)$ & $350(50)$ & $30(30)$ & $-100(50)$ & $100(40)$ \\
\hline
\end{tabular}


Table 5. Hydrogen coordinates ( $\left.\mathrm{x} 10^{4}\right)$ and isotropic displacement parameters $\left(\AA^{2} \mathrm{x} 10^{3}\right)$ for 3 .

\begin{tabular}{|c|c|c|c|c|}
\hline & $\mathrm{x}$ & $\mathrm{y}$ & $\mathrm{z}$ & $\mathrm{U}(\mathrm{eq})$ \\
\hline $\mathrm{H}(12 \mathrm{~A})$ & 5974 & 17808 & 5159 & 74 \\
\hline $\mathrm{H}(13 \mathrm{~A})$ & 6262 & 19165 & 4837 & 58 \\
\hline $\mathrm{H}(14 \mathrm{~A})$ & 7671 & 19358 & 2424 & 59 \\
\hline $\mathrm{H}(15 \mathrm{~A})$ & 7287 & 17902 & 2692 & 63 \\
\hline $\mathrm{H}(22 \mathrm{~A})$ & 9046 & 13744 & 4522 & 52 \\
\hline $\mathrm{H}(23 \mathrm{~A})$ & 8617 & 12342 & 4582 & 53 \\
\hline $\mathrm{H}(24 \mathrm{~A})$ & 6877 & 11845 & 2489 & 50 \\
\hline $\mathrm{H}(25 \mathrm{~A})$ & 7342 & 13305 & 2377 & 56 \\
\hline $\mathrm{H}(31 \mathrm{~A})$ & 6354 & 16437 & 2939 & 84 \\
\hline $\mathrm{H}(32 \mathrm{~A})$ & 8132 & 14585 & 2107 & 81 \\
\hline $\mathrm{H}(31 \mathrm{~B})$ & 5957 & 15012 & 3357 & 87 \\
\hline $\mathrm{H}(31 \mathrm{C})$ & 6175 & 15298 & 4493 & 87 \\
\hline $\mathrm{H}(32 \mathrm{~B})$ & 7715 & 16060 & 4088 & 84 \\
\hline $\mathrm{H}(32 \mathrm{C})$ & 7315 & 15078 & 3860 & 84 \\
\hline $\mathrm{H}(33 \mathrm{~A})$ & 7732 & 16354 & 2488 & 76 \\
\hline $\mathrm{H}(33 \mathrm{~B})$ & 7213 & 15375 & 2211 & 76 \\
\hline $\mathrm{H}(34 \mathrm{~A})$ & 8701 & 16015 & 1860 & 79 \\
\hline $\mathrm{H}(34 \mathrm{~B})$ & 8884 & 16157 & 3022 & 79 \\
\hline $\mathrm{H}(12 \mathrm{~B})$ & 4816 & 20765 & 4741 & 63 \\
\hline $\mathrm{H}(13 \mathrm{~B})$ & 6232 & 21144 & 4616 & 70 \\
\hline $\mathrm{H}(14 \mathrm{~B})$ & 5735 & 19519 & 2504 & 52 \\
\hline $\mathrm{H}(15 \mathrm{~B})$ & 4311 & 19002 & 2600 & 86 \\
\hline $\mathrm{H}(22 \mathrm{~B})$ & -234 & 20281 & 4611 & 62 \\
\hline $\mathrm{H}(23 \mathrm{~B})$ & -1551 & 20129 & 4639 & 48 \\
\hline $\mathrm{H}(24 \mathrm{~B})$ & -906 & 21811 & 2425 & 65 \\
\hline $\mathrm{H}(25 \mathrm{~B})$ & 458 & 22040 & 2442 & 68 \\
\hline $\mathrm{H}(31 \mathrm{D})$ & 3064 & 18972 & 2667 & 87 \\
\hline $\mathrm{H}(32 \mathrm{D})$ & 855 & 20214 & 3979 & 69 \\
\hline $\mathrm{H}(31 \mathrm{E})$ & 1858 & 19004 & 3909 & 79 \\
\hline $\mathrm{H}(31 \mathrm{~F})$ & 1630 & 18407 & 2957 & 79 \\
\hline $\mathrm{H}(32 \mathrm{E})$ & 1330 & 19445 & 2601 & 100 \\
\hline
\end{tabular}




$\begin{array}{lrrrr}\mathrm{H}(32 \mathrm{~F}) & 2104 & 19581 & 1931 & 100 \\ \mathrm{H}(33 \mathrm{C}) & 3048 & 20711 & 3044 & 114 \\ \mathrm{H}(33 \mathrm{D}) & 2431 & 20918 & 2429 & 114 \\ \mathrm{H}(34 \mathrm{C}) & 2163 & 20417 & 4454 & 76 \\ \mathrm{H}(34 \mathrm{D}) & 2584 & 21377 & 4095 & 76 \\ \end{array}$

Table 1. Crystal data and structure refinement for 4 .

Identification code

Empirical formula

Formula weight

Temperature

Wavelength

Crystal system

Space group

Unit cell dimensions

Volume

$\mathrm{Z}$

Density (calculated)

Absorption coefficient

$\mathrm{F}(000)$

Crystal size

Theta range for data collection

Index ranges

Reflections collected

Independent reflections

Completeness to theta $=24.98^{\circ}$

Absorption correction

Refinement method

Data / restraints / parameters

Goodness-of-fit on $\mathrm{F}^{2}$

Final $\mathrm{R}$ indices [I>2sigma(I)]

$\mathrm{R}$ indices (all data)

Absolute structure parameter

Largest diff. peak and hole

\section{4}

C32 H40 Cu F12 N8 O6 P2

986.20

293(2) K

$0.71073 \AA$

Tetragonal

I-4

$\mathrm{a}=12.4930(18) \AA \quad \alpha=90^{\circ}$.

$\mathrm{b}=12.4930(18) \AA \quad \beta=90^{\circ}$.

$\mathrm{c}=31.885(6) \AA \quad \gamma=90^{\circ}$.

4976.5(14) $\AA^{3}$

4

$1.316 \mathrm{Mg} / \mathrm{m}^{3}$

$0.592 \mathrm{~mm}^{-1}$

2012

$0.15 \times 0.15 \times 0.15 \mathrm{~mm}^{3}$

1.28 to $24.98^{\circ}$.

$-14<=\mathrm{h}<=14,-14<=\mathrm{k}<=14,-37<=\mathrm{l}<=37$

4823

$4380[\mathrm{R}(\mathrm{int})=0.0232]$

$100.0 \%$

None

Full-matrix least-squares on $\mathrm{F}^{2}$

4380 / 0 / 249

1.114

$\mathrm{R} 1=0.0492, \mathrm{wR} 2=0.1085$

$\mathrm{R} 1=0.0664, \mathrm{wR} 2=0.1316$

$-0.07(2)$

0.244 and -0.330 e. $\AA^{-3}$ 
Table 2. Atomic coordinates ( $\times 10^{4}$ ) and equivalent isotropic displacement parameters $\left(\AA^{2} \times 10^{3}\right)$ for 4. $U(e q)$ is defined as one third of the trace of the orthogonalized $U^{\mathrm{ij}}$ tensor.

\begin{tabular}{|c|c|c|c|c|}
\hline & $\mathrm{x}$ & $\mathrm{y}$ & $\mathrm{z}$ & $\mathrm{U}(\mathrm{eq})$ \\
\hline $\mathrm{Cu}(1)$ & 0 & 10000 & $12213(1)$ & $48(1)$ \\
\hline $\mathrm{P}(1)$ & 10000 & 5000 & 7500 & $61(1)$ \\
\hline $\mathrm{F}(11)$ & 10000 & 5000 & 7990(2) & $119(2)$ \\
\hline $\mathrm{F}(12)$ & $9219(3)$ & 3993(3) & 7493(1) & $98(1)$ \\
\hline $\mathrm{P}(2)$ & 10000 & 0 & 10000 & $59(1)$ \\
\hline $\mathrm{F}(21)$ & $9825(4)$ & $1203(4)$ & $10034(4)$ & $188(3)$ \\
\hline $\mathrm{F}(22)$ & $10513(11)$ & $45(19)$ & $10429(3)$ & $183(8)$ \\
\hline $\mathrm{N}(11)$ & $1003(3)$ & $9368(3)$ & $11762(1)$ & $50(1)$ \\
\hline $\mathrm{O}(10)$ & $-1070(3)$ & $8357(3)$ & $12162(1)$ & $64(1)$ \\
\hline $\mathrm{O}(16)$ & $4048(3)$ & $8107(4)$ & $10876(1)$ & $77(1)$ \\
\hline $\mathrm{C}(11)$ & $617(4)$ & $8855(5)$ & $11435(1)$ & $56(1)$ \\
\hline$C(12)$ & $1268(4)$ & $8499(5)$ & $11121(1)$ & $57(1)$ \\
\hline $\mathrm{C}(13)$ & 2354(4) & $8653(4)$ & 11131(1) & $45(1)$ \\
\hline$C(14)$ & $2755(4)$ & 9150(4) & 11489(1) & $48(1)$ \\
\hline$C(15)$ & 2041(4) & $9475(4)$ & $11796(1)$ & $48(1)$ \\
\hline$C(16)$ & $3133(4)$ & $8310(4)$ & 10794(1) & $51(1)$ \\
\hline $\mathrm{N}(21)$ & $4043(3)$ & $5596(3)$ & $7673(1)$ & $43(1)$ \\
\hline $\mathrm{O}(26)$ & $1850(3)$ & 7902(3) & $8618(1)$ & $60(1)$ \\
\hline $\mathrm{C}(21)$ & $4050(4)$ & 6624(4) & $7765(2)$ & $69(2)$ \\
\hline $\mathrm{C}(22)$ & $3443(5)$ & 7068(4) & $8083(2)$ & $62(2)$ \\
\hline $\mathrm{C}(23)$ & $2786(4)$ & 6442(4) & $8316(1)$ & $45(1)$ \\
\hline $\mathrm{C}(24)$ & $2778(4)$ & $5365(4)$ & $8223(2)$ & $59(1)$ \\
\hline$C(25)$ & $3414(5)$ & 4986(4) & $7905(2)$ & $62(1)$ \\
\hline$C(26)$ & 2106(4) & 6932(4) & $8649(1)$ & $48(1)$ \\
\hline $\mathrm{N}(31)$ & 2720(4) & 8292(4) & $10417(1)$ & $64(1)$ \\
\hline $\mathrm{N}(32)$ & 1839(3) & $6325(3)$ & 8973(1) & $50(1)$ \\
\hline $\mathrm{C}(31)$ & $3370(5)$ & $7970(6)$ & $10044(2)$ & $83(2)$ \\
\hline $\mathrm{C}(32)$ & $2678(5)$ & 7766(6) & $9677(2)$ & $66(2)$ \\
\hline $\mathrm{C}(33)$ & 1892(5) & $6877(5)$ & $9712(2)$ & $63(2)$ \\
\hline $\mathrm{C}(34)$ & $1208(5)$ & $6712(5)$ & $9326(2)$ & $61(1)$ \\
\hline
\end{tabular}


Table 3. Bond lengths $[\AA ̊]$ and angles $\left[{ }^{\circ}\right]$ for 4 .

\begin{tabular}{|c|c|c|c|}
\hline $\mathrm{Cu}(1)-\mathrm{N}(21) \# 1$ & $2.033(4)$ & $\mathrm{C}(21)-\mathrm{C}(22)$ & $1.382(7)$ \\
\hline $\mathrm{Cu}(1)-\mathrm{N}(21) \# 2$ & $2.033(4)$ & $\mathrm{C}(22)-\mathrm{C}(23)$ & $1.356(7)$ \\
\hline $\mathrm{Cu}(1)-\mathrm{N}(11) \# 3$ & $2.064(4)$ & $\mathrm{C}(23)-\mathrm{C}(24)$ & $1.377(7)$ \\
\hline $\mathrm{Cu}(1)-\mathrm{N}(11)$ & $2.064(4)$ & $C(23)-C(26)$ & $1.493(6)$ \\
\hline $\mathrm{P}(1)-\mathrm{F}(11)$ & $1.563(5)$ & $C(24)-C(25)$ & $1.372(7)$ \\
\hline $\mathrm{P}(1)-\mathrm{F}(11) \# 4$ & $1.563(5)$ & $\mathrm{C}(26)-\mathrm{N}(32)$ & $1.322(6)$ \\
\hline $\mathrm{P}(1)-\mathrm{F}(12) \# 5$ & $1.592(4)$ & $\mathrm{N}(31)-\mathrm{C}(31)$ & $1.497(7)$ \\
\hline$P(1)-F(12)$ & $1.592(4)$ & $\mathrm{N}(32)-\mathrm{C}(34)$ & $1.459(6)$ \\
\hline $\mathrm{P}(1)-\mathrm{F}(12) \# 6$ & $1.592(4)$ & $\mathrm{C}(31)-\mathrm{C}(32)$ & $1.477(9)$ \\
\hline $\mathrm{P}(1)-\mathrm{F}(12) \# 4$ & $1.592(4)$ & $\mathrm{C}(32)-\mathrm{C}(33)$ & $1.487(9)$ \\
\hline$P(2)-F(22)$ & $1.511(9)$ & $\mathrm{C}(33)-\mathrm{C}(34)$ & $1.511(8)$ \\
\hline $\mathrm{P}(2)-\mathrm{F}(22) \# 7$ & $1.511(9)$ & & \\
\hline $\mathrm{P}(2)-\mathrm{F}(22) \# 8$ & $1.511(9)$ & $\mathrm{N}(21) \# 1-\mathrm{Cu}(1)-\mathrm{N}(21) \# 2$ & $87.7(2)$ \\
\hline$P(2)-F(22) \# 9$ & $1.511(9)$ & $\mathrm{N}(21) \# 1-\mathrm{Cu}(1)-\mathrm{N}(11) \# 3$ & $177.97(16)$ \\
\hline$P(2)-F(21)$ & $1.522(5)$ & $\mathrm{N}(21) \# 2-\mathrm{Cu}(1)-\mathrm{N}(11) \# 3$ & $90.31(13)$ \\
\hline $\mathrm{P}(2)-\mathrm{F}(21) \# 8$ & $1.522(5)$ & $\mathrm{N}(21) \# 1-\mathrm{Cu}(1)-\mathrm{N}(11)$ & $90.31(13)$ \\
\hline $\mathrm{P}(2)-\mathrm{F}(21) \# 9$ & $1.522(5)$ & $\mathrm{N}(21) \# 2-\mathrm{Cu}(1)-\mathrm{N}(11)$ & $177.97(16)$ \\
\hline $\mathrm{P}(2)-\mathrm{F}(21) \# 7$ & $1.522(5)$ & $\mathrm{N}(11) \# 3-\mathrm{Cu}(1)-\mathrm{N}(11)$ & $91.7(2)$ \\
\hline $\mathrm{F}(21)-\mathrm{F}(22) \# 8$ & $1.715(14)$ & $\mathrm{F}(11)-\mathrm{P}(1)-\mathrm{F}(11) \# 4$ & $180.000(2)$ \\
\hline $\mathrm{F}(22)-\mathrm{F}(22) \# 7$ & $1.29(3)$ & $\mathrm{F}(11)-\mathrm{P}(1)-\mathrm{F}(12) \# 5$ & $90.81(16)$ \\
\hline $\mathrm{F}(22)-\mathrm{F}(21) \# 9$ & $1.715(14)$ & $\mathrm{F}(11) \# 4-\mathrm{P}(1)-\mathrm{F}(12) \# 5$ & $89.19(16)$ \\
\hline $\mathrm{N}(11)-\mathrm{C}(15)$ & $1.308(6)$ & $\mathrm{F}(11)-\mathrm{P}(1)-\mathrm{F}(12)$ & $90.81(16)$ \\
\hline $\mathrm{N}(11)-\mathrm{C}(11)$ & $1.317(6)$ & $\mathrm{F}(11) \# 4-\mathrm{P}(1)-\mathrm{F}(12)$ & $89.19(16)$ \\
\hline $\mathrm{O}(16)-\mathrm{C}(16)$ & $1.200(6)$ & $\mathrm{F}(12) \# 5-\mathrm{P}(1)-\mathrm{F}(12)$ & $178.4(3)$ \\
\hline$C(11)-C(12)$ & $1.363(6)$ & $\mathrm{F}(11)-\mathrm{P}(1)-\mathrm{F}(12) \# 6$ & $89.19(16)$ \\
\hline$C(12)-C(13)$ & $1.371(7)$ & $\mathrm{F}(11) \# 4-\mathrm{P}(1)-\mathrm{F}(12) \# 6$ & $90.81(16)$ \\
\hline$C(13)-C(14)$ & $1.394(6)$ & $\mathrm{F}(12) \# 5-\mathrm{P}(1)-\mathrm{F}(12) \# 6$ & $90.012(6)$ \\
\hline$C(13)-C(16)$ & $1.512(6)$ & $\mathrm{F}(12)-\mathrm{P}(1)-\mathrm{F}(12) \# 6$ & $90.011(5)$ \\
\hline$C(14)-C(15)$ & $1.385(6)$ & $\mathrm{F}(11)-\mathrm{P}(1)-\mathrm{F}(12) \# 4$ & $89.19(16)$ \\
\hline $\mathrm{C}(16)-\mathrm{N}(31)$ & $1.308(6)$ & $\mathrm{F}(11) \# 4-\mathrm{P}(1)-\mathrm{F}(12) \# 4$ & $90.81(16)$ \\
\hline $\mathrm{N}(21)-\mathrm{C}(21)$ & $1.318(7)$ & $\mathrm{F}(12) \# 5-\mathrm{P}(1)-\mathrm{F}(12) \# 4$ & $90.011(5)$ \\
\hline $\mathrm{N}(21)-\mathrm{C}(25)$ & $1.321(6)$ & $\mathrm{F}(12)-\mathrm{P}(1)-\mathrm{F}(12) \# 4$ & $90.011(6)$ \\
\hline $\mathrm{N}(21)-\mathrm{Cu}(1) \# 10$ & $2.033(4)$ & $\mathrm{F}(12) \# 6-\mathrm{P}(1)-\mathrm{F}(12) \# 4$ & $178.4(3)$ \\
\hline $\mathrm{O}(26)-\mathrm{C}(26)$ & $1.258(6)$ & $\mathrm{F}(22)-\mathrm{P}(2)-\mathrm{F}(22) \# 7$ & $50.4(11)$ \\
\hline
\end{tabular}




\begin{tabular}{|c|c|c|c|}
\hline $\mathrm{F}(22)-\mathrm{P}(2)-\mathrm{F}(22) \# 8$ & $144.9(7)$ & $\mathrm{C}(12)-\mathrm{C}(13)-\mathrm{C}(14)$ & $115.9(4)$ \\
\hline $\mathrm{F}(22) \# 7-\mathrm{P}(2)-\mathrm{F}(22) \# 8$ & $144.9(7)$ & $\mathrm{C}(12)-\mathrm{C}(13)-\mathrm{C}(16)$ & $125.6(4)$ \\
\hline $\mathrm{F}(22)-\mathrm{P}(2)-\mathrm{F}(22) \# 9$ & $144.9(7)$ & $\mathrm{C}(14)-\mathrm{C}(13)-\mathrm{C}(16)$ & $118.5(4)$ \\
\hline $\mathrm{F}(22) \# 7-\mathrm{P}(2)-\mathrm{F}(22) \# 9$ & $144.9(7)$ & $\mathrm{C}(15)-\mathrm{C}(14)-\mathrm{C}(13)$ & $118.5(4)$ \\
\hline $\mathrm{F}(22) \# 8-\mathrm{P}(2)-\mathrm{F}(22) \# 9$ & $50.4(11)$ & $\mathrm{N}(11)-\mathrm{C}(15)-\mathrm{C}(14)$ & $123.4(4)$ \\
\hline $\mathrm{F}(22)-\mathrm{P}(2)-\mathrm{F}(21)$ & $87.7(10)$ & $\mathrm{O}(16)-\mathrm{C}(16)-\mathrm{N}(31)$ & $125.0(5)$ \\
\hline $\mathrm{F}(22) \# 7-\mathrm{P}(2)-\mathrm{F}(21)$ & $85.0(9)$ & $\mathrm{O}(16)-\mathrm{C}(16)-\mathrm{C}(13)$ & $121.2(4)$ \\
\hline $\mathrm{F}(22) \# 8-\mathrm{P}(2)-\mathrm{F}(21)$ & $68.9(6)$ & $\mathrm{N}(31)-\mathrm{C}(16)-\mathrm{C}(13)$ & $113.8(4)$ \\
\hline $\mathrm{F}(22) \# 9-\mathrm{P}(2)-\mathrm{F}(21)$ & 119.2(8) & $\mathrm{C}(21)-\mathrm{N}(21)-\mathrm{C}(25)$ & $116.2(4)$ \\
\hline $\mathrm{F}(22)-\mathrm{P}(2)-\mathrm{F}(21) \# 8$ & 119.2(8) & $\mathrm{C}(21)-\mathrm{N}(21)-\mathrm{Cu}(1) \# 10$ & $120.9(3)$ \\
\hline $\mathrm{F}(22) \# 7-\mathrm{P}(2)-\mathrm{F}(21) \# 8$ & $68.9(6)$ & $\mathrm{C}(25)-\mathrm{N}(21)-\mathrm{Cu}(1) \# 10$ & $122.9(3)$ \\
\hline $\mathrm{F}(22) \# 8-\mathrm{P}(2)-\mathrm{F}(21) \# 8$ & $87.7(10)$ & $\mathrm{N}(21)-\mathrm{C}(21)-\mathrm{C}(22)$ & $123.4(5)$ \\
\hline $\mathrm{F}(22) \# 9-\mathrm{P}(2)-\mathrm{F}(21) \# 8$ & $85.0(9)$ & $\mathrm{C}(23)-\mathrm{C}(22)-\mathrm{C}(21)$ & $120.2(5)$ \\
\hline $\mathrm{F}(21)-\mathrm{P}(2)-\mathrm{F}(21) \# 8$ & $90.28(7)$ & $\mathrm{C}(22)-\mathrm{C}(23)-\mathrm{C}(24)$ & $116.8(4)$ \\
\hline $\mathrm{F}(22)-\mathrm{P}(2)-\mathrm{F}(21) \# 9$ & $68.9(6)$ & $\mathrm{C}(22)-\mathrm{C}(23)-\mathrm{C}(26)$ & $119.9(4)$ \\
\hline $\mathrm{F}(22) \# 7-\mathrm{P}(2)-\mathrm{F}(21) \# 9$ & $119.2(8)$ & $C(24)-C(23)-C(26)$ & $123.3(4)$ \\
\hline $\mathrm{F}(22) \# 8-\mathrm{P}(2)-\mathrm{F}(21) \# 9$ & $85.0(9)$ & $C(25)-C(24)-C(23)$ & $119.4(5)$ \\
\hline $\mathrm{F}(22) \# 9-\mathrm{P}(2)-\mathrm{F}(21) \# 9$ & $87.7(10)$ & $\mathrm{N}(21)-\mathrm{C}(25)-\mathrm{C}(24)$ & $124.0(5)$ \\
\hline $\mathrm{F}(21)-\mathrm{P}(2)-\mathrm{F}(21) \# 9$ & $90.28(7)$ & $\mathrm{O}(26)-\mathrm{C}(26)-\mathrm{N}(32)$ & $123.5(4)$ \\
\hline $\mathrm{F}(21) \# 8-\mathrm{P}(2)-\mathrm{F}(21) \# 9$ & $172.0(9)$ & $\mathrm{O}(26)-\mathrm{C}(26)-\mathrm{C}(23)$ & $118.9(4)$ \\
\hline $\mathrm{F}(22)-\mathrm{P}(2)-\mathrm{F}(21) \# 7$ & $85.0(9)$ & $\mathrm{N}(32)-\mathrm{C}(26)-\mathrm{C}(23)$ & $117.6(4)$ \\
\hline $\mathrm{F}(22) \# 7-\mathrm{P}(2)-\mathrm{F}(21) \# 7$ & $87.7(10)$ & $\mathrm{C}(16)-\mathrm{N}(31)-\mathrm{C}(31)$ & $121.4(5)$ \\
\hline $\mathrm{F}(22) \# 8-\mathrm{P}(2)-\mathrm{F}(21) \# 7$ & 119.2(8) & $\mathrm{C}(26)-\mathrm{N}(32)-\mathrm{C}(34)$ & $123.3(4)$ \\
\hline $\mathrm{F}(22) \# 9-\mathrm{P}(2)-\mathrm{F}(21) \# 7$ & $68.9(6)$ & $\mathrm{C}(32)-\mathrm{C}(31)-\mathrm{N}(31)$ & $111.0(4)$ \\
\hline $\mathrm{F}(21)-\mathrm{P}(2)-\mathrm{F}(21) \# 7$ & $172.0(9)$ & $\mathrm{C}(31)-\mathrm{C}(32)-\mathrm{C}(33)$ & $117.2(5)$ \\
\hline $\mathrm{F}(21) \# 8-\mathrm{P}(2)-\mathrm{F}(21) \# 7$ & $90.28(7)$ & $\mathrm{C}(32)-\mathrm{C}(33)-\mathrm{C}(34)$ & $114.6(5)$ \\
\hline $\mathrm{F}(21) \# 9-\mathrm{P}(2)-\mathrm{F}(21) \# 7$ & $90.28(7)$ & $\mathrm{N}(32)-\mathrm{C}(34)-\mathrm{C}(33)$ & $111.6(4)$ \\
\hline $\mathrm{P}(2)-\mathrm{F}(21)-\mathrm{F}(22) \# 8$ & $55.3(5)$ & & \\
\hline $\mathrm{F}(22) \# 7-\mathrm{F}(22)-\mathrm{P}(2)$ & $64.8(5)$ & & \\
\hline $\mathrm{F}(22) \# 7-\mathrm{F}(22)-\mathrm{F}(21) \# 9$ & $120.6(5)$ & & \\
\hline $\mathrm{P}(2)-\mathrm{F}(22)-\mathrm{F}(21) \# 9$ & $55.9(4)$ & & \\
\hline $\mathrm{C}(15)-\mathrm{N}(11)-\mathrm{C}(11)$ & $118.6(4)$ & & \\
\hline $\mathrm{C}(15)-\mathrm{N}(11)-\mathrm{Cu}(1)$ & $120.3(3)$ & & \\
\hline $\mathrm{C}(11)-\mathrm{N}(11)-\mathrm{Cu}(1)$ & $121.0(3)$ & & \\
\hline $\mathrm{N}(11)-\mathrm{C}(11)-\mathrm{C}(12)$ & $121.4(5)$ & & \\
\hline $\mathrm{C}(11)-\mathrm{C}(12)-\mathrm{C}(13)$ & $121.9(4)$ & & \\
\hline
\end{tabular}


Symmetry transformations used to generate equivalent atoms:

\#1 -x+1/2,-y+3/2,z+1/2 \#2 x-1/2,y+1/2,z+1/2; \#3 -x,-y+2,z \#4 y+1/2,-x+3/2,-z+3/2 \#5 -x+2,-y+1,z \#6 -y+3/2,x-1/2,-z+3/2 \#7 -x+2,-y,z \#8 -y+1,x-1,-z+2 \#9 y+1,-x+1,-z+2 \#10 x+1/2,y-1/2,z-1/2

Table 4. Anisotropic displacement parameters $\left(\AA^{2} \times 10^{3}\right)$ for 4 . The anisotropic displacement factor exponent takes the form: $-2 \pi^{2}\left[h^{2} a^{* 2} U^{11}+\ldots+2 h k a^{*} b^{*} U^{12}\right]$

\begin{tabular}{|c|c|c|c|c|c|c|}
\hline & $\mathrm{U}^{11}$ & $\mathrm{U}^{22}$ & $\mathrm{U}^{33}$ & $\mathrm{U}^{23}$ & $\mathrm{U}^{13}$ & $\mathrm{U}^{12}$ \\
\hline $\mathrm{Cu}(1)$ & $51(1)$ & $69(1)$ & $23(1)$ & 0 & 0 & $12(1)$ \\
\hline $\mathrm{P}(1)$ & $63(1)$ & $63(1)$ & $57(2)$ & 0 & 0 & 0 \\
\hline $\mathrm{F}(11)$ & 193(7) & $121(5)$ & $45(3)$ & 0 & 0 & $41(5)$ \\
\hline $\mathrm{F}(12)$ & $105(3)$ & $81(2)$ & $110(3)$ & $-14(2)$ & $21(2)$ & $-24(2)$ \\
\hline $\mathrm{P}(2)$ & $57(1)$ & $57(1)$ & $62(1)$ & 0 & 0 & 0 \\
\hline $\mathrm{F}(21)$ & $125(4)$ & $83(3)$ & $356(10)$ & $31(6)$ & $-4(7)$ & $13(3)$ \\
\hline $\mathrm{F}(22)$ & $169(13)$ & $310(20)$ & $69(5)$ & $45(11)$ & $-41(6)$ & $57(16)$ \\
\hline $\mathrm{N}(11)$ & $48(2)$ & $69(3)$ & $32(2)$ & $-4(2)$ & $-7(2)$ & $11(2)$ \\
\hline $\mathrm{O}(10)$ & $81(2)$ & $65(2)$ & $46(2)$ & $1(2)$ & $-13(2)$ & $-8(2)$ \\
\hline $\mathrm{O}(16)$ & $47(2)$ & $125(4)$ & $60(2)$ & $-24(2)$ & $-11(2)$ & $22(2)$ \\
\hline $\mathrm{C}(11)$ & $41(3)$ & $94(4)$ & $35(2)$ & $-20(3)$ & $-2(2)$ & $6(3)$ \\
\hline $\mathrm{C}(12)$ & $49(3)$ & $89(4)$ & $34(2)$ & $-14(2)$ & $-3(2)$ & $0(3)$ \\
\hline $\mathrm{C}(13)$ & $45(2)$ & $61(3)$ & $28(2)$ & $-11(2)$ & $0(2)$ & $15(2)$ \\
\hline $\mathrm{C}(14)$ & $49(3)$ & $56(3)$ & $38(2)$ & $-9(2)$ & $-5(2)$ & $6(2)$ \\
\hline$C(15)$ & $43(3)$ & $64(3)$ & $37(2)$ & $-5(2)$ & $-3(2)$ & $7(2)$ \\
\hline$C(16)$ & $49(3)$ & $67(3)$ & $36(2)$ & $-11(2)$ & $-3(2)$ & $14(2)$ \\
\hline $\mathrm{N}(21)$ & $41(2)$ & $49(2)$ & $40(2)$ & $-3(2)$ & $4(2)$ & $12(2)$ \\
\hline $\mathrm{O}(26)$ & $75(2)$ & $61(2)$ & $45(2)$ & $8(2)$ & $15(2)$ & $22(2)$ \\
\hline$C(21)$ & $64(3)$ & $69(3)$ & $73(3)$ & $17(3)$ & $29(3)$ & $6(3)$ \\
\hline$C(22)$ & $79(4)$ & $36(3)$ & $71(3)$ & $-6(2)$ & $34(3)$ & $5(2)$ \\
\hline $\mathrm{C}(23)$ & $53(3)$ & $47(3)$ & $36(2)$ & $3(2)$ & $1(2)$ & $0(2)$ \\
\hline$C(24)$ & $71(3)$ & $64(3)$ & $41(3)$ & $-12(2)$ & $20(2)$ & $-12(3)$ \\
\hline$C(25)$ & $93(4)$ & $44(3)$ & $48(3)$ & $-5(2)$ & $22(3)$ & $-1(3)$ \\
\hline$C(26)$ & $48(3)$ & $65(3)$ & $32(2)$ & $3(2)$ & $2(2)$ & $17(2)$ \\
\hline $\mathrm{N}(31)$ & $56(3)$ & $96(4)$ & $41(2)$ & $-21(2)$ & $-1(2)$ & $14(3)$ \\
\hline $\mathrm{N}(32)$ & $73(3)$ & $43(2)$ & $33(2)$ & $2(2)$ & $18(2)$ & $1(2)$ \\
\hline
\end{tabular}




\begin{tabular}{lllllll}
$\mathrm{C}(31)$ & $61(3)$ & $145(6)$ & $44(3)$ & $-21(4)$ & $8(3)$ & $10(3)$ \\
$\mathrm{C}(32)$ & $53(3)$ & $105(5)$ & $41(3)$ & $-15(3)$ & $9(2)$ & $4(3)$ \\
$\mathrm{C}(33)$ & $72(4)$ & $78(4)$ & $40(3)$ & $-6(3)$ & $13(2)$ & $14(3)$ \\
$\mathrm{C}(34)$ & $66(3)$ & $77(4)$ & $41(3)$ & $-3(3)$ & $11(2)$ & $5(3)$ \\
\hline
\end{tabular}

Table 5. Hydrogen coordinates ( x 104) and isotropic displacement parameters $\left(\AA^{2} \times 10^{3}\right)$ for 4 .

\begin{tabular}{|c|c|c|c|c|}
\hline & $\mathrm{x}$ & $\mathrm{y}$ & $\mathrm{z}$ & $\mathrm{U}(\mathrm{eq})$ \\
\hline $\mathrm{H}(11)$ & -116 & 8733 & 11417 & 68 \\
\hline $\mathrm{H}(12)$ & 966 & 8142 & 10894 & 69 \\
\hline $\mathrm{H}(14)$ & 3487 & 9262 & 11522 & 57 \\
\hline $\mathrm{H}(15)$ & 2313 & 9784 & 12039 & 58 \\
\hline $\mathrm{H}(21)$ & 4486 & 7075 & 7607 & 82 \\
\hline $\mathrm{H}(22)$ & 3485 & 7798 & 8137 & 75 \\
\hline $\mathrm{H}(24)$ & 2344 & 4899 & 8374 & 71 \\
\hline $\mathrm{H}(25)$ & 3402 & 4255 & 7850 & 74 \\
\hline $\mathrm{H}(31)$ & 2060 & 8469 & 10383 & 77 \\
\hline $\mathrm{H}(32)$ & 2049 & 5669 & 8974 & 60 \\
\hline $\mathrm{H}(31 \mathrm{~A})$ & 3876 & 8534 & 9977 & 100 \\
\hline $\mathrm{H}(31 \mathrm{~B})$ & 3774 & 7328 & 10109 & 100 \\
\hline $\mathrm{H}(32 \mathrm{~A})$ & 3135 & 7622 & 9438 & 80 \\
\hline $\mathrm{H}(32 \mathrm{~B})$ & 2286 & 8418 & 9616 & 80 \\
\hline $\mathrm{H}(33 \mathrm{~A})$ & 1426 & 7017 & 9949 & 76 \\
\hline $\mathrm{H}(33 \mathrm{~B})$ & 2277 & 6219 & 9769 & 76 \\
\hline $\mathrm{H}(34 \mathrm{~A})$ & 648 & 6198 & 9389 & 74 \\
\hline $\mathrm{H}(34 \mathrm{~B})$ & 869 & 7383 & 9250 & 74 \\
\hline
\end{tabular}

